\title{
New Insights into Secondary Organic Aerosol Formation: Water Binding to Limonene
}

S. Indira Murugachandran, Jackson Tang, Isabel Peñat, Donatella Loruł, M. Eugenia Sanz*

Department of Chemistry, King's College London, London SE1 1DB, UK

\section{Supplementary Information}

[†] Present Address: Departamento de Química Física y Química Inorgánica, Universidad de Valladolid, E-47011 Valladolid, Spain.

[\$] Present Address: Deutsches Elektronen-Synchrotron (DESY), Notkestraße 85, Geb. 25f / Office 354, D-22607 Hamburg.

\section{AUTHOR INFORMATION}

\section{Corresponding Author}

*E-mail: maria.sanz@kcl.ac.uk 


\section{Table of Contents}

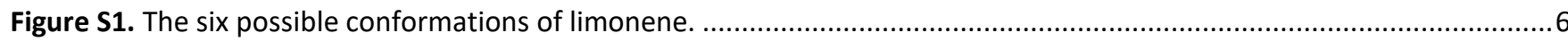

Figure S2. Possible interactions of water with limonene and nomenclature...................................................................

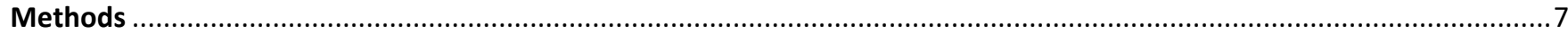

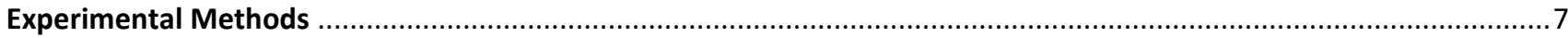

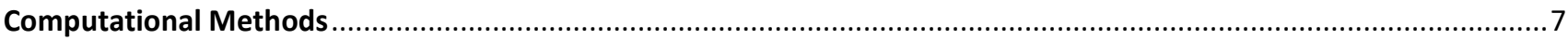

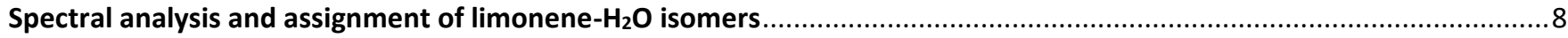

Table S1. Calculated spectroscopic parameters for the isomers of limonene- $\mathrm{H}_{2} \mathrm{O}$ at MP2/6-311++G(d,p) and B3LYP-D3BJ/6$311++G(d, p)$ levels of theory.

.12

Table S1 (cont.). Calculated spectroscopic parameters for the isomers of limonene- $\left(\mathrm{H}_{2} \mathrm{O}\right)$ at MP2/6-311++G(d,p) and B3LYP-

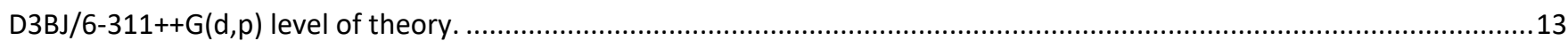

Table S2. Experimental spectroscopic parameters of the observed isotopic species of isomer 1, EQA-3..............................14

Table S3. Experimental spectroscopic parameters of the observed isotopic species of isomer 2, EQA-4............................14

Table S4. Experimental spectroscopic parameters of the observed isotopic species of isomer 3, EQC-4 …........................14

Table S5. Experimental spectroscopic parameters of the observed isotopic species of isomer 4, EQC-2 ............................14

Table S6. Experimental spectroscopic parameters of the observed isotopic species of isomer 6, EQC-3 $\beta \ldots \ldots \ldots \ldots \ldots \ldots \ldots \ldots \ldots \ldots . . .15$

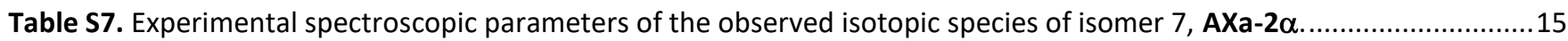

Table S8. Experimental substitution coordinates of the hydrogen and oxygen atoms of water in limonene- $\mathrm{H}_{2} \mathrm{O}$ complexes and the corresponding equilibrium coordinates from MP2 and B3LYP-D3BJ calculations using the $6-311++G(d, p)$ basis set........16

Table $\mathbf{8 8}$ (cont.). Experimental substitution coordinates of the hydrogen and oxygen atoms of water in limonene- $\mathrm{H}_{2} \mathrm{O}$ complexes compared with equilibrium coordinates from MP2 and B3LYP-D3BJ calculations using the 6-311++G(d,p) basis set.

.17

Table 59. Intermolecular stabilising energy contributions $\left(\geq 0.42 \mathrm{~kJ} \mathrm{~mol}^{-1}\right)$ for isomer EQA-3 of limonene- $\mathrm{H}_{2} \mathrm{O}$ from Natural Bond Orbital (NBO) analysis at the B3LYP-D3BJ/6-311++G(d,p) level of theory. .18

Table S10. Intermolecular stabilising energy contributions $\left(\geq 0.42 \mathrm{~kJ} \mathrm{~mol}^{-1}\right)$ for isomer EQA-4 of limonene- $\mathrm{H}_{2} \mathrm{O}$ from Natural Bond Orbital (NBO) analysis at the B3LYP-D3BJ/6-311++G(d,p) level of theory.

Table S11. Intermolecular stabilising energy contributions $\left(\geq 0.42 \mathrm{~kJ} \mathrm{~mol}^{-1}\right)$ for isomer EQC-3 $\beta$ of limonene- $\mathrm{H}_{2} \mathrm{O}$ from Natural Bond Orbital (NBO) analysis at the B3LYP-D3BJ/6-311++G(d,p) level of theory. . .18

Table S12. Intermolecular stabilising energy contributions $\left(\geq 0.42 \mathrm{~kJ} \mathrm{~mol}^{-1}\right)$ for isomer EQC-4 of limonene- $\mathrm{H}_{2} \mathrm{O}$ from Natural Bond Orbital (NBO) analysis at the B3LYP-D3BJ/6-311++G(d,p) level of theory. .19

Table S13. Intermolecular stabilising energy contributions $\left(\geq 0.42 \mathrm{~kJ} \mathrm{~mol}^{-1}\right)$ for isomer EQC-2 of limonene- $\mathrm{H}_{2} \mathrm{O}$ from Natural Bond Orbital (NBO) analysis at the B3LYP-D3BJ/6-311++G(d,p) level of theory.

Table S14. Intermolecular stabilising energy contributions $\left(\geq 0.42 \mathrm{~kJ} \mathrm{~mol}^{-1}\right)$ for isomer EQa-4 of limonene- $\mathrm{H}_{2} \mathrm{O}$ from Natural Bond Orbital (NBO) analysis at the B3LYP-D3BJ/6-311++G(d,p) level of theory. .19

Table S15. Intermolecular stabilising energy contributions $\left(\geq 0.42 \mathrm{~kJ} \mathrm{~mol}^{-1}\right)$ for isomer $\mathbf{A X a - 2} \alpha$ of limonene- $\mathrm{H}_{2} \mathrm{O}$ from Natural Bond Orbital (NBO) analysis at the B3LYP-D3BJ/6-311++G(d,p) level of theory. 
Table S16. Energy decomposition (SAPT2(+3) $\delta_{\mathrm{MP} 2} /$ aug-cc-pDVZ) in $\mathrm{kJmol}^{-1}$ for the observed isomers of limonene- $\mathrm{H}_{2} \mathrm{O}$ on

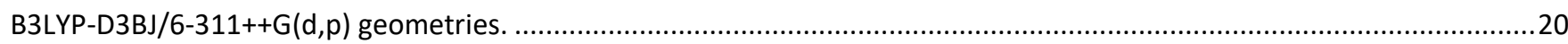

Table S17. Cartesian coordinates of isomer EQA-3 from B3LYP-D3BJ/6-311++G(d,p) calculations. ................................21

Table S18. Cartesian coordinates of isomer EQA-4 from B3LYP-D3BJ/6-311++G(d,p) calculations. ................................22

Table S19. Cartesian coordinates of isomer EQC-4 from B3LYP-D3BJ/6-311++G(d,p) calculations..................................22

Table S20. Cartesian coordinates of isomer EQC-2 from B3LYP-D3BJ/6-311++G(d,p) calculations.................................23

Table S21. Cartesian coordinates of isomer EQa-4 from B3LYP-D3BJ/6-311++G(d,p) calculations................................23

Table S22. Cartesian coordinates of isomer EQC-3 $\beta$ from B3LYP-D3BJ/6-311++G(d,p) calculations. ..............................24

Table S23. Cartesian coordinates of isomer AXa-2 $\alpha$ from B3LYP-D3BJ/6-311++G(d,p) calculations. ..............................24

Figure S3. Interconversion barrier between isomers EQC-3 $\boldsymbol{\alpha}$ and EQC-3 $\beta$ of limonene- $\mathrm{H}_{2} \mathrm{O}$ at the B3LYP-D3BJ/6-.............25

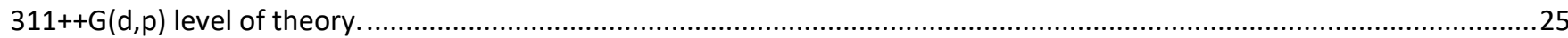

Figure S4. Interconversion barrier between isomers EQA-2 and EQC-2 of limonene- $\mathrm{H}_{2} \mathrm{O}$ at the B3LYP-D3BJ/6-311++G(d,p)

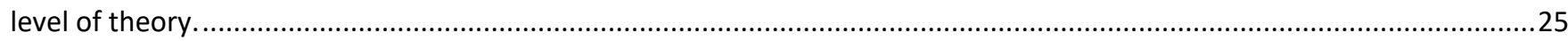

Figure S5. Interconversion barriers between isomers EQA-3, EQC-3 and EQa-3 of limonene- $\mathrm{H}_{2} \mathrm{O}$ at the B3LYP-D3BJ/6-

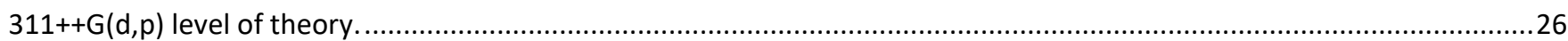

Figure S6. Interconversion barriers between isomers EQA-4, EQC-4 and EQa-4 of limonene- $\mathrm{H}_{2} \mathrm{O}$ at the B3LYP-D3BJ/6-

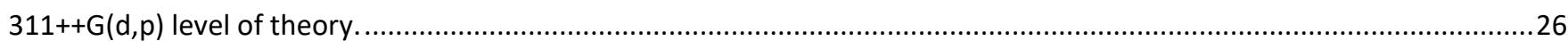

Figure S7. Interconversion barriers between isomers AXa-4 and EQa-4 of limonene- $\mathrm{H}_{2} \mathrm{O}$ at the B3LYP-D3BJ/6-311++G(d,p)

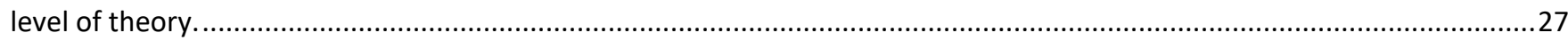

Figure S8. Interconversion barriers between isomers AXa-3 and EQC-3 of limonene- $\mathrm{H}_{2} \mathrm{O}$ at the B3LYP-D3BJ/6-311++G(d,p) level of theory.......

.27

Figure S9. Interconversion barriers between isomers $\mathbf{A X a - 3 ,} \mathbf{A X a - 2} \boldsymbol{\alpha}$ and $\mathbf{A X a - 2} \boldsymbol{\beta}$ of limonene- $\mathrm{H}_{2} \mathrm{O}$ at the $\mathbf{M P 2} / 6-311++\mathrm{G}(\mathrm{d}, \mathrm{p})$ level of theory.

Figure S10. Interconversion barriers between isomers AXa-3, AXa-2 $\alpha$ and AXa-2 $\boldsymbol{\beta}$ of limonene- ${ }_{2} \mathrm{O}$ at the B3LYP-D3BJ/6$311++G(d, p)$ level of theory. 28

Figure S11. 3D plots of the molecular electrostatic potential (MEP) mapped on the $0.001 E_{h}$ isodensity surface calculated at the MP2/6-311++G(d,p) level of theory for the observed conformers of limonene. Areas of high electronegativity have been labelled with their values in $\mathrm{kJmol}^{-1}$. For the exocyclic double bond where one side of the surface is visible, the first value given corresponds to the visible surface and the second for the surface out of view..... 29

Figure S12. Plots of the reduced density gradient (RDG) versus $\operatorname{sign}(\lambda 2) \rho$ for the observed isomers of limonene- $\mathrm{H}_{2} \mathrm{O} \ldots \ldots . . . . .30$

Frequencies of measured transitions and their residuals.

32

Table S24. Measured frequencies ( $\left.v_{\text {obs }}\right)$ and residuals ( $\left.v_{\text {obs }}-v_{\text {cal }}\right)$ of the rotational transitions of the parent species of isomer EQA-3 of limonene- $\mathrm{H}_{2} \mathrm{O}$.

Table S25. Measured frequencies ( $\left.v_{\text {obs }}\right)$ and residuals ( $v_{\text {obs }}-v_{\text {cal }}$ ) of the rotational transitions of the DOH isotopic species of isomer EQA-3 of limonene- $\mathrm{H}_{2} \mathrm{O}$

Table S26. Measured frequencies ( $v_{\text {obs }}$ ) and residuals ( $v_{\text {obs }}-v_{\text {cal }}$ ) of the rotational transitions of the HOD isotopic species of isomer EQA-3 of limonene- $\mathrm{H}_{2} \mathrm{O}$ 
Table S27. Measured frequencies ( $v_{\text {obs }}$ ) and residuals ( $v_{\text {obs }}-v_{\text {cal }}$ ) of the rotational transitions of the DOD isotopic species of isomer EQA-3 of limonene- $\mathrm{H}_{2} \mathrm{O}$.

Table S28. Measured frequencies ( $\left.v_{\text {obs }}\right)$ and residuals ( $v_{\text {obs }}-v_{\text {cal }}$ ) of the rotational transitions of the $\mathrm{H}_{2}{ }^{18} \mathrm{O}$ isotopic species of isomer EQA-3 of limonene- $\mathrm{H}_{2} \mathrm{O}$.

Table S29. Measured frequencies ( $\left.v_{\text {obs }}\right)$ and residuals ( $\left.v_{\text {obs }}-v_{\text {cal }}\right)$ of the rotational transitions of the parent species of isomer EQA-4 of limonene- $\mathrm{H}_{2} \mathrm{O}$.

Table S30. Measured frequencies ( $v_{\text {obs }}$ ) and residuals ( $v_{\text {obs }}-v_{\text {cal }}$ ) of the rotational transitions of the DOH isotopic species of isomer EQA-4 of limonene- $\mathrm{H}_{2} \mathrm{O}$

Table S31. Measured frequencies ( $\left.v_{\text {obs }}\right)$ and residuals ( $v_{\text {obs }}-v_{\text {cal }}$ ) of the rotational transitions of the HOD isotopic species of isomer EQA-4 of limonene- $\mathrm{H}_{2} \mathrm{O}$

Table S32. Measured frequencies ( $\left.v_{\text {obs }}\right)$ and residuals $\left(v_{\text {obs }}-v_{\text {cal }}\right)$ of the rotational transitions of the DOD isotopic species of isomer EQA-4 of limonene- $\mathrm{H}_{2} \mathrm{O}$ .39

Table S33. Measured frequencies ( $v_{\text {obs }}$ ) and residuals ( $v_{\text {obs }}-v_{\text {cal }}$ ) of the rotational transitions of the $\mathrm{H}_{2}{ }^{18} \mathrm{O}$ isotopic species of isomer EQA-4 of limonene- $\mathrm{H}_{2} \mathrm{O}$.

Table S34. Measured frequencies ( $v_{\text {obs }}$ ) and residuals ( $v_{\text {obs }}-v_{\text {cal }}$ ) of the rotational transitions of the parent species of isomer EQC-4 of limonene- $\mathrm{H}_{2} \mathrm{O}$.

Table S35. Measured frequencies ( $\left.v_{\text {obs }}\right)$ and residuals $\left(v_{\text {obs }}-v_{\text {cal }}\right.$ ) of the rotational transitions of the DOH isotopic species of isomer EQC-4 of limonene- $\mathrm{H}_{2} \mathrm{O}$.

Table S36. Measured frequencies ( $\left.v_{\text {obs }}\right)$ and residuals ( $v_{\text {obs }}-v_{\text {cal }}$ ) of the rotational transitions of the HOD isotopic species of isomer EQC-4 of limonene- $\mathrm{H}_{2} \mathrm{O}$.

Table S37. Measured frequencies ( $v_{\text {obs }}$ ) and residuals ( $v_{\text {obs }}-v_{\text {cal }}$ ) of the rotational transitions of the DOD isotopic species of isomer EQC-4 of limonene- $\mathrm{H}_{2} \mathrm{O}$.

Table S38. Measured frequencies ( $v_{\mathrm{obs}}$ ) and residuals ( $\left.v_{\mathrm{obs}}-v_{\mathrm{cal}}\right)$ of the rotational transitions of the $\mathrm{H}_{2}{ }^{18} \mathrm{O}$ isotopic species of isomer EQC-4 of limonene- $\mathrm{H}_{2} \mathrm{O}$.

Table S39. Measured frequencies ( $\left.v_{\text {obs }}\right)$ and residuals ( $\left.v_{\text {obs }}-v_{\text {cal }}\right)$ of the rotational transitions of the parent species of isomer EQC-2 of limonene- $\mathrm{H}_{2} \mathrm{O}$.

Table S40. Measured frequencies ( $\left.v_{\text {obs }}\right)$ and residuals $\left(v_{\text {obs }}-v_{\text {cal }}\right.$ ) of the rotational transitions of the DOH isotopic species of isomer EQC-2 of limonene- $\mathrm{H}_{2} \mathrm{O}$. .46

Table S41. Measured frequencies ( $\left.v_{\text {obs }}\right)$ and residuals ( $v_{\text {obs }}-v_{\text {cal }}$ ) of the rotational transitions of the HOD isotopic species of isomer EQC-2 of limonene- $\mathrm{H}_{2} \mathrm{O}$.

Table S42. Measured frequencies ( $v_{\text {obs }}$ ) and residuals ( $v_{\text {obs }}-v_{\text {cal }}$ ) of the rotational transitions of the DOD isotopic species of isomer EQC-2 of limonene- $\mathrm{H}_{2} \mathrm{O}$.

Table S43. Measured frequencies ( $v_{\text {obs }}$ ) and residuals ( $\left.v_{\text {obs }}-v_{\text {cal }}\right)$ of the rotational transitions of the $\mathrm{H}_{2}{ }^{18} \mathrm{O}$ isotopic species of isomer EQC-2 of limonene- $\mathrm{H}_{2} \mathrm{O}$.

Table S44. Measured frequencies ( $\left.v_{\text {obs }}\right)$ and residuals $\left(v_{\text {obs }}-v_{\text {cal }}\right)$ of the rotational transitions of the parent species of isomer EQa-4 of limonene- $\mathrm{H}_{2} \mathrm{O}$.

Table S45. Measured frequencies ( $v_{\text {obs }}$ ) and residuals ( $v_{\text {obs }}-v_{\text {cal }}$ ) of the rotational transitions of the parent species of isomer EQC-3 $\beta$ of limonene- $\mathrm{H}_{2} \mathrm{O}$ 
Table S46. Measured frequencies ( $v_{\text {obs }}$ ) and residuals ( $v_{\text {obs }}-v_{\text {cal }}$ ) of the rotational transitions of the DOH isotopic species of isomer EQC-3 $\beta$ of limonene- $\mathrm{H}_{2} \mathrm{O}$.

Table S47. Measured frequencies ( $\left.v_{\text {obs }}\right)$ and residuals ( $v_{\text {obs }}-v_{\text {cal }}$ ) of the rotational transitions of the HOD isotopic species of isomer EQC-3 $\beta$ of limonene- $\mathrm{H}_{2} \mathrm{O}$.

Table S48. Measured frequencies ( $v_{\text {obs }}$ ) and residuals ( $v_{\text {obs }}-v_{\text {cal }}$ ) of the rotational transitions of the DOD isotopic species of isomer EQC-3 $\beta$ of limonene- $\mathrm{H}_{2} \mathrm{O}$. .50

Table S49. Measured frequencies ( $v_{\mathrm{obs}}$ ) and residuals ( $v_{\mathrm{obs}}-v_{\mathrm{cal}}$ ) of the rotational transitions of the $\mathrm{H}_{2}{ }^{18} \mathrm{O}$ isotopic species of isomer EQC-3 $\beta$ of limonene- $\mathrm{H}_{2} \mathrm{O}$. .

Table S50. Measured frequencies (vobs) and residuals ( $v_{\text {obs }}-v_{\text {cal }}$ ) of the rotational transitions of the parent species of isomer $\mathbf{A X a - 2} \alpha$ of limonene- $\mathrm{H}_{2} \mathrm{O}$.

Table S51. Measured frequencies ( $v_{\text {obs }}$ ) and residuals ( $v_{\text {obs }}-v_{\text {cal }}$ ) of the rotational transitions of the DOH isotopic species of isomer $\mathbf{A X a - 2} \boldsymbol{\alpha}$ of limonene- $\mathrm{H}_{2} \mathrm{O}$.

Table S52. Measured frequencies ( $\left.v_{\text {obs }}\right)$ and residuals ( $v_{\text {obs }}-v_{\text {cal }}$ ) of the rotational transitions of the DOD isotopic species of isomer $\mathrm{AXa-2} \alpha$ of limonene- $\mathrm{H}_{2} \mathrm{O}$. .52

Table S53. Measured frequencies ( $v_{\text {obs }}$ ) and residuals ( $v_{\text {obs }}-v_{\text {cal }}$ ) of the rotational transitions of the $\mathrm{H}_{2}{ }^{18} \mathrm{O}$ isotopic species of isomer $\mathbf{A X a - 2} \boldsymbol{\alpha}$ of limonene- $\mathrm{H}_{2} \mathrm{O}$. 
Figure S1. The six possible conformations of limonene.

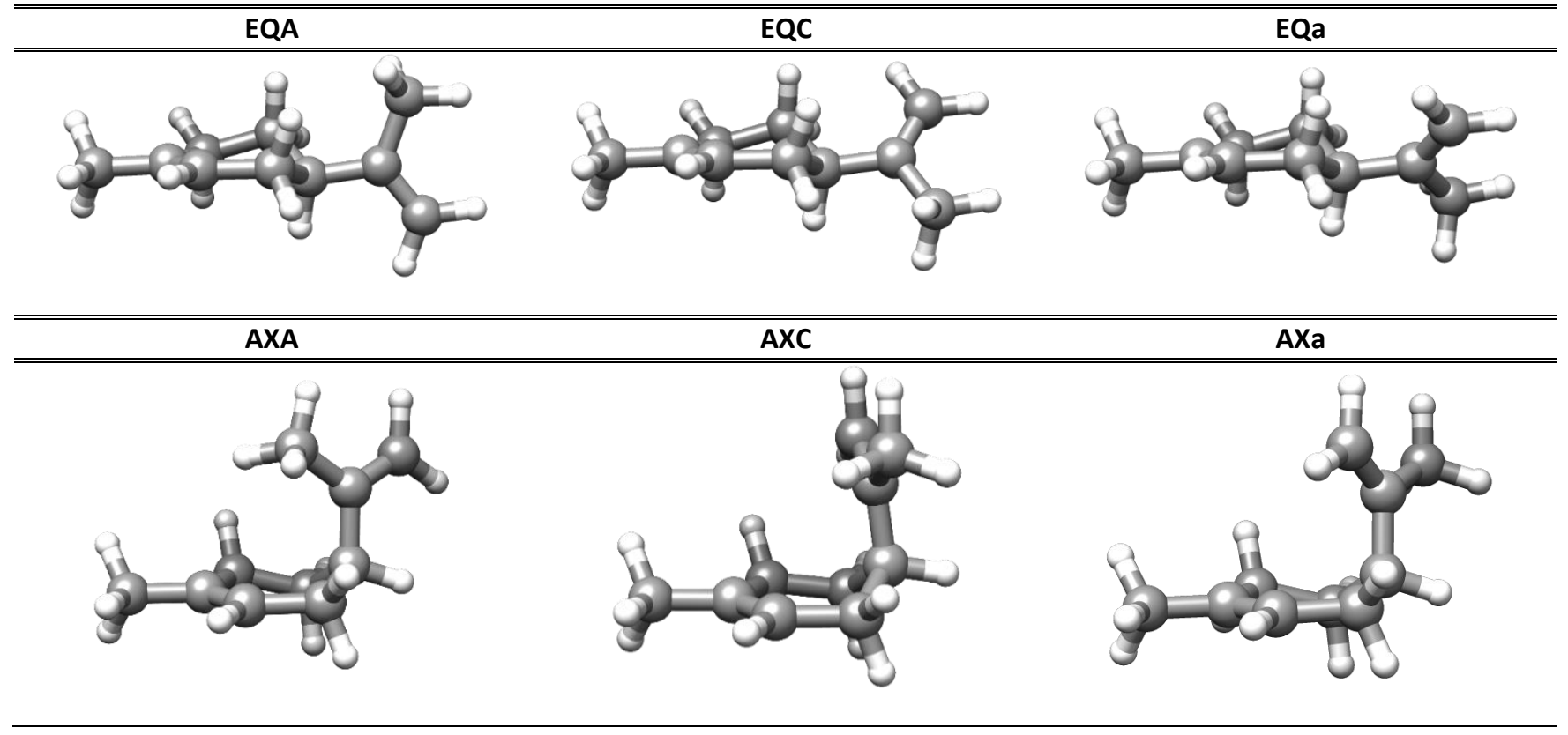

Figure S2. Possible interactions of water with limonene and nomenclature.

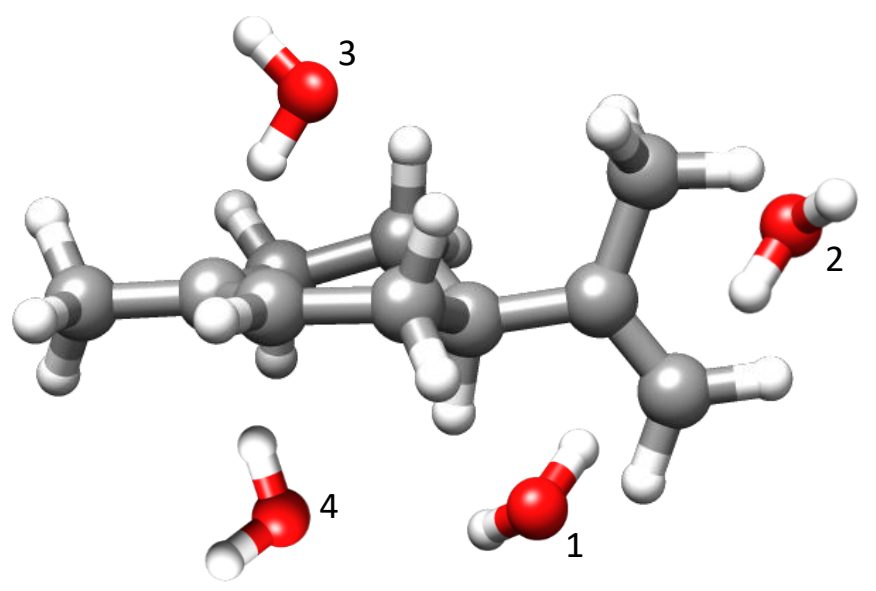




\section{Methods}

\section{Experimental Methods}

The limonene-water rotational spectrum was collected in the $2-8 \mathrm{GHz}$ range using the chirped-pulse Fourier transform microwave (CP-FTMW) spectrometer at King's College London ${ }^{1,2}$. Complexes of limonene-water are formed in the supersonic expansion of a mixture of $(R)-(+)$-limonene $(97 \%$, Sigma-Aldrich) and water seeded in neon into the vacuum chamber. The complexes interact with four chirped microwave pulses of $4 \mu \mathrm{s}$ length, varying linearly in the $2-8 \mathrm{GHz}$ frequency range and with a $30 \mu$ s delay between them, that are broadcast into the chamber via a broadband microwave horn. Molecular emission signals are collected for $20 \mu \mathrm{s}$ after each microwave excitation pulse by a second microwave horn, amplified, and stored in the time domain on a high speed oscilloscope. A fast Fourier transform algorithm is applied to the collected free induction decays (FIDs) to convert the time domain spectrum into the frequency domain.

Optimal line intensity was obtained by flowing Ne at 6 bar through water, placed in a reservoir in the injection line, and limonene. The latter was located in a bespoke receptacle attached to the nozzle, where it was gently heated to $\mathrm{ca}$. $340 \mathrm{~K}$. The final spectrum has $1 \mathrm{M}$ FIDs. Experiments performed to observe the ${ }^{18} \mathrm{O}$ isotopologues of limonene-water complexes were carried out with a $1: 1$ mixture of $\mathrm{H}_{2}{ }^{16} \mathrm{O}: \mathrm{H}_{2}{ }^{18} \mathrm{O}$ (97\% purity, Chem-Cruz). Experiments to observe heavy water isotopologues were performed with a 1:1 mixture of $\mathrm{H}_{2} \mathrm{O}: \mathrm{D}_{2} \mathrm{O}$ and with pure $\mathrm{D}_{2} \mathrm{O}$ (100\% purity, Euriso-Top).

\section{Computational Methods}

Theoretical calculations were carried out to explore the potential energy surface of the limonene- $\mathrm{H}_{2} \mathrm{O}$ complexes and predict the lower-energy configurations, their relative energies, and the molecular properties relevant for the analysis of the rotational spectrum, namely rotational constants and dipole moment components. We considered all six conformations of limonene ${ }^{3,4}$ and different arrangements of the water molecule, taking into account interactions with areas of significant electron density in limonene. These are the double bonds, that can act as hydrogen bond acceptors. We used labels 1 and 2 to refer to interactions of water with the exocyclic double bond of limonene on its opposite sides, and 3 and 4 to refer to interactions with the endocyclic double bond above and below the cyclohexene ring, respectively. Starting structures were optimised using B3LYP-D3BJ (B3LYP functional including Grimme's correction to dispersion and Becke-Johnson damping) $)^{5,6}$ and MP2 methods with the 6$311++G(d, p)$ basis set, using the Gaussian 09 software package. ${ }^{7}$ A total of 30 different isomers with energies up to $16.7 \mathrm{~kJ} \mathrm{~mol}^{-1}$ were obtained. Those within $12 \mathrm{~kJ} \mathrm{~mol}^{-1}$ of the global minimum are shown in Tables 1 and S1. Additional explorations of the potential energy surface were performed with the CREST program ${ }^{8}$ which samples the potential energy surface using a semiempirical method and generates conformational ensembles. Structures from CREST were subsequently optimised using B3LYP-D3BJ and MP2 methods, but no additional isomers were predicted below $12 \mathrm{~kJ} \mathrm{~mol}^{-1}$.

The energies of the predicted complexes were corrected following the counterpoise (CP) procedure $^{9}$, which removes basis set superposition errors (BSSE), and including corrections considering the relaxation of the geometries of each moiety from their bare structures. ${ }^{10}$ 


\section{Spectral analysis and assignment of limonene- $\mathrm{H}_{2} \mathrm{O}$ isomers}

Identifying the observed isomers of limonene- $\mathrm{H}_{2} \mathrm{O}$ is tremendously challenging as most of them have very similar rotational constants. Only isomer 7 has rotational constants markedly different from the others, indicative of limonene being in an axial conformation. We have only observed $a$-type spectrum for this isomer, which shows that the dipole moment component $\mu_{\mathrm{a}}$ is predominant and leads to the assignment of isomer 7 to complex AXa$\mathbf{2} \alpha$. Other isomers with similar rotational constants (see Tables 1 and S1) are predicted to have a sizable $\mu_{\mathrm{b}}$ dipole moment component and thus would also show $b$-type spectrum. The assignment was confirmed with the observation of the $\mathrm{H}_{2}^{18} \mathrm{O}, \mathrm{DOH}$ and $\mathrm{D}_{2} \mathrm{O}$ isotopologues at the predicted frequency shifts (Tables S7, S8). The HOD isotopologue could not be observed for isomer 7. It is probably within the noise level, as it is expected to have a lower intensity than the $\mathrm{DOH}$ isotopologue due to zero-point effects.

Considering the other observed species, isomers 1 and 2 have extremely close rotational constants, and $a$-, $b$ - and $c$-type lines have been observed for both of them. Based on this, and comparing experimental with theoretical rotational constants, these isomers could correspond to complexes EQA-3 and EQA-4. We can discriminate them from the different relative intensities of their rotational transitions. Isomer 1 shows $a$-type lines of much higher intensity than $b$ - or $c$ - type, while all types of lines of isomer 2 show similar intensities. In our experiment, transition intensity is directly proportional to the square of the corresponding dipole moment component. Considering this and the predicted dipole moment components (Table 1), relative intensities should be approximately $\mu_{\mathrm{a}}{ }^{2}: \mu_{\mathrm{b}}{ }^{2}: \mu_{\mathrm{c}}{ }^{2}$ = $5: 1: 1$ for EQA-3 and $1: 1: 1$ for EQA-4. Therefore, we can unambiguously identify isomer 1 as EQA-3 and isomer 2 as EQA-4. Further confirmation was obtained from the observation of their $\mathrm{HOD}, \mathrm{DOH}, \mathrm{H}_{2}{ }^{18} \mathrm{O}$ and $\mathrm{D}_{2} \mathrm{O}$ isotopologues at the predicted frequency shifts (Tables S2 and S3). From the differences between the moments of inertia of the parent and mono-substituted isotopic species, we determined the positions of the substituted atoms in the principal axis system using Kraitchman's method ${ }^{11}$ and the KRA and EVAL programs ${ }^{12}$ (Table S8). It can be seen how close their values are for these two isomers, and their consistency with assignments. This is also manifest in the overlays depicted in Fig. 2.

Isomer 3 can be assigned to complex EQC-4 from the comparison of experimental and calculated rotational constants and the non-observation of $b$-type transitions, which is consistent with the predicted very low value of the $\mu_{\mathrm{b}}$ dipole moment component. Similarly, the non-observation of $a$-type transitions for isomer 6 allows its assignment to EQC-3 $\beta$. Isomers EQC-3 $\beta$ and EQC-3 $\alpha$ (see Fig. S3) have almost the same structure but differ in the orientation of the water molecule, which pivots by about $45^{\circ}$. This causes a drastic variation in $\mu_{\mathrm{a}}$, whose value changes from $c a .1 \mathrm{D}$ in EQC-3 $\alpha$ to zero in EQC-3 $\beta$. The interconversion barrier between these two isomers is very low (ca. $5 \mathrm{~cm}^{-1}$, Fig. S3), which is consistent with the presence of only one of them since it is well known that higher-energy species can relax to lower-energy ones in a supersonic if their interconversion barriers are sufficiently low ${ }^{13,14}$.

Isomer 4 can be matched to isomers EQA-2 or EQC-2. The observation of $a$-, $b$ - and $c$-type spectrum for isomer 4 points to isomer 4 being EQC-2. A definitive assignment of isomer 4 to EQC-2 was achieved from the values of the experimental $r_{s}$ substitution coordinates and their comparison with the computational ones (Fig. 2 and Table S8). The interconversion barrier between EQA-2 and EQC-2 has been calculated at B3LYP-D3BJ/6-311++G(d,p) to be $\sim 1200 \mathrm{~cm}^{-1}$, high enough to prevent the relaxation of EQC-2 to EQA-2 (Fig. S4). 
Isomer 5 can be assigned to complex EQa-4 from the comparison between experimental and rotational constants and the observation of only $a$-type spectrum. Unfortunately, isomer 5 has a weak spectrum and no transitions arising from isotopically-enriched water species could be observed. There are no other predicted isomers with rotational constants similar to those determined for isomer 5 . However, low barrier heights have been calculated for the interconversion of EQa-3 and EQa-4 complexes to EQC-3 and EQC-4, respectively (Figs. S5, S6). This behaviour mimics that observed for bare limonene, and shows that interactions with one water molecule do not significantly influence the interconversion between limonene conformers. For limonene, we found that, despite the low interconversion barrier, EQa was present in our supersonic expansion when Ne was used as a carrier gas ${ }^{3}$. We believe that the same occurs for the complex with one water molecule allowing the observation of EQa-4, albeit with low abundance.

We have looked exhaustively for other isomers predicted to be lower in energy than some of those observed experimentally but we have not found them. Isomer EQa-3 can relax to EQC3 though a low barrier of about 100 $\mathrm{cm}^{-1}$. Isomers EQA-1 and EQA-2 have lower binding energies than other isomers of limonene- $\mathrm{H}_{2} \mathrm{O}$ involving the conformer EQA of limonene and are probably not populated enough in our supersonic jet. We have tried to explore their possible relaxation to EQA-3 and EQA-4, but this involves multi-coordinate pathways and it has not been possible to find an interconversion path using the STQN method in Gaussian. The axial isomers would not relax in a supersonic jet to equatorial isomers, as the interconversion barriers are above $2000 \mathrm{~cm}^{-1}$ (see Figs. S7 and S8). The non-observation of AXa-4 can be attributed to the lower dipole moment than $\mathbf{A X a - 2} \alpha$, that will make its spectrum weaker than that of AXa-2 $\alpha$ (already weak). AXa-3 is lower in energy than AXa-2 $\alpha$ at B3LYP-D3BJ level but higher at MP2 level. Their interconversion barrier at both levels of theory is very low, allowing relaxation in our supersonic jet (see Figs. S9 and S10), but pointing to different isomers as the lowest-energy one. The values are within the energy uncertainties for these calculations, showing how experimental data is vital to determine the species present in the supersonic jet. 


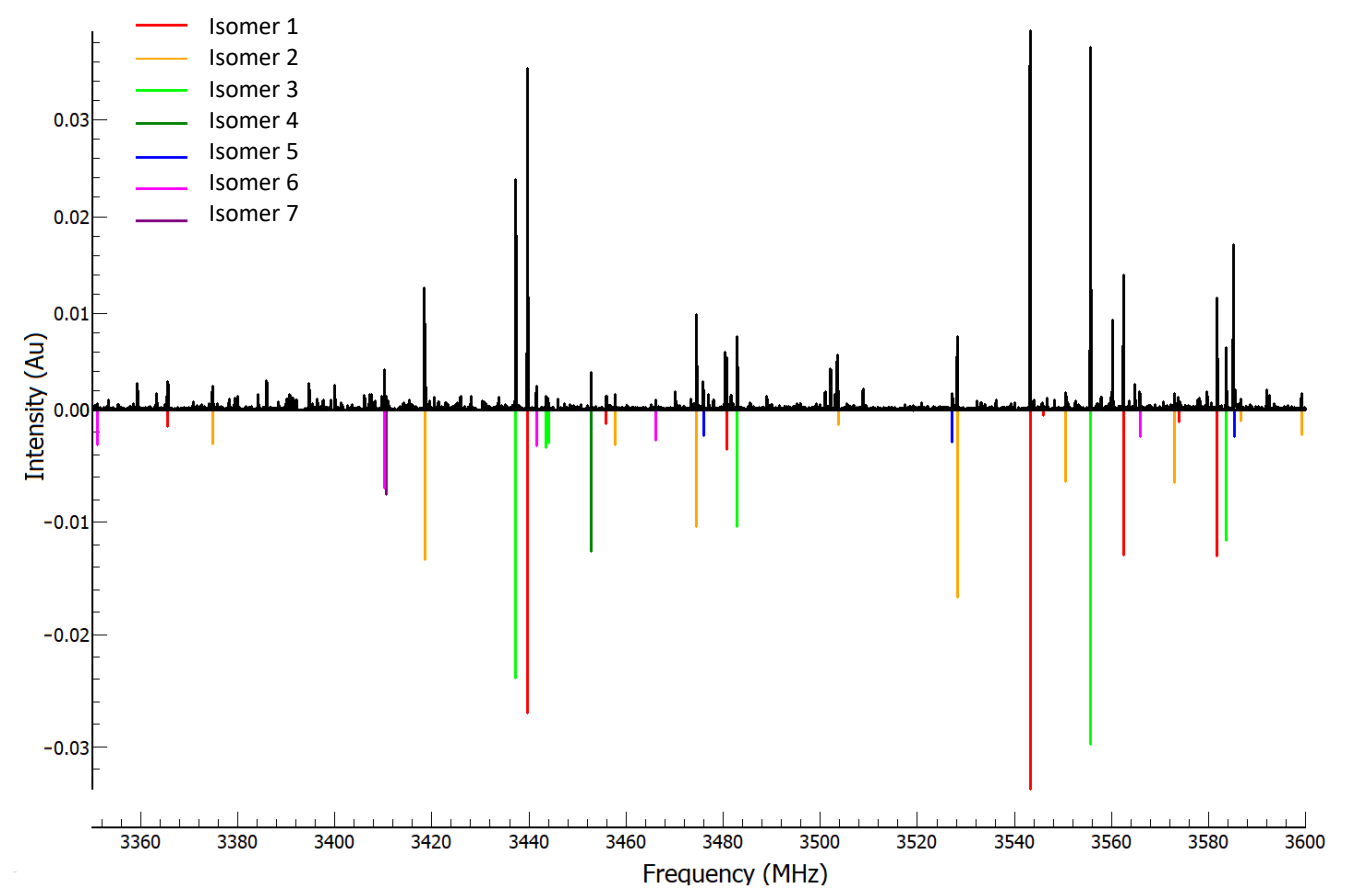

Section of the experimental spectrum of limonene-water between $3350-3600 \mathrm{MHz}$. Upper trace shows the experimental spectrum, lower trace shows the simulated spectrum simulated using the experimentally determined rotational constants.

(1) Loru, D.; Bermúdez, M. A.; Sanz, M. E. Structure of Fenchone by Broadband Rotational Spectroscopy Structure of Fenchone by Broadband Rotational Spectroscopy. J. Chem. Phys. 2016, 145, 074311-074318. https://doi.org/10.1063/1.4961018.

(2) Loru, D.; Peña, I.; Sanz, M. E. Ethanol Dimer: Observation of Three New Conformers by Broadband Rotational Spectroscopy. J. Mol. Spectrosc. 2017, 335, 93-101. https://doi.org/10.1016/j.jms.2017.03.007.

(3) Loru, D.; Vigorito, A.; Santos, A. F. M.; Tang, J.; Sanz, M. E. The Axial/Equatorial Conformational Landscape and Intramolecular Dispersion: New Insights from the Rotational Spectra of Monoterpenoids. Phys. Chem. Chem. Phys. 2019, 21, 26111-26116. https://doi.org/10.1039/c9cp05264j.

(4) Moreno, J. R. A.; Huet, T. R.; González, J. J. L. Conformational Relaxation of S-(+)-Carvone and R-(+)-Limonene Studied by Microwave Fourier Transform Spectroscopy and Quantum Chemical Calculations. Struct. Chem. 2013, 24 (4), 1163-1170. https://doi.org/10.1007/s11224-012-0142-8.

(5) Grimme, S.; Ehrlich, S.; Goerigk, L. Effect of the Damping Function in Dispersion Corrected Density Functional Theory. J. Comput. Chem. 2011, 32, 1456-1465. https://doi.org/10.1002/jcc.21759.

(6) Grimme, S.; Antony, J.; Ehrlich, S.; Krieg, H. A Consistent and Accurate Ab Initio Parametrization of Density Functional Dispersion Correction (DFT-D) for the 94 Elements H-Pu. J. Chem. Phys. 2010, 132, 154104. https://doi.org/10.1063/1.3382344.

(7) Frisch, M. J.; Trucks, G. W.; Schlegel, H. B.; Scuseria, G. E.; Robb, M. A.; Cheeseman, J. R.; Scalmani, G.; Barone, V.; Mennucci, B.; Petersson, G. A.; Nakatsuji, H.; Caricato, M.; Li, X.; Hratchian, H. P.; Izmaylov, A. F.; Bloino, J.; Zheng, G.; Sonnenberg, J. L.; Hada, M.; Ehara, M.; Toyota, K.; Fukuda, R.; Hasegawa, J.; Ishida, M.; Nakajima, T.; Honda, Y.; Kitao, O.; Nakai, H.; Vreven, T.; Montgomery, J. A., J.; Peralta, J. E.; Ogliaro, F.; Bearpark, M.; Heyd, J. J.; Brothers, E.; 
Kudin, K. N.; Staroverov, V. N.; Kobayashi, R.; Normand, J.; Raghavachari, K.; Rendell, A.; Burant, J. C.; Iyengar, S. S.; Tomasi, J.; Cossi, M.; Rega, N.; Millam, J. M.; Klene, M.; Knox, J. E.; Cross, J. B.; Bakken, V.; Adamo, C.; Jaramillo, J.; Gomperts, R.; Stratmann, R. E.; Yazyev, O.; Austin, A. J.; Cammi, R.; Pomelli, C.; Ochterski, J. W.; Martin, R. L.; Morokuma, K.; Zakrzewski, V. G.; Voth, G. A.; Salvador, P.; Dannenberg, J. J.; Dapprich, S.; Daniels, A. D.; Farkas, Ö.; Foresman, J. B.; Ortiz, J. V.; Cioslowski, J.; Fox, D. J. Gaussian 09, Revision E. 01; Gaussian; 2009. https://doi.org/111.

(8) Pracht, P.; Bohle, F.; Grimme, S. Automated Exploration of the Low-Energy Chemical Space with Fast Quantum Chemical Methods. Phys. Chem. Chem. Phys. 2020, 22 (14), 7169-7192. https://doi.org/10.1039/c9cp06869d.

(9) Boys, S. F.; Bernardi, F. The Calculation of Small Molecular Interactions by the Differences of Separate Total Energies. Some Procedures with Reduced Errors. Mol. Phys. 1970, 19 (4), 553-566. https://doi.org/10.1080/00268977000101561.

(10) Xantheas, S. S. On the Importance of the Fragment Relaxation Energy Terms in the Estimation of the Basis Set Superposition Error Correction to the Intermolecular Interaction Energy. J. Chem. Phys. 1996, 104 (21), 8821-8824. https://doi.org/10.1063/1.471605.

(11) Kraitchman, J. Determination of Molecular Structure from Microwave Spectroscopic Data. Am. J. Phys. 1953, 21 (1), 17-24. https://doi.org/10.1119/1.1933338.

(12) Kisiel, Z. PROSPE - Programs for ROtational SPEctroscopy. Spectrosc. from Sp. 2001, 91-106.

(13) Ruoff, R. S.; Klots, T. D.; Emilsson, T.; Gutowsky, H. S. Relaxation of Conformers and Isomers in Seeded Supersonic Jets of Inert Gases. J. Chem. Phys. 1990, 93 (5), 3142-3150. https://doi.org/10.1063/1.458848.

(14) Erlekam, U.; Frankowski, M.; Von Helden, G.; Meijer, G. Cold Collisions Catalyse Conformational Conversion. Phys. Chem. Chem. Phys. 2007, 9, 3786-3789. https://doi.org/10.1039/b703571c. 
Table S1. Calculated spectroscopic parameters for the isomers of limonene $-\mathrm{H}_{2} \mathrm{O}$ at MP2/6-311++G(d,p) and B3LYP$\mathrm{D} 3 \mathrm{BJ} / 6-311++\mathrm{G}(\mathrm{d}, \mathrm{p})$ levels of theory.

\begin{tabular}{|c|c|c|c|c|c|c|c|c|c|c|c|c|}
\hline & \multicolumn{2}{|c|}{ EQA-3 } & \multicolumn{2}{|c|}{ EQA-4 } & \multicolumn{2}{|c|}{ EQA-2 } & \multicolumn{2}{|c|}{ EQC-4 } & \multicolumn{2}{|c|}{$E Q C-3 \beta$} & \multicolumn{2}{|c|}{ EQC-3 $\alpha$} \\
\hline & B3LYP & MP2 & B3LYP & MP2 & B3LYP & MP2 & B3LYP & MP2 & B3LYP & MP2 & B3LYP & MP2 \\
\hline $\mathrm{A}^{\mathrm{a}}(\mathrm{MHz})$ & 1611.8 & 1606.5 & 1597.9 & 1595.9 & 1644.7 & 1591.7 & 1581.6 & 1580.1 & 1643.6 & 1652.7 & 1631.6 & 1627.9 \\
\hline $\mathrm{B}(\mathrm{MHz})$ & 637.5 & 639.6 & 640.3 & 643.8 & 596.9 & 614.8 & 646.2 & 649.6 & 599.3 & 600.9 & 602.8 & 606.2 \\
\hline $\mathrm{C}(\mathrm{MHz})$ & 561.2 & 562.5 & 556.3 & 558.7 & 499.6 & 512.8 & 554.1 & 558.2 & 551.7 & 549.1 & 555.1 & 555.4 \\
\hline$\mu_{a}(D)$ & -1.7 & -1.6 & 1.2 & 1.2 & -1.0 & -1.2 & -1.6 & -1.6 & -0.1 & 0.0 & 0.9 & 1.1 \\
\hline$\mu_{b}(D)$ & 0.7 & 0.7 & -1.3 & -1.0 & 1.0 & 0.8 & 0.2 & 0.1 & 1.6 & 1.5 & 1.8 & 1.7 \\
\hline$\mu_{c}(D)$ & 0.7 & 0.7 & 1.2 & 1.2 & 0.2 & 0.2 & 1.2 & 1.2 & 1.2 & 1.2 & 0.9 & 0.8 \\
\hline$\Delta \mathrm{E}\left(\mathrm{cm}^{-1}\right)$ & 0.0 & 20.7 & 50.8 & 78.0 & 139.7 & 284.4 & 90.4 & 197.9 & 223.7 & 320.2 & 221.0 & 302.6 \\
\hline$\Delta \mathrm{E}_{\mathrm{ZPC}}\left(\mathrm{cm}^{-1}\right)$ & 0.0 & 0.0 & 66.1 & 46.3 & 95.9 & 251.7 & 109.1 & 156.7 & 145.3 & 204.3 & 170.8 & 231.3 \\
\hline$\Delta \mathrm{E}(\mathrm{BSSE})\left(\mathrm{kJ} \mathrm{mol} \mathrm{l}^{-1}\right)$ & -20.8 & -14.5 & -20.2 & -13.8 & -19.9 & -13.3 & -20.9 & -14.3 & -19.7 & -13.5 & -19.7 & -13.5 \\
\hline$\Delta \mathrm{E}(\mathrm{SAPT})\left(\mathrm{kJ} \mathrm{mol}^{-1}\right)$ & -16.9 & -17.0 & -16.1 & -16.2 & -15.7 & -16.4 & -16.6 & -16.7 & -15.6 & -15.7 & -15.6 & -15.6 \\
\hline Side view & & & & & & & & & & & & \\
\hline Top view & & & & & & & & & & & & \\
\hline
\end{tabular}

$\overline{\mathrm{a}} \mathrm{A}, \mathrm{B}$ and $\mathrm{C}$ are the rotational constants; $\mu_{\mathrm{a}}, \mu_{\mathrm{b}}$ and $\mu_{\mathrm{c}}$ are the electric dipole moments along the principal inertial axes; $\Delta \mathrm{E}$ are the relative energies; $\Delta \mathrm{E}_{\mathrm{ZPC}}$ are the relative energies including zero-point corrections; $\triangle \mathrm{E}$ (BSSE) are the interaction energies including BSSE and fragment relaxation; $\triangle \mathrm{E}$ (SAPT) are the total interaction energies obtained from SAPT0/juncc-pVDZ calculations on the B3LYP-D3BJ or MP2 structures.

\begin{tabular}{|c|c|c|c|c|c|c|c|c|c|c|c|c|}
\hline & \multicolumn{2}{|c|}{ AXa-3 } & \multicolumn{2}{|c|}{ EQA-1 } & \multicolumn{2}{|c|}{ EQa-4 } & \multicolumn{2}{|c|}{ AXa-4 } & \multicolumn{2}{|c|}{ EQC-2 } & \multicolumn{2}{|c|}{ EQa-3 } \\
\hline & B3LYP & MP2 & B3LYP & MP2 & B3LYP & MP2 & B3LYP & MP2 & B3LYP & MP2 & B3LYP & MP2 \\
\hline $\mathrm{A}^{\mathrm{a}}(\mathrm{MHz})$ & 1276.1 & 1250.1 & 1675.9 & 1680.1 & 1595.7 & 1594.5 & 1571.1 & 1556.1 & 1635.7 & 1613.2 & 1628.1 & 1624.2 \\
\hline $\mathrm{B}(\mathrm{MHz})$ & 856.1 & 871.1 & 581.8 & 583.0 & 614.9 & 623.1 & 689.9 & 703.9 & 599.3 & 612.9 & 608.4 & 612.4 \\
\hline $\mathrm{C}(\mathrm{MHz})$ & 708.4 & 711.5 & 490.9 & 494.6 & 576.9 & 575.8 & 623.7 & 641.6 & 502.3 & 524.1 & 550.9 & 551.8 \\
\hline$\mu_{a}(D)$ & 1.8 & 2.1 & 0.3 & 0.5 & -1.3 & -1.2 & -0.2 & -0.2 & -1.0 & -1.2 & -1.2 & -1.2 \\
\hline$\mu_{\mathrm{b}}(\mathrm{D})$ & 2.3 & 1.6 & 1.4 & 1.3 & 0.7 & 0.6 & -1.3 & -1.4 & -1.0 & -0.2 & -1.3 & -1.2 \\
\hline$\mu_{c}(D)$ & -0.2 & -0.2 & -0.8 & -0.9 & 0.6 & 0.8 & 0.2 & 0.3 & -0.4 & -0.8 & 1.1 & 1.1 \\
\hline$\Delta \mathrm{E}\left(\mathrm{cm}^{-1}\right)$ & 194.1 & 212.6 & 226.6 & 421.0 & 206.8 & 308.7 & 142.1 & 0.0 & 251.1 & 398.3 & 296.0 & 382.1 \\
\hline$\Delta \mathrm{E}_{\mathrm{ZPC}}\left(\mathrm{cm}^{-1}\right)$ & 205.6 & 250.6 & 209.4 & 344.1 & 210.9 & 156.7 & 219.0 & 32.7 & 240.3 & 352.9 & 256.6 & 231.3 \\
\hline$\Delta \mathrm{E}(\mathrm{BSSE})\left(\mathrm{kJ} \mathrm{mol} \mathrm{l}^{-1}\right)$ & -20.2 & -11.7 & -18.8 & -12.1 & -20.9 & -14.1 & -20.9 & -14.3 & -19.6 & -13.4 & -19.8 & -13.6 \\
\hline$\Delta \mathrm{E}(\mathrm{SAPT})\left(\mathrm{kJ} \mathrm{mol}^{-1}\right)$ & -17.0 & -17.0 & -14.9 & -15.7 & -16.6 & -16.8 & -16.6 & -16.8 & -15.8 & -16.0 & -15.5 & -15.7 \\
\hline
\end{tabular}

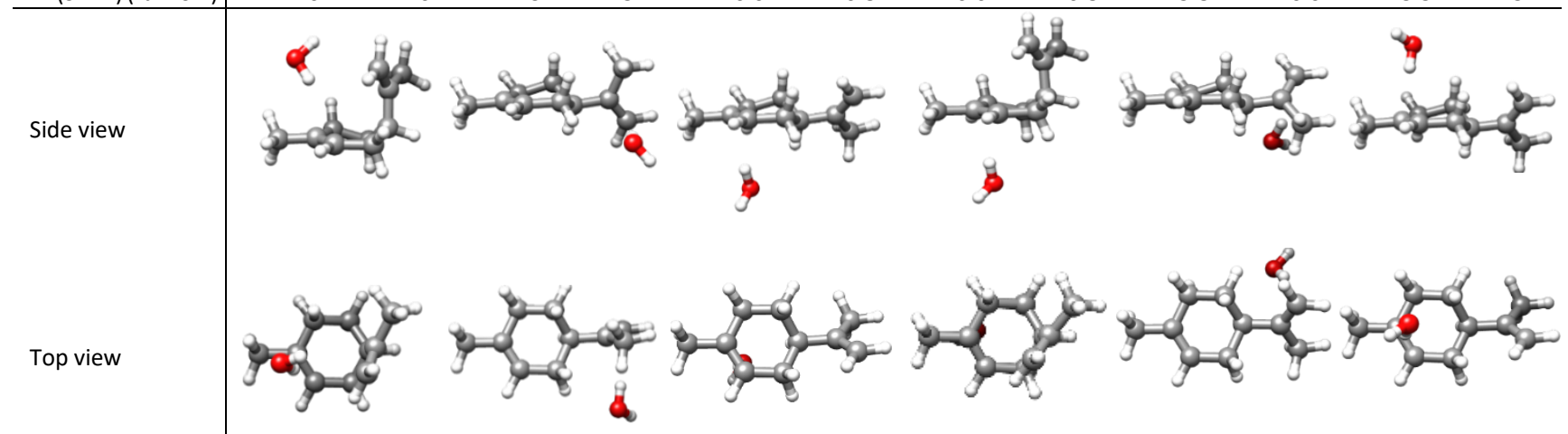

${ }^{\mathrm{a}} \mathrm{A}, \mathrm{B}$ and $\mathrm{C}$ are the rotational constants; $\mu_{\mathrm{a}}, \mu_{\mathrm{b}}$ and $\mu_{\mathrm{c}}$ are the electric dipole moments along the principal inertial axes; $\Delta \mathrm{E}$ are the relative energies; $\Delta \mathrm{E}_{\mathrm{ZPC}}$ are the relative energies including zero-point corrections; $\triangle E$ (BSSE) are the interaction energies including BSSE and fragment relaxation; $\triangle E(S A P T)$ are the total interaction energies obtained from SAPTO/juncc-pVDZ calculations on the B3LYP-D3BJ or MP2 structures. 
Table S1 (cont.). Calculated spectroscopic parameters for the isomers of limonene- $\left(\mathrm{H}_{2} \mathrm{O}\right)$ at $\mathrm{MP2} / 6-311++\mathrm{G}(\mathrm{d}, \mathrm{p})$ and B3LYP-D3BJ/6-311++G(d,p) level of theory.

\begin{tabular}{|c|c|c|c|c|c|c|c|c|c|c|c|c|}
\hline & \multicolumn{2}{|c|}{ AXa-2 $\alpha$} & \multicolumn{2}{|c|}{ EQC-1 } & \multicolumn{2}{|c|}{ AXC-2 } & \multicolumn{2}{|c|}{ EQa-2 } & \multicolumn{2}{|c|}{ AXa-1 } & \multicolumn{2}{|c|}{ EQa-1 } \\
\hline & B3LYP & MP2 & B3LYP & MP2 & B3LYP & MP2 & B3LYP & MP2 & B3LYP & MP2 & B3LYP & MP2 \\
\hline $\mathrm{A}^{\mathrm{a}}(\mathrm{MHz})$ & 1215.6 & 1190.7 & 1654.4 & 1655.8 & 1312.9 & 1245.5 & 1662.3 & 1648.8 & 1602.1 & 1633.1 & 1633.4 & 1637.2 \\
\hline $\mathrm{B}(\mathrm{MHz})$ & 905.6 & 927.4 & 573.6 & 573.6 & 853.3 & 897.8 & 585.1 & 589.3 & 662.9 & 666.4 & 569.9 & 572.3 \\
\hline $\mathrm{C}(\mathrm{MHz})$ & 720.2 & 728.0 & 534.4 & 531.3 & 739.0 & 752.5 & 494.7 & 500.6 & 586.6 & 600.1 & 552.4 & 550.4 \\
\hline$\mu_{\mathrm{a}}(\mathrm{D})$ & 1.7 & 1.6 & -0.1 & -0.2 & -0.2 & -0.5 & -0.8 & -1.0 & 0.2 & 0.1 & 0.5 & 0.9 \\
\hline$\mu_{\mathrm{b}}(\mathrm{D})$ & 0.3 & 0.2 & 1.4 & 1.3 & 3.0 & 2.8 & 1.4 & 1.4 & -1.3 & -1.0 & -1.1 & -0.6 \\
\hline$\mu_{\mathrm{c}}(\mathrm{D})$ & -0.4 & -0.6 & 0.8 & 0.9 & 0.2 & 0.0 & -0.5 & -0.4 & -1.2 & -1.4 & 0.3 & 0.7 \\
\hline$\Delta \mathrm{E}\left(\mathrm{cm}^{-1}\right)$ & 243.2 & 192.3 & 290.5 & 528.6 & 176.3 & 330.7 & 408.0 & 593.3 & 310.1 & 212.0 & 404.7 & 704.7 \\
\hline$\Delta \mathrm{E}_{\mathrm{ZPC}}\left(\mathrm{cm}^{-1}\right)$ & 302.4 & 193.4 & 315.2 & 513.8 & 337.6 & 482.2 & 339.7 & 352.9 & 347.9 & 175.1 & 418.1 & 617.2 \\
\hline$\Delta \mathrm{E}(\mathrm{BSSE})\left(\mathrm{kJ} \mathrm{\textrm {mol } ^ { - 1 } )}\right.$ & -19.7 & -12.3 & -18.9 & -12.0 & -26.6 & -15.7 & -19.1 & -12.8 & -19.4 & -12.8 & -18.9 & -11.2 \\
\hline$\Delta \mathrm{E}(\mathrm{SAPT})\left(\mathrm{kJ} \mathrm{mol}^{-1}\right)$ & -16.9 & -17.4 & -14.6 & -15.1 & -20.9 & -20.6 & -15.1 & -15.3 & -15.6 & -16.0 & -14.9 & -15.2 \\
\hline
\end{tabular}

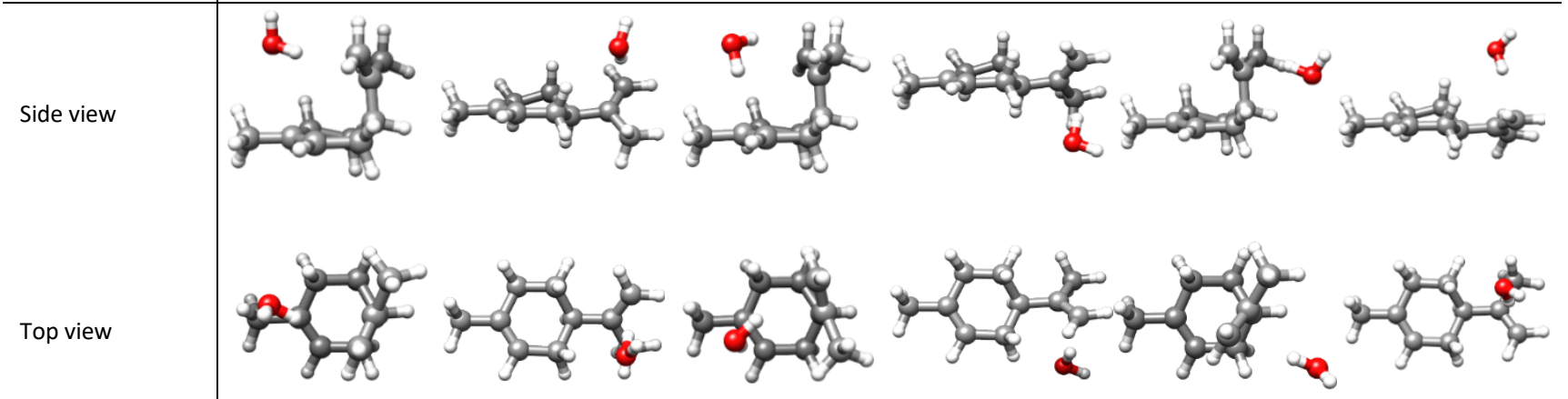

$\overline{\mathrm{a}} \mathrm{A}, \mathrm{B}$ and $\mathrm{C}$ are the rotational constants; $\mu_{\mathrm{a}} \mu_{\mathrm{b}}$ and $\mu_{\mathrm{c}}$ are the electric dipole moments along the principal inertial axes; $\Delta \mathrm{E}$ are the relative energies; $\Delta \mathrm{E}_{\mathrm{ZPC}}$ are the relative energies including zero-point corrections; $\triangle E$ (BSSE) are the interaction energies including BSSE and fragment relaxation; $\triangle E(S A P T)$ are the total interaction energies obtained from SAPT0/juncc-pVDZ calculations on the B3LYP-D3BJ or MP2 structures.

\begin{tabular}{|c|c|c|c|c|c|c|c|c|c|c|c|c|}
\hline & \multicolumn{2}{|c|}{$A \times a-2 \beta$} & \multicolumn{2}{|c|}{ AXA-1 } & \multicolumn{2}{|c|}{ AXA-2 } & \multicolumn{2}{|c|}{ AXC-4 } & \multicolumn{2}{|c|}{ AXC-1 } & \multicolumn{2}{|c|}{ AXA-4 } \\
\hline & B3LYP & MP2 & B3LYP & MP2 & B3LYP & MP2 & B3LYP & MP2 & B3LYP & MP2 & B3LYP & MP2 \\
\hline $\mathrm{A}^{\mathrm{a}}(\mathrm{MHz})$ & 1232.8 & 1197.1 & 1367.5 & 1320.9 & 1638.4 & 1632.6 & 1530.9 & 1505.3 & 1686.9 & 1671.7 & 1575.2 & 1570.1 \\
\hline $\mathrm{B}(\mathrm{MHz})$ & 881.8 & 902.9 & 783.2 & 810.4 & 654.2 & 668.8 & 681.6 & 708.3 & 625.7 & 641.0 & 705.8 & 717.9 \\
\hline $\mathrm{C}(\mathrm{MHz})$ & 715.5 & 717.7 & 650.4 & 662.8 & 596.6 & 614.3 & 644.5 & 672.6 & 583.8 & 605.8 & 615.6 & 626.9 \\
\hline$\mu_{a}(D)$ & -0.4 & -0.2 & 0.8 & 0.9 & -0.1 & -0.4 & 0.1 & 0.2 & -0.2 & -0.1 & 0.7 & 0.6 \\
\hline$\mu_{\mathrm{b}}(\mathrm{D})$ & 2.5 & 2.2 & -0.8 & -0.8 & -1.3 & -1.3 & -0.8 & -1.1 & -0.8 & -0.5 & -1.8 & -1.7 \\
\hline$\mu_{c}(D)$ & 0.3 & 0.6 & 0.4 & 0.7 & -1.0 & -0.8 & 1.6 & 1.4 & 1.4 & 1.5 & 0.8 & 0.8 \\
\hline$\Delta \mathrm{E}\left(\mathrm{cm}^{-1}\right)$ & 212.0 & 256.7 & 692.0 & 753.8 & 736.1 & 734.2 & 794.4 & 654.8 & 955.6 & 911.5 & 666.7 & 591.3 \\
\hline$\Delta \mathrm{E}_{\mathrm{ZPC}}\left(\mathrm{cm}^{-1}\right)$ & 461.1 & 262.1 & 724.7 & 780.9 & 727.1 & 750.6 & 864.3 & 752.6 & 987.0 & 987.0 & 989.0 & 651.2 \\
\hline$\Delta \mathrm{E}(\mathrm{BSSE})\left(\mathrm{kJ} \mathrm{mol} \mathrm{m}^{-1}\right)$ & -19.3 & -11.2 & -20.7 & -13.0 & -20.1 & -13.7 & -20.7 & -14.5 & -19.3 & -13.1 & -20.3 & -13.9 \\
\hline$\Delta \mathrm{E}(\mathrm{SAPT})\left(\mathrm{kJ} \mathrm{mol}^{-1}\right)$ & -16.2 & -16.6 & -16.5 & -16.3 & -16.0 & -16.5 & -16.5 & -16.7 & -15.7 & -16.0 & -16.0 & -16.1 \\
\hline
\end{tabular}

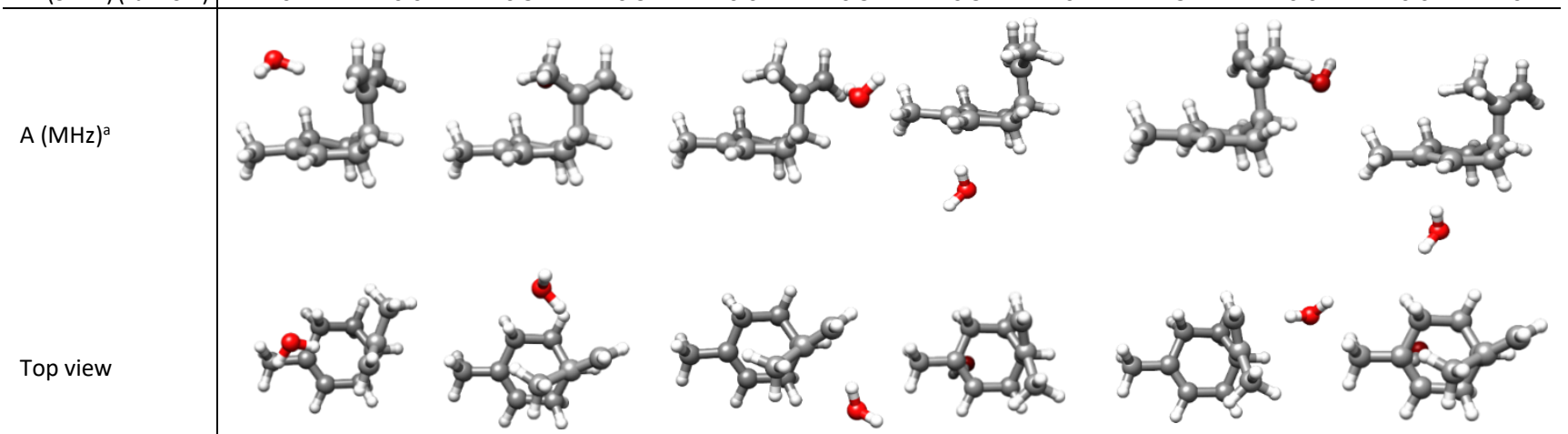

${ }^{a} \mathrm{~A}, \mathrm{~B}$ and $\mathrm{C}$ are the rotational constants; $\mu_{\mathrm{a}}, \mu_{\mathrm{b}}$ and $\mu_{\mathrm{c}}$ are the electric dipole moments along the principal inertial axes; $\Delta \mathrm{E}$ are the relative energies; $\Delta \mathrm{E}_{\mathrm{ZPC}}$ are the relative energies including zero-point corrections; $\triangle E$ (BSSE) are the interaction energies including BSSE and fragment relaxation; $\triangle E(S A P T)$ are the total interaction energies obtained from SAPTO/juncc-pVDZ calculations on the B3LYP-D3BJ or MP2 structures. 
Table S2. Experimental spectroscopic parameters of the observed isotopic species of isomer 1, EQA-3.

\begin{tabular}{l|cccc}
\hline \multicolumn{1}{c|}{ Parameter } & DOH & HOD & DOD & $\mathbf{H}_{\mathbf{2}} \mathbf{1 8}^{\mathbf{2}}$ \\
\hline \hline $\mathrm{A}^{\mathrm{a}}(\mathrm{MHz})$ & $1567.0817(46)^{\mathrm{b}}$ & $1534.1825(52)$ & $1523.0854(61)$ & $1517.17489(56)$ \\
$\mathrm{B}(\mathrm{MHz})$ & $630.86535(93)$ & $629.58137(89)$ & $626.6900(11)$ & $628.55703(34)$ \\
$\mathrm{C}(\mathrm{MHz})$ & $550.25403(69)$ & $545.20129(68)$ & $541.77564(84)$ & $542.05245(29)$ \\
$\Delta_{\mathrm{J}}(\mathrm{kHz})$ & $0.1225(58)$ & $0.1109(55)$ & $0.1117(60)$ & $0.1294(38)$ \\
$\Delta_{\mathrm{J}}(\mathrm{kHz})$ & $1.252(34)$ & $1.339(34)$ & $1.129(37)$ & $1.343(27)$ \\
$\delta_{\mathrm{J}}(\mathrm{kHz})$ & $0.0315(63)$ & $0.0298(58)$ & $0.0291(69)$ & $0.0344(31)$ \\
$\sigma^{c}(\mathrm{kHz})$ & 5.2 & 4.6 & 6.0 & 4.3 \\
$\mathrm{~N}^{\mathrm{d}}$ & 31 & 28 & 34 & 51 \\
\hline
\end{tabular}

${ }^{a} \mathrm{~A}, \mathrm{~B}$ and $\mathrm{C}$ are the rotational constants; $\Delta_{\mathrm{J}}, \Delta_{\mathrm{J}}$ and $\delta_{\mathrm{J}}$ are the quartic centrifugal distortion constants. ${ }^{\mathrm{b}}$ Standard error in parentheses in the units of the last digit. ${ }^{\mathrm{C}}$ Rms deviation of the fit. ${ }^{\mathrm{d}}$ Number of rotational transitions included in the fit.

Table S3. Experimental spectroscopic parameters of the observed isotopic species of isomer 2, EQA-4.

\begin{tabular}{l|cccc}
\hline \multicolumn{1}{c|}{ Parameter } & DOH & HOD & DOD & $\mathbf{H}_{\mathbf{2}} \mathbf{1 8}^{\mathbf{1 8}}$ \\
\hline \hline $\mathrm{A}^{\mathrm{a}}(\mathrm{MHz})$ & $1557.8491(16)$ & $1524.7558(15)$ & $1513.5118(12)$ & $1508.64203(77)$ \\
$\mathrm{B}(\mathrm{MHz})$ & $631.98633(76)$ & $630.71574(47)$ & $627.98805(43)$ & $629.05728(35)$ \\
$\mathrm{C}(\mathrm{MHz})$ & $545.32986(65)$ & $540.46031(40)$ & $537.06807(39)$ & $537.12952(32)$ \\
$\Delta_{\mathrm{J}}(\mathrm{kHz})$ & $0.1011(79)$ & $0.0815(57)$ & $0.0994(49)$ & $0.0987(30)$ \\
$\Delta_{\mathrm{J}}(\mathrm{kHz})$ & $1.091(87)$ & $1.007(62)$ & $0.926(60)$ & $1.035(43)$ \\
$\delta_{\mathrm{J}}(\mathrm{kHz})$ & $0.0376(68)$ & $0.0188(40)$ & $0.0338(37)$ & $0.0283(26)$ \\
$\sigma^{c}(\mathrm{kHz})$ & 7.7 & 5.6 & 4.7 & 4.8 \\
$\mathrm{~N}^{\mathrm{d}}$ & 36 & 42 & 36 & 48 \\
\hline
\end{tabular}

${ }^{\mathrm{a}} \mathrm{A}, \mathrm{B}$ and $\mathrm{C}$ are the rotational constants; $\Delta_{\mathrm{\jmath}}, \Delta_{\mathrm{\jmath}}$ and $\delta_{\mathrm{\jmath}}$ are the quartic centrifugal distortion constants. ${ }^{\mathrm{b}}$ Standard error in parentheses in the units of the last digit. ${ }^{\mathrm{C}} \mathrm{Rms}$ deviation of the fit. ${ }^{\mathrm{d}}$ Number of rotational transitions included in the fit.

Table S4. Experimental spectroscopic parameters of the observed isotopic species of isomer 3, EQC-4.

\begin{tabular}{l|cccc}
\hline \multicolumn{1}{c|}{ Parameter } & DOH & HOD & DOD & $\mathbf{H}_{\mathbf{2}}{ }^{18} \mathbf{O}$ \\
\hline \hline $\mathrm{A}^{\mathrm{a}}(\mathrm{MHz})$ & $1536.0002(14)^{\mathrm{b}}$ & $1502.5268(16)$ & $1490.97469(94)$ & $1484.80563(69)$ \\
$\mathrm{B}(\mathrm{MHz})$ & $642.05714(70)$ & $640.5953(10)$ & $637.98107(52)$ & $639.36216(40)$ \\
$\mathrm{C}(\mathrm{MHz})$ & $546.52885(68)$ & $541.98754(88)$ & $538.71089(50)$ & $539.64218(37)$ \\
$\Delta_{\mathrm{J}}(\mathrm{kHz})$ & $0.0948(76)$ & $0.1012(99)$ & $0.1050(59)$ & $0.1077(43)$ \\
$\Delta_{\mathrm{J}}(\mathrm{kHz})$ & $1.088(49)$ & $1.143(65)$ & $1.038(39)$ & $1.131(31)$ \\
$\delta_{\mathrm{J}}(\mathrm{kHz})$ & $0.0286(59)$ & $0.0578(84)$ & $0.0445(42)$ & $0.0343(32)$ \\
$\sigma^{c}(\mathrm{kHz})$ & 7.3 & 9.1 & 5.4 & 4.3 \\
$\mathrm{~N}^{\mathrm{d}}$ & 34 & 31 & 34 & 39 \\
\hline
\end{tabular}

${ }^{\mathrm{a}} \mathrm{A}, \mathrm{B}$ and $\mathrm{C}$ are the rotational constants; $\Delta_{\mathrm{\jmath}}, \Delta_{\mathrm{\jmath}}$ and $\delta_{\mathrm{\jmath}}$ are the quartic centrifugal distortion constants. ${ }^{\mathrm{b}}$ Standard error in parentheses in the units of the last digit. ${ }^{\mathrm{C}} \mathrm{Rms}$ deviation of the fit. ${ }^{\mathrm{d}}$ Number of rotational transitions included in the fit.

Table S5. Experimental spectroscopic parameters of the observed isotopic species of isomer 4, EQC-2.

\begin{tabular}{l|cccc}
\multicolumn{1}{c|}{ Parameter } & DOH & HOD & DOD & $\mathbf{H}^{\mathbf{2}^{\mathbf{1 8}} \mathbf{O}}$ \\
\hline \hline $\mathrm{A}^{\mathrm{a}}(\mathrm{MHz})$ & $1600.1043(21)^{\mathrm{b}}$ & $1569.230(54)$ & $1561.3588(43)$ & $1554.2493(18)$ \\
$\mathrm{B}(\mathrm{MHz})$ & $593.94202(42)$ & $591.81289(78)$ & $586.61105(48)$ & $588.74168(53)$ \\
$\mathrm{C}(\mathrm{MHz})$ & $501.75930(54)$ & $498.32829(79)$ & $493.45062(47)$ & $495.14239(60)$ \\
$\Delta_{\mathrm{J}}(\mathrm{kHz})$ & $0.1109(64)$ & $0.1108(86)$ & $0.1213(53)$ & $0.1103(68)$ \\
$\Delta_{\mathrm{J}}(\mathrm{kHz})$ & $0.539(52)$ & {$[0.633]^{\mathrm{c}}$} & $0.337(91)$ & {$[0.633]^{\mathrm{c}}$} \\
$\sigma^{\mathrm{d}}(\mathrm{kHz})$ & 6.5 & 9.3 & 6.6 & 7.6 \\
$\mathrm{~N}^{\mathrm{e}}$ & 26 & 20 & 28 & 24 \\
\hline
\end{tabular}

${ }^{\mathrm{a}} \mathrm{A}, \mathrm{B}$ and $\mathrm{C}$ are the rotational constants; $\Delta_{\mathrm{J}}$ and $\Delta_{\mathrm{J}}$ are the quartic centrifugal distortion constants. ${ }^{\mathrm{b}}$ Standard error in parentheses in the units of the last digit. ${ }^{c}$ Fixed to the value of the parent species. ${ }^{\mathrm{d}} \mathrm{Rms}$ deviation of the fit. eNumber of rotational transitions included in the fit. 
Table S6. Experimental spectroscopic parameters of the observed isotopic species of isomer 6, EQC-3ß.

\begin{tabular}{l|cccc}
\hline \multicolumn{1}{c|}{ Parameter } & DOH & HOD & DOD & H $^{\mathbf{1 8} O}$ \\
\hline \hline $\mathrm{A}^{\mathrm{a}}(\mathrm{MHz})$ & $1620.3857(40)^{\mathrm{b}}$ & $1588.0560(18)$ & $1576.2492(34)$ & $1575.3723(19)$ \\
$\mathrm{B}(\mathrm{MHz})$ & $588.92593(90)$ & $586.77219(67)$ & $583.57313(53)$ & $584.1291(10)$ \\
$\mathrm{C}(\mathrm{MHz})$ & $540.7356(12)$ & $536.04225(78)$ & $533.03403(57)$ & $531.64208(89)$ \\
$\Delta_{\mathrm{J}}(\mathrm{kHz})$ & $0.119(15)$ & $0.136(13)$ & $0.1265(83)$ & $0.153(18)$ \\
$\Delta_{\mathrm{J}}(\mathrm{kHz})$ & $0.82(30)$ & $0.99(10)$ & $0.69(16)$ & $0.81(11)$ \\
$\sigma^{c}(\mathrm{kHz})$ & 9.3 & 6.0 & 6.8 & 7.2 \\
$\mathrm{~N}^{\mathrm{d}}$ & 14 & 18 & 17 & 22 \\
\hline
\end{tabular}

${ }^{a} \mathrm{~A}, \mathrm{~B}$ and $\mathrm{C}$ are the rotational constants; $\Delta_{\mathrm{\jmath}}$ and $\Delta_{\mathrm{\jmath}}$ are the quartic centrifugal distortion constants. ${ }^{\mathrm{b}}$ Standard error in parentheses in the units of the last digit. ${ }^{\mathrm{C}} \mathrm{Rms}$ deviation of the fit. ${ }^{\mathrm{d}}$ Number of rotational transitions included in the fit.

Table S7. Experimental spectroscopic parameters of the observed isotopic species of isomer 7, AXa-2 $\alpha$.

\begin{tabular}{l|ccc}
\hline \multicolumn{1}{c|}{ Parameter } & DOH & DOD & $\mathbf{H}^{\mathbf{1}^{\mathbf{1 8}} \mathbf{O}}$ \\
\hline \hline $\mathrm{A}^{\mathrm{a}}(\mathrm{MHz})$ & $1162.8939(70)^{\mathrm{b}}$ & $1129.8997(73)$ & $1133.855(20)$ \\
$\mathrm{B}(\mathrm{MHz})$ & $904.42160(11)$ & $903.53553(17)$ & $899.5281(12)$ \\
$\mathrm{C}(\mathrm{MHz})$ & $700.31986(24)$ & $687.98729(95)$ & $686.36570(95)$ \\
$\Delta_{\mathrm{J}}(\mathrm{kHz})$ & $0.1589(24)$ & $0.16391(10)$ & {$[0]$.} \\
$\Delta_{\mathrm{J}}(\mathrm{kHz})$ & $0.730(16)$ & $0.75268(10)$ & {$[0]$.} \\
$\sigma^{c}(\mathrm{kHz})$ & 6.3 & 10.1 & 6.4 \\
$\mathrm{~N}^{\mathrm{d}}$ & 16 & 9 & 8 \\
\hline
\end{tabular}

${ }^{a} \mathrm{~A}, \mathrm{~B}$ and $\mathrm{C}$ are the rotational constants; $\Delta_{\mathrm{\jmath}}$ and $\Delta_{\mathrm{\jmath}}$ are the quartic centrifugal distortion constants. ${ }^{\mathrm{b}}$ Standard error in parentheses in the units of the last digit. ${ }^{\mathrm{C}}$ Rms deviation of the fit. ${ }^{\mathrm{d}}$ Number of rotational transitions included in the fit. 
Table S8. Experimental substitution coordinates of the hydrogen and oxygen atoms of water in limonene- $\mathrm{H}_{2} \mathrm{O}$ complexes and the corresponding equilibrium coordinates from MP2 and B3LYP-D3BJ calculations using the 6$311++G(d, p)$ basis set.

\begin{tabular}{|c|c|c|c|c|c|c|c|c|c|}
\hline & \multicolumn{3}{|c|}{ Isomer 1 - EQA-3 } & \multicolumn{3}{|c|}{ Isomer 2 - EQA-4 } & \multicolumn{3}{|c|}{ Isomer 3 - EQC-4 } \\
\hline & $r_{\mathrm{s}}$ & B3LYP & MP2 & $r_{\mathrm{s}}$ & B3LYP & MP2 & $r_{\mathrm{s}}$ & B3LYP & MP2 \\
\hline & \multicolumn{3}{|c|}{01} & \multicolumn{3}{|c|}{01} & \multicolumn{3}{|c|}{01} \\
\hline$|a|^{a}$ & $1.79569(84)^{b}$ & 1.679 & 1.700 & $1.85654(81)$ & 1.697 & 1.688 & $1.55370(97)$ & 1.486 & 1.447 \\
\hline$|b|$ & $2.60411(58)$ & 2.576 & 2.574 & $2.59115(58)$ & 2.579 & 2.577 & $2.52020(60)$ & 2.464 & 2.470 \\
\hline \multirow[t]{2}{*}{$|c|$} & $0.0000(91)$ & 0.017 & 0.048 & $0.2323(65)$ & 0.296 & 0.298 & $0.9240(16)$ & 0.987 & 0.976 \\
\hline & \multicolumn{3}{|c|}{$\mathrm{H} 1$} & \multicolumn{3}{|c|}{$\mathrm{H} 1$} & \multicolumn{3}{|c|}{$\mathrm{H} 1$} \\
\hline$|a|$ & $1.85159(85)$ & 1.784 & 1.808 & $1.83059(85)$ & 1.751 & 1.763 & $1.73760(90)$ & 1.636 & 1.621 \\
\hline$|b|$ & $1.5697(10)$ & 1.671 & 1.671 & $1.61743(96)$ & 1.722 & 1.737 & $1.63647(95)$ & 1.748 & 1.769 \\
\hline \multirow{2}{*}{$|c|$} & $0.3589(44)$ & 0.313 & 0.374 & $0.134(12)$ & 0.153 & 0.174 & $0.3231(48)$ & 0.351 & 0.334 \\
\hline & \multicolumn{3}{|c|}{$\mathrm{H} 2$} & \multicolumn{3}{|c|}{$\mathrm{H} 2$} & \multicolumn{3}{|c|}{$\mathrm{H} 2$} \\
\hline$|a|$ & $2.22753(71)$ & 2.432 & 2.438 & $2.17533(70)$ & 2.288 & 2.269 & $2.01376(79)$ & 2.167 & 2.112 \\
\hline$|b|$ & $3.07160(52)$ & 3.062 & 3.046 & $3.09407(49)$ & 3.158 & 3.163 & $3.05520(52)$ & 3.116 & 3.132 \\
\hline$|c|$ & $0.3965(40)$ & 0.332 & 0.441 & $0.4275(36)$ & 0.195 & 0.193 & $0.9228(17)$ & 0.796 & 0.769 \\
\hline
\end{tabular}

a Absolute values of the $a, b, c$ coordinates in $\AA$.

${ }^{b}$ Errors in parentheses, including Costain's error, in units of the last digit.

\begin{tabular}{|c|c|c|c|c|c|c|}
\hline & \multicolumn{3}{|c|}{ Isomer 4-EQC-2 } & \multicolumn{3}{|c|}{ EQA-2 } \\
\hline & $\bar{r}_{\mathrm{s}}$ & B3LYP & MP2 & $\bar{r}_{\mathrm{s}}$ & B3LYP & MP2 \\
\hline & \multicolumn{3}{|c|}{ O1 } & \multicolumn{3}{|c|}{ O1 } \\
\hline$|a|^{a}$ & $2.60514(58)^{b}$ & 2.667 & 2.341 & $2.60514(58)$ & 2.812 & 2.542 \\
\hline$|b|$ & $2.25469(67)$ & 2.226 & 2.261 & $2.25469(67)$ & 2.304 & 2.421 \\
\hline$|c|$ & $0.8401(18)$ & 0.823 & 0.951 & $0.8401(18)$ & 0.270 & 0.328 \\
\hline & \multicolumn{3}{|c|}{$\mathrm{H} 1$} & \multicolumn{3}{|c|}{$\mathrm{H} 1$} \\
\hline$|a|$ & $2.80236(56)$ & 2.711 & 2.543 & $2.80236(56)$ & 2.832 & 2.682 \\
\hline$|b|$ & $1.5152(10)$ & 1.456 & 1.582 & $1.5152(10)$ & 1.357 & 1.481 \\
\hline$|c|$ & $0.0000(24)$ & 0.237 & 0.296 & $0.0000(24)$ & 0.475 & 0.499 \\
\hline & \multicolumn{3}{|c|}{$\mathrm{H} 2$} & \multicolumn{3}{|c|}{$\mathrm{H} 2^{\mathrm{b}}$} \\
\hline$|a|$ & $3.1029(10)$ & 3.578 & 3.102 & $3.1029(10)$ & 3.728 & 3.378 \\
\hline$|b|$ & $2.7201(12)$ & 2.516 & 2.846 & $2.7201(12)$ & 2.594 & 2.822 \\
\hline$|c|$ & $0.9042(37)$ & 0.924 & 0.909 & $0.9042(37)$ & 0.320 & 0.579 \\
\hline
\end{tabular}

a Absolute values of the $a, b, c$ coordinates in $\AA$.

b Errors in parentheses, including Costain's error, in units of the last digit.

\begin{tabular}{|c|c|c|c|c|c|c|}
\hline & \multicolumn{3}{|c|}{ Isomer 6 - EQC-3 $\beta$} & \multicolumn{3}{|c|}{ EQC-3 $\alpha$} \\
\hline & $r_{\mathrm{s}}$ & B3LYP & MP2 & $r_{\mathrm{s}}$ & B3LYP & MP2 \\
\hline & \multicolumn{3}{|c|}{01} & \multicolumn{3}{|c|}{ O1 } \\
\hline$|a|^{a}$ & $2.31664(66)^{b}$ & 2.173 & 2.246 & $2.31664(66)$ & 2.047 & 2.059 \\
\hline$|b|$ & $2.30357(67)$ & 2.319 & 2.260 & $2.30357(67)$ & 2.381 & 2.362 \\
\hline$|c|$ & $0.7631(20)$ & 0.778 & 0.836 & $0.7631(20)$ & 0.744 & 0.791 \\
\hline & \multicolumn{3}{|c|}{$\mathrm{H} 1$} & \multicolumn{3}{|c|}{$\mathrm{H} 1$} \\
\hline$|a|$ & $1.94086(86)$ & 2.003 & 2.041 & $1.94086(86)$ & 1.947 & 1.979 \\
\hline$|b|$ & $1.2230(14)$ & 1.373 & 1.322 & $1.2230(14)$ & 1.421 & 1.403 \\
\hline$|c|$ & $1.0179(16)$ & 0.894 & 0.941 & $1.0179(16)$ & 0.830 & 0.873 \\
\hline & \multicolumn{3}{|c|}{$\mathrm{H} 2$} & \multicolumn{3}{|c|}{$\mathrm{H} 2$} \\
\hline$|a|$ & $2.48519(64)$ & 2.224 & 2.401 & $2.48519(64)$ & 2.654 & 2.788 \\
\hline$|b|$ & $2.66858(60)$ & 2.678 & 2.558 & $2.66858(60)$ & 2.634 & 2.578 \\
\hline$|c|$ & $1.3581(12)$ & 1.668 & 1.735 & $1.3581(12)$ & 1.445 & 1.378 \\
\hline
\end{tabular}

a Absolute values of the $a, b, c$ coordinates in $\AA$.

${ }^{\mathrm{b}}$ Errors in parentheses, including Costain's error, in units of the last digit. 
Table S8 (cont.). Experimental substitution coordinates of the hydrogen and oxygen atoms of water in limonene$\mathrm{H}_{2} \mathrm{O}$ complexes compared with equilibrium coordinates from MP2 and B3LYP-D3BJ calculations using the 6$311++G(d, p)$ basis set.

\begin{tabular}{|c|c|c|c|c|c|c|}
\hline & \multicolumn{3}{|c|}{ Isomer $7-\mathrm{AXa}-2 \alpha$} & \multicolumn{3}{|c|}{ AXa-3 } \\
\hline & $r_{\mathrm{s}}$ & B3LYP & MP2 & $r_{\mathrm{s}}$ & B3LYP & MP2 \\
\hline & \multicolumn{3}{|c|}{01} & \multicolumn{3}{|c|}{01} \\
\hline$|a|^{a}$ & $0.8580(21)^{b}$ & 0.604 & 0.700 & $0.8580(21)$ & 1.151 & 1.459 \\
\hline$|b|$ & $2.92208(63)$ & 2.855 & 2.872 & $2.92208(63)$ & 2.718 & 2.667 \\
\hline \multirow[t]{2}{*}{$|c|$} & $0.116(16)$ & 0.011 & 0.026 & $0.116(16)$ & 0.172 & 0.111 \\
\hline & \multicolumn{3}{|c|}{$\mathrm{H} 1$} & \multicolumn{3}{|c|}{$\mathrm{H} 1$} \\
\hline$|a|$ & $0.0000(14)$ & 0.085 & 0.191 & $0.0000(14)$ & 1.192 & 1.244 \\
\hline$|b|$ & $2.28685(77)$ & 2.119 & 2.147 & $2.28685(77)$ & 1.854 & 1.803 \\
\hline \multirow[t]{2}{*}{$|c|$} & $0.5312(33)$ & 0.347 & 0.410 & $0.5312(33)$ & 0.260 & 0.260 \\
\hline & \multicolumn{3}{|c|}{$\mathrm{H} 2^{\mathrm{c}}$} & \multicolumn{3}{|c|}{$\mathrm{H} 2^{\mathrm{c}}$} \\
\hline$|a|$ & $0.6601(28)$ & 0.267 & 0.347 & $0.6601(28)$ & 0.207 & 0.590 \\
\hline$|b|$ & $3.51389(51)$ & 3.635 & 3.648 & $3.51389(51)$ & 2.841 & 3.058 \\
\hline$|c|$ & $0.3721(47)$ & 0.440 & 0.469 & $0.3721(47)$ & 0.318 & 0.229 \\
\hline
\end{tabular}

a Absolute values of the $a, b, c$ coordinates in $\AA$.

${ }^{\mathrm{b}}$ Errors in parentheses, including Costain's error, in units of the last digit.

${ }^{c}$ Experimental $r_{s}$ coordinates determined considering the $\mathrm{D}_{2} \mathrm{O}$ species as the parent species. 
Table S9. Intermolecular stabilising energy contributions $\left(\geq 0.42 \mathrm{~kJ} \mathrm{~mol}^{-1}\right)$ for isomer EQA-3 of limonene- $\mathrm{H}_{2} \mathrm{O}$ from Natural Bond Orbital (NBO) analysis at the B3LYP-D3BJ/6-311++G(d,p) level of theory.

\begin{tabular}{|c|c|c|}
\hline \multicolumn{3}{|c|}{ From limonene to water } \\
\hline Donor & Acceptor & $\mathrm{kJ} / \mathrm{mol}$ \\
\hline $\mathrm{BD}(1) \mathrm{C} 2-\mathrm{C} 3$ & $\mathrm{RY} *(3) \mathrm{H} 29$ & 0.79 \\
\hline $\mathrm{BD}(2) \mathrm{C} 2-\mathrm{C} 3$ & $\mathrm{RY} *(4) \mathrm{H} 29$ & 0.71 \\
\hline $\mathrm{BD}(2) \mathrm{C} 2-\mathrm{C} 3$ & $\mathrm{BD} *(1) \mathrm{O} 27-\mathrm{H} 29$ & 13.77 \\
\hline $\mathrm{BD}(1) \mathrm{C} 5-\mathrm{H} 17$ & $R Y^{*}(1) \mathrm{O} 27$ & 0.88 \\
\hline $\mathrm{BD}(1) \mathrm{C} 5-\mathrm{H} 17$ & $\mathrm{BD}^{*}(1) \mathrm{O} 27-\mathrm{H} 28$ & 0.42 \\
\hline \multicolumn{3}{|c|}{ From water to limonene } \\
\hline Donor & Acceptor & $\mathrm{kJ} / \mathrm{mol}$ \\
\hline $\mathrm{BD}(1) \mathrm{O} 27-\mathrm{H} 28$ & $\mathrm{RY}^{*}(1) \mathrm{H} 17$ & 1.92 \\
\hline LP (1) O27 & $\mathrm{BD} *(1) \mathrm{C} 5-\mathrm{H} 17$ & 1.21 \\
\hline $\mathrm{LP}(2) \mathrm{O} 27$ & $\mathrm{BD}^{*}(2) \mathrm{C} 2-\mathrm{C} 3$ & 1.21 \\
\hline
\end{tabular}

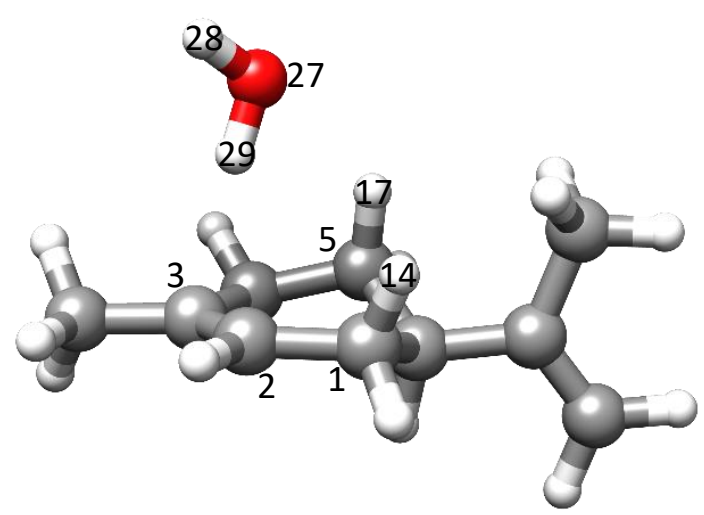

Table S10. Intermolecular stabilising energy contributions $\left(\geq 0.42 \mathrm{~kJ} \mathrm{~mol}^{-1}\right)$ for isomer EQA-4 of limonene- $\mathrm{H}_{2} \mathrm{O}$ from Natural Bond Orbital (NBO) analysis at the B3LYP-D3BJ/6-311++G(d,p) level of theory.

\begin{tabular}{|c|c|c|}
\hline \multicolumn{3}{|c|}{ From limonene to water } \\
\hline Donor & Acceptor & $\mathrm{kJ} / \mathrm{mol}$ \\
\hline $\mathrm{BD}(1) \mathrm{C} 1-\mathrm{H} 16$ & $\mathrm{BD}^{*}(1) \mathrm{O} 11-\mathrm{H} 28$ & 0.42 \\
\hline $\mathrm{BD}(1) \mathrm{C} 3-\mathrm{C} 4$ & $\mathrm{RY}^{*}(3) \mathrm{H} 29$ & 0.84 \\
\hline $\mathrm{BD}(2) \mathrm{C} 3-\mathrm{C} 4$ & $\mathrm{RY}^{*}(4) \mathrm{H} 29$ & 0.71 \\
\hline $\mathrm{BD}(2) \mathrm{C} 3-\mathrm{C} 4$ & $\mathrm{BD}^{*}(1) \mathrm{O} 11-\mathrm{H} 29$ & 13.05 \\
\hline \multicolumn{3}{|c|}{ From water to limonene } \\
\hline Donor & Acceptor & $\mathrm{kJ} / \mathrm{mol}$ \\
\hline $\mathrm{BD}(1) \mathrm{O} 11-\mathrm{H} 28$ & $\mathrm{RY} *(1) \mathrm{H} 16$ & 1.30 \\
\hline $\mathrm{BD}(1) \mathrm{O} 11-\mathrm{H} 29$ & $\mathrm{RY}^{*}(1) \mathrm{H} 16$ & 0.46 \\
\hline LP (1) 011 & $\mathrm{BD}^{*}(1) \mathrm{C} 1-\mathrm{H} 16$ & 1.00 \\
\hline LP (2) O11 & $\mathrm{BD}^{*}(1) \mathrm{C} 1-\mathrm{H} 16$ & 0.63 \\
\hline LP (2) O11 & $B D^{*}(2) C 3-C 4$ & 0.96 \\
\hline
\end{tabular}

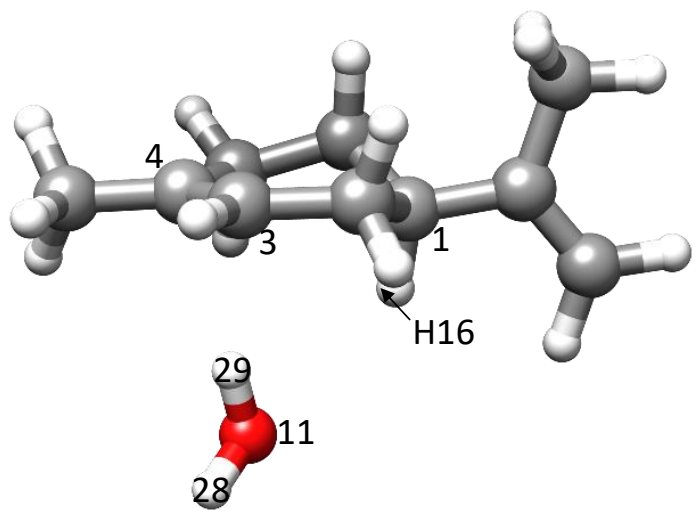

Table S11. Intermolecular stabilising energy contributions $\left(\geq 0.42 \mathrm{~kJ} \mathrm{~mol}^{-1}\right)$ for isomer EQC-3 $\beta$ of limonene- $\mathrm{H}_{2} \mathrm{O}$ from Natural Bond Orbital (NBO) analysis at the B3LYP-D3BJ/6-311++G(d,p) level of theory.

\begin{tabular}{|c|c|c|}
\hline \multicolumn{3}{|c|}{ From limonene to water } \\
\hline Donor & Acceptor & $\mathrm{kJ} / \mathrm{mol}$ \\
\hline $\mathrm{BD}(1) \mathrm{C} 3-\mathrm{C} 4$ & $\mathrm{RY} *(3) \mathrm{H} 29$ & 0.75 \\
\hline $\mathrm{BD}(2) \mathrm{C} 3-\mathrm{C} 4$ & $\mathrm{RY}^{*}(4) \mathrm{H} 29$ & 0.63 \\
\hline $\mathrm{BD}(2) \mathrm{C} 3-\mathrm{C} 4$ & $\mathrm{BD} *(1) \mathrm{O} 11-\mathrm{H} 29$ & 12.34 \\
\hline \multicolumn{3}{|c|}{ From water to limonene } \\
\hline Donor & Acceptor & $\mathrm{kJ} / \mathrm{mol}$ \\
\hline LP (1) O11 & $\mathrm{BD}^{*}(1) \mathrm{C} 6-\mathrm{H} 18$ & 0.42 \\
\hline LP (2) O11 & $\mathrm{BD}^{*}(1) \mathrm{C} 6-\mathrm{H} 18$ & 1.13 \\
\hline
\end{tabular}

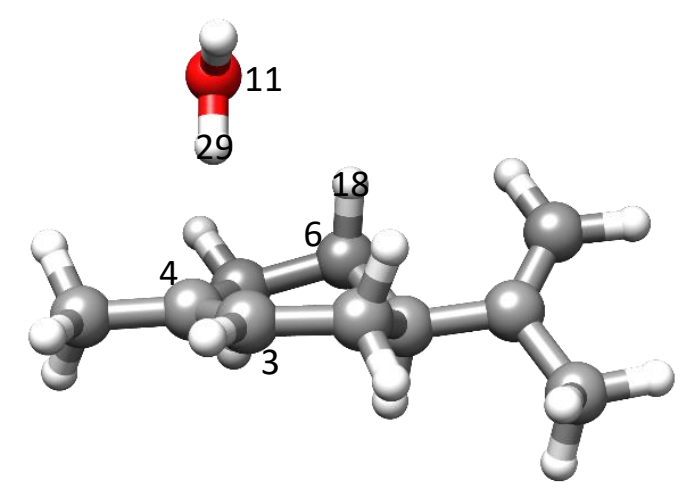


Table S12. Intermolecular stabilising energy contributions $\left(\geq 0.42 \mathrm{~kJ} \mathrm{~mol}^{-1}\right)$ for isomer EQC-4 of limonene- $\mathrm{H}_{2} \mathrm{O}$ from Natural Bond Orbital (NBO) analysis at the B3LYP-D3BJ/6-311++G(d,p) level of theory.

\begin{tabular}{|c|c|c|}
\hline \multicolumn{3}{|c|}{ From limonene to water } \\
\hline Donor & Acceptor & $\mathrm{kJ} / \mathrm{mol}$ \\
\hline $\mathrm{BD}(1) \mathrm{C2}-\mathrm{C} 3$ & $\mathrm{RY} *(3) \mathrm{H} 29$ & 0.84 \\
\hline $\mathrm{BD}(2) \mathrm{C} 2-\mathrm{C} 3$ & $R Y *(4) H 29$ & 0.71 \\
\hline $\mathrm{BD}(2) \mathrm{C2}-\mathrm{C} 3$ & $B D *(1) O 27-H 29$ & 13.39 \\
\hline $\mathrm{BD}(1) \mathrm{C} 6-\mathrm{H} 15$ & $B D^{*}(1) \mathrm{O} 27-\mathrm{H} 28$ & 0.50 \\
\hline \multicolumn{3}{|c|}{ From water to limonene } \\
\hline Donor & Acceptor & $\mathrm{kJ} / \mathrm{mol}$ \\
\hline $\mathrm{BD}$ (1) $\mathrm{O} 27-\mathrm{H} 28$ & $\mathrm{RY} *(1) \mathrm{H} 15$ & 1.72 \\
\hline LP (1) O27 & $B D^{*}(1) \mathrm{C} 6-\mathrm{H} 15$ & 1.17 \\
\hline LP (2) O27 & $B D^{*}(2) C 2-C 3$ & 1.13 \\
\hline
\end{tabular}

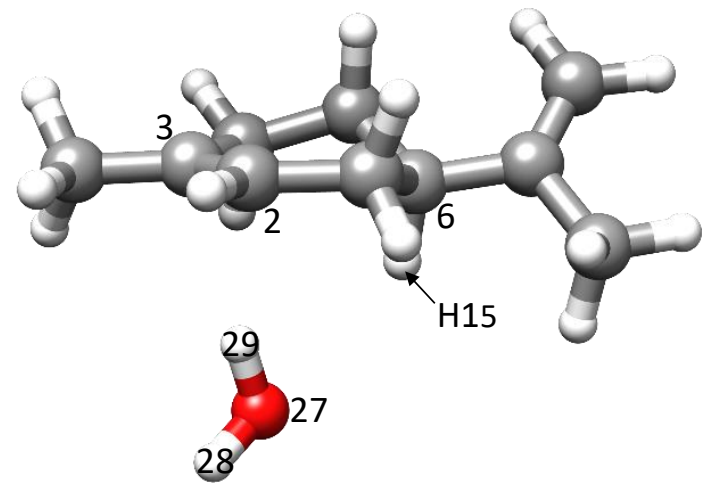

Table S13. Intermolecular stabilising energy contributions $\left(\geq 0.42 \mathrm{~kJ} \mathrm{~mol}^{-1}\right)$ for isomer EQC-2 of limonene- $\mathrm{H}_{2} \mathrm{O}$ from Natural Bond Orbital (NBO) analysis at the B3LYP-D3BJ/6-311++G(d,p) level of theory.

\begin{tabular}{|c|c|c|}
\hline \multicolumn{3}{|c|}{ From limonene to water } \\
\hline Donor & Acceptor & $\mathrm{kJ} / \mathrm{mol}$ \\
\hline $\mathrm{BD}(1) \mathrm{C} 5-\mathrm{H} 16$ & $\mathrm{RY}^{*}(2) \mathrm{O} 27$ & 0.46 \\
\hline $\mathrm{BD}(1) \mathrm{C} 9$ - C11 & $\mathrm{RY}^{*}(3) \mathrm{H} 29$ & 1.05 \\
\hline $\mathrm{BD}(2) \mathrm{C} 9-\mathrm{C} 11$ & $\mathrm{RY}^{*}(4) \mathrm{H} 29$ & 0.50 \\
\hline $\mathrm{BD}(2) \mathrm{C} 9-\mathrm{C} 11$ & $\mathrm{BD}^{*}(1) \mathrm{O} 27-\mathrm{H} 29$ & 12.97 \\
\hline \multicolumn{3}{|c|}{ From water to limonene } \\
\hline Donor & Acceptor & $\mathrm{kJ} / \mathrm{mol}$ \\
\hline LP (2) O27 & $\mathrm{BD} *(1) \mathrm{C} 5-\mathrm{H} 16$ & 0.42 \\
\hline LP (2) O27 & $\mathrm{BD} *(2) \mathrm{C} 9-\mathrm{C} 11$ & 1.09 \\
\hline
\end{tabular}

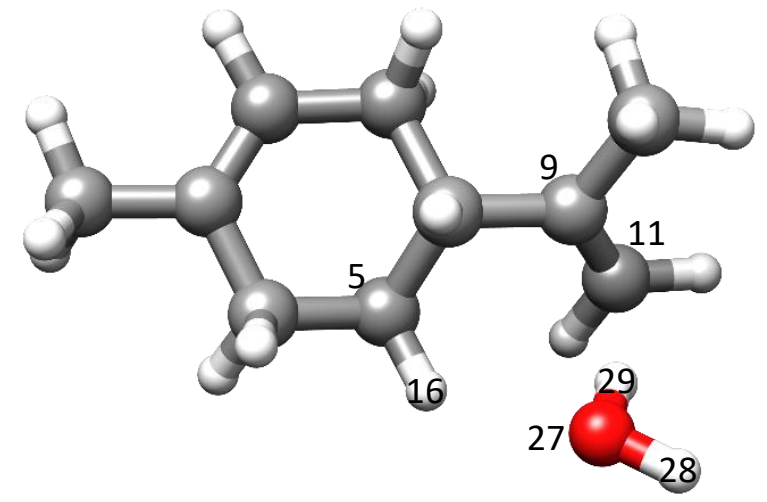

Table S14. Intermolecular stabilising energy contributions $\left(\geq 0.42 \mathrm{~kJ} \mathrm{~mol}^{-1}\right)$ for isomer EQa-4 of limonene- $\mathrm{H}_{2} \mathrm{O}$ from Natural Bond Orbital (NBO) analysis at the B3LYP-D3BJ/6-311++G(d,p) level of theory.

\begin{tabular}{|c|c|c|}
\hline \multicolumn{3}{|c|}{ From limonene to water } \\
\hline Donor & Acceptor & $\mathrm{kJ} / \mathrm{mol}$ \\
\hline $\mathrm{BD}(1) \mathrm{C} 2-\mathrm{C} 3$ & $\mathrm{RY}^{*}(3) \mathrm{H} 28$ & 0.79 \\
\hline $\mathrm{BD}(2) \mathrm{C} 2-\mathrm{C} 3$ & $\mathrm{RY}^{*}(4) \mathrm{H} 28$ & 0.71 \\
\hline $\mathrm{BD}(2) \mathrm{C} 2-\mathrm{C} 3$ & $\mathrm{BD}^{*}(1) \mathrm{O} 27-\mathrm{H} 28$ & 13.26 \\
\hline $\mathrm{BD}(1) \mathrm{C} 6-\mathrm{H} 15$ & $\mathrm{BD}^{*}(1) \mathrm{O} 27-\mathrm{H} 29$ & 0.42 \\
\hline \multicolumn{3}{|c|}{ From water to limonene } \\
\hline Donor & Acceptor & $\mathrm{kJ} / \mathrm{mol}$ \\
\hline $\mathrm{BD}(1) \mathrm{O} 27-\mathrm{H} 28$ & $\mathrm{RY} *(1) \mathrm{H} 15$ & 0.42 \\
\hline $\mathrm{BD}(1) \mathrm{O} 27-\mathrm{H} 28$ & $\mathrm{BD} *(1) \mathrm{C} 6-\mathrm{H} 15$ & 0.46 \\
\hline $\mathrm{BD}(1) \mathrm{O} 27-\mathrm{H} 29$ & $\mathrm{RY}^{*}(1) \mathrm{H} 15$ & 0.96 \\
\hline LP (1) O27 & $\mathrm{BD}^{*}(1) \mathrm{C} 6-\mathrm{H} 15$ & 1.00 \\
\hline LP (2) O27 & $B D^{*}(2) \mathrm{C} 2-\mathrm{C} 3$ & 0.92 \\
\hline LP (2) O27 & $\mathrm{BD} *(1) \mathrm{C} 6-\mathrm{H} 15$ & 0.96 \\
\hline
\end{tabular}

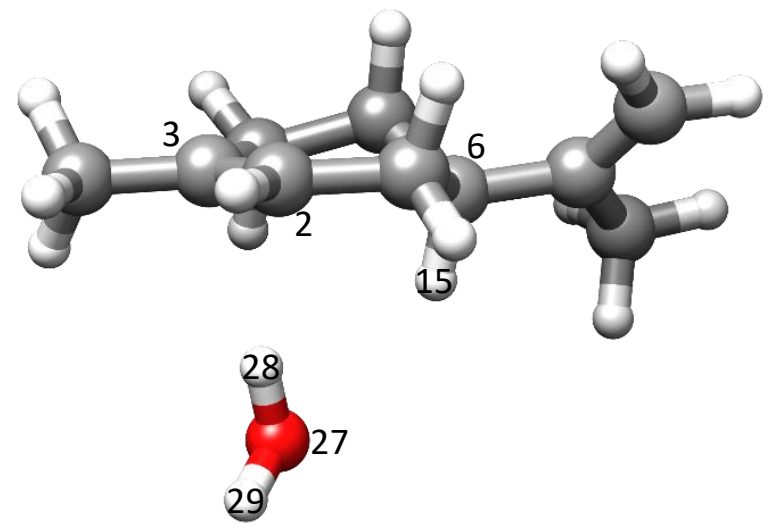


Table S15. Intermolecular stabilising energy contributions $\left(\geq 0.42 \mathrm{~kJ} \mathrm{~mol}^{-1}\right)$ for isomer AXa-2 $\alpha$ of limonene- $\mathrm{H}_{2} \mathrm{O}$ from Natural Bond Orbital (NBO) analysis at the B3LYP-D3BJ/6-311++G(d,p) level of theory.

\begin{tabular}{|c|c|c|}
\hline \multicolumn{3}{|c|}{ From limonene to water } \\
\hline Donor & Acceptor & $\mathrm{kJ} / \mathrm{mol}$ \\
\hline $\mathrm{BD}(1) \mathrm{C} 3-\mathrm{H} 12$ & $\mathrm{RY}^{*}(1) \mathrm{O} 11$ & 0.46 \\
\hline $\mathrm{BD}(1) \mathrm{C} 3-\mathrm{H} 12$ & $B D *(1) O 11-H 28$ & 0.59 \\
\hline $\mathrm{BD}(1) \mathrm{C} 8-\mathrm{C} 9$ & $\mathrm{RY}^{*}(3) \mathrm{H} 29$ & 0.84 \\
\hline $\mathrm{BD}(2) \mathrm{C} 8-\mathrm{C} 9$ & $B D *(1) O 11-H 29$ & 11.25 \\
\hline \multicolumn{3}{|c|}{ From water to limonene } \\
\hline Donor & Acceptor & $\mathrm{kJ} / \mathrm{mol}$ \\
\hline $\mathrm{BD}$ (1) $\mathrm{O} 11-\mathrm{H} 28$ & $\mathrm{RY}^{*}(1) \mathrm{H} 12$ & 1.63 \\
\hline $\mathrm{BD}$ (1) $\mathrm{O} 11-\mathrm{H} 28$ & $\mathrm{RY}^{*}(4) \mathrm{H} 12$ & 0.54 \\
\hline $\mathrm{BD}$ (1) $\mathrm{O} 11-\mathrm{H} 28$ & $\mathrm{RY}^{*}(1) \mathrm{H} 25$ & 0.54 \\
\hline $\mathrm{BD}$ (1) O11 - H29 & $\mathrm{RY}^{*}(1) \mathrm{H} 12$ & 0.54 \\
\hline $\mathrm{BD}$ (1) $011-\mathrm{H} 29$ & $B D *(1) C 3-H 12$ & 0.59 \\
\hline $\mathrm{BD}$ (1) O11 - H29 & $\mathrm{BD} *(2) \mathrm{C} 8-\mathrm{C} 9$ & 1.17 \\
\hline LP (1) 011 & $\mathrm{BD}^{*}(1) \mathrm{C} 7-\mathrm{H} 25$ & 1.84 \\
\hline LP (2) 011 & $B D *(1) C 3-H 12$ & 2.01 \\
\hline LP (2) 011 & $\mathrm{BD} *(2) \mathrm{C} 8-\mathrm{C} 9$ & 0.42 \\
\hline
\end{tabular}

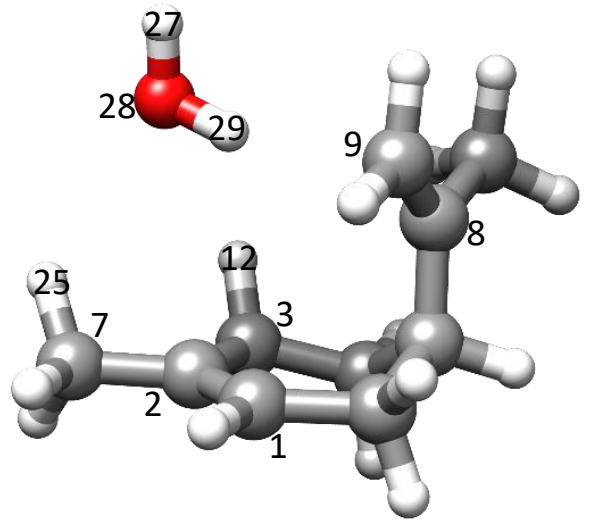

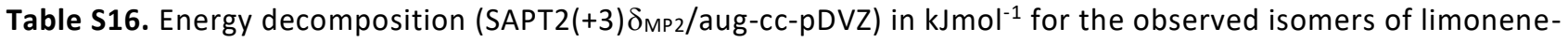
$\mathrm{H}_{2} \mathrm{O}$ on B3LYP-D3BJ/6-311++G(d,p) geometries.

\begin{tabular}{lrrrrr}
\hline \hline & $\Delta E_{\text {electrostatics }}$ & $\Delta \mathrm{E}_{\text {exchange }}$ & $\Delta \mathrm{E}_{\text {induction }}$ & $\Delta \mathrm{E}_{\text {dispersion }}$ & \multicolumn{1}{c}{$\Delta \mathrm{E}_{\text {total }}$} \\
\hline \hline AXa-2 $\alpha$ & -24.7 & 39.5 & -10.8 & -21.1 & -17.1 \\
EQA-3 & -24.3 & 38.0 & -12.0 & -18.9 & -17.2 \\
EQa-4 & -24.3 & 38.3 & -11.9 & -18.8 & -16.7 \\
EQC-4 & -23.7 & 37.4 & -11.8 & -18.6 & -16.8 \\
EQA-4 & -23.4 & 37.3 & -11.7 & -18.7 & -16.4 \\
EQC-2 & -22.2 & 32.9 & -10.5 & -16.2 & -16.0 \\
EQC-3 $\beta$ & -22.2 & 34.9 & -10.9 & -17.7 & -16.0 \\
\hline
\end{tabular}


Table S17. Cartesian coordinates of isomer EQA-3 from B3LYP-D3BJ/6-311++G(d,p) calculations.

\begin{tabular}{lrrr}
\hline \hline & $\mathrm{X}$ & $\mathrm{Y}$ & $\mathrm{Z}$ \\
\hline \hline $\mathrm{C}$ & -0.25018 & -0.13561 & -1.2855 \\
$\mathrm{C}$ & 1.23468 & -0.37596 & -1.2052 \\
$\mathrm{C}$ & 1.89517 & -0.67015 & -0.07749 \\
$\mathrm{C}$ & 1.18508 & -0.74789 & 1.25458 \\
$\mathrm{C}$ & -0.21732 & -0.13445 & 1.22573 \\
$\mathrm{C}$ & -0.99064 & -0.60344 & -0.01748 \\
$\mathrm{C}$ & 3.37143 & -0.95571 & -0.05914 \\
$\mathrm{H}$ & 1.13407 & -1.80240 & 1.55990 \\
$\mathrm{C}$ & -2.44123 & -0.16866 & -0.01568 \\
$\mathrm{C}$ & -2.73369 & 1.29557 & 0.19066 \\
$\mathrm{C}$ & -3.42121 & -1.05838 & -0.18844 \\
$\mathrm{H}$ & 1.78880 & -0.32634 & -2.14059 \\
$\mathrm{H}$ & -0.65805 & -0.65876 & -2.15673 \\
$\mathrm{H}$ & -0.43751 & 0.93054 & -1.46307 \\
$\mathrm{H}$ & -0.97590 & -1.69976 & -0.02127 \\
$\mathrm{H}$ & -0.76440 & -0.40703 & 2.13287 \\
$\mathrm{H}$ & -0.13067 & 0.95595 & 1.21612 \\
$\mathrm{H}$ & -4.46458 & -0.76141 & -0.20043 \\
$\mathrm{H}$ & -3.21023 & -2.11420 & -0.32235 \\
$\mathrm{H}$ & -2.18029 & 1.92346 & -0.51388 \\
$\mathrm{H}$ & -3.79819 & 1.50307 & 0.07082 \\
$\mathrm{H}$ & -2.43592 & 1.61793 & 1.19335 \\
$\mathrm{H}$ & 3.80675 & -0.91022 & -1.05954 \\
$\mathrm{H}$ & 3.56578 & -1.95288 & 0.35220 \\
$\mathrm{H}$ & 3.90087 & -0.24375 & 0.58419 \\
$\mathrm{H}$ & 1.79548 & -0.24861 & 2.01559 \\
$\mathrm{O}$ & 1.64083 & 2.59220 & 0.05061 \\
$\mathrm{H}$ & 2.38796 & 3.09163 & -0.29152 \\
$\mathrm{H}$ & 1.75672 & 1.69317 & -0.29280 \\
\hline & & &
\end{tabular}


Table S18. Cartesian coordinates of isomer EQA-4 from B3LYP-D3BJ/6-311++G(d,p) calculations.

\begin{tabular}{lrrr}
\hline \hline & $\mathrm{X}$ & $\mathrm{Y}$ & $\mathrm{Z}$ \\
\hline \hline $\mathrm{C}$ & -0.88249 & 0.13214 & -0.10471 \\
$\mathrm{C}$ & -0.25067 & -0.01008 & 1.29406 \\
$\mathrm{C}$ & 1.23402 & -0.25800 & 1.23483 \\
$\mathrm{C}$ & 1.89724 & -0.62871 & 0.13175 \\
$\mathrm{C}$ & 1.18086 & -0.85171 & -1.18026 \\
$\mathrm{C}$ & -0.33658 & -0.97716 & -1.01938 \\
$\mathrm{C}$ & 3.38587 & -0.84045 & 0.11828 \\
$\mathrm{C}$ & -2.39538 & 0.16882 & -0.04435 \\
$\mathrm{C}$ & -3.05838 & 1.30151 & -0.28691 \\
$\mathrm{C}$ & -3.11877 & -1.10934 & 0.29582 \\
$\mathrm{O}$ & 1.70193 & 2.58139 & -0.35237 \\
$\mathrm{H}$ & 1.42282 & -0.02213 & -1.85775 \\
$\mathrm{H}$ & 1.78783 & -0.13229 & 2.16331 \\
$\mathrm{H}$ & -0.45226 & 0.89713 & 1.87326 \\
$\mathrm{H}$ & -0.72835 & -0.82894 & 1.84770 \\
$\mathrm{H}$ & -0.54786 & 1.09101 & -0.51375 \\
$\mathrm{H}$ & -0.82341 & -0.93131 & -1.99779 \\
$\mathrm{H}$ & -0.57287 & -1.95621 & -0.58823 \\
$\mathrm{H}$ & -4.14119 & 1.34909 & -0.23744 \\
$\mathrm{H}$ & -2.53605 & 2.21696 & -0.54346 \\
$\mathrm{H}$ & -2.75438 & -1.54336 & 1.23220 \\
$\mathrm{H}$ & -4.19168 & -0.93831 & 0.39681 \\
$\mathrm{H}$ & -2.96874 & -1.86589 & -0.48113 \\
$\mathrm{H}$ & 3.83752 & -0.61140 & 1.08574 \\
$\mathrm{H}$ & 3.86059 & -0.21093 & -0.64289 \\
$\mathrm{H}$ & 3.62714 & -1.87837 & -0.13697 \\
$\mathrm{H}$ & 1.58518 & -1.75173 & -1.65798 \\
$\mathrm{H}$ & 2.29310 & 3.16914 & 0.12694 \\
$\mathrm{H}$ & 1.75297 & 1.73430 & 0.11567 \\
\hline
\end{tabular}

Table S19. Cartesian coordinates of isomer EQC-4 from B3LYP-D3BJ/6-311++G(d,p) calculations.

\begin{tabular}{lrrr}
\hline \hline & $\mathrm{X}$ & $\mathrm{Y}$ & $\mathrm{Z}$ \\
\hline \hline $\mathrm{C}$ & -0.25422 & 0.31464 & 1.21391 \\
$\mathrm{C}$ & 1.24386 & 0.15848 & 1.22632 \\
$\mathrm{C}$ & 1.94453 & -0.50548 & 0.2982 \\
$\mathrm{C}$ & 1.25682 & -1.19083 & -0.85897 \\
$\mathrm{C}$ & -0.24815 & -1.36783 & -0.64143 \\
$\mathrm{C}$ & -0.88470 & -0.06747 & -0.14373 \\
$\mathrm{C}$ & 3.44376 & -0.61125 & 0.33875 \\
$\mathrm{H}$ & 1.44131 & -0.61004 & -1.77264 \\
$\mathrm{C}$ & -2.39778 & -0.08043 & -0.05481 \\
$\mathrm{C}$ & -3.04084 & 1.25940 & 0.20210 \\
$\mathrm{C}$ & -3.13309 & -1.18586 & -0.19012 \\
$\mathrm{H}$ & 1.77425 & 0.61465 & 2.06003 \\
$\mathrm{H}$ & -0.50577 & 1.34888 & 1.46726 \\
$\mathrm{H}$ & -0.69627 & -0.30597 & 2.00539 \\
$\mathrm{H}$ & -0.61474 & 0.72618 & -0.85386 \\
$\mathrm{H}$ & -0.72199 & -1.68971 & -1.57218 \\
$\mathrm{H}$ & -0.41578 & -2.16084 & 0.09625 \\
$\mathrm{H}$ & -4.21478 & -1.14921 & -0.11923 \\
$\mathrm{H}$ & -2.69345 & -2.15747 & -0.37653 \\
$\mathrm{H}$ & -2.70843 & 1.99673 & -0.53635 \\
$\mathrm{H}$ & -4.12926 & 1.19293 & 0.16053 \\
$\mathrm{H}$ & -2.76462 & 1.65232 & 1.18586 \\
$\mathrm{H}$ & 3.86900 & -0.05236 & 1.17496 \\
$\mathrm{H}$ & 3.88545 & -0.23323 & -0.59049 \\
$\mathrm{H}$ & 3.75574 & -1.65759 & 0.43064 \\
$\mathrm{H}$ & 1.72962 & -2.16533 & -1.02738 \\
$\mathrm{O}$ & 1.45992 & 2.43317 & -1.09031 \\
$\mathrm{H}$ & 2.13416 & 3.09890 & -0.92613 \\
$\mathrm{H}$ & 1.61509 & 1.74556 & -0.42497 \\
\hline & & &
\end{tabular}


Table S20. Cartesian coordinates of isomer EQC-2 from B3LYP-D3BJ/6-311++G(d,p) calculations.

\begin{tabular}{lrrr}
\hline \hline & \multicolumn{1}{c}{$\mathrm{X}$} & $\mathrm{Y}$ & \multicolumn{1}{c}{$\mathrm{Z}$} \\
\hline $\mathrm{C}$ & -0.21369 & 0.81756 & 0.79539 \\
$\mathrm{C}$ & 0.38718 & -0.28623 & -0.07978 \\
$\mathrm{C}$ & -0.53614 & -1.52382 & -0.04159 \\
$\mathrm{C}$ & -1.99008 & -1.16283 & -0.19183 \\
$\mathrm{C}$ & -2.47744 & 0.07552 & -0.07913 \\
$\mathrm{C}$ & -1.58242 & 1.24390 & 0.25954 \\
$\mathrm{C}$ & 1.81656 & -0.66596 & 0.25087 \\
$\mathrm{C}$ & 2.50192 & -1.56219 & -0.74885 \\
$\mathrm{C}$ & -3.93245 & 0.39309 & -0.28399 \\
$\mathrm{C}$ & 2.45368 & -0.24366 & 1.34893 \\
$\mathrm{O}$ & 2.63029 & 2.21923 & -0.91333 \\
$\mathrm{H}$ & -1.45605 & 1.86956 & -0.63455 \\
$\mathrm{H}$ & -2.67081 & -1.98019 & -0.41872 \\
$\mathrm{H}$ & -0.25883 & -2.22412 & -0.83575 \\
$\mathrm{H}$ & -0.37726 & -2.06303 & 0.90283 \\
$\mathrm{H}$ & 0.39001 & 0.08239 & -1.11495 \\
$\mathrm{H}$ & 0.45223 & 1.68278 & 0.83096 \\
$\mathrm{H}$ & -0.32697 & 0.44625 & 1.82041 \\
$\mathrm{H}$ & 3.47367 & -0.55052 & 1.55545 \\
$\mathrm{H}$ & 1.97920 & 0.39511 & 2.08304 \\
$\mathrm{H}$ & 2.46180 & -1.12114 & -1.75020 \\
$\mathrm{H}$ & 3.54735 & -1.73087 & -0.48513 \\
$\mathrm{H}$ & 2.00751 & -2.53636 & -0.80917 \\
$\mathrm{H}$ & -4.50436 & -0.49161 & -0.57119 \\
$\mathrm{H}$ & -4.05863 & 1.15084 & -1.06619 \\
$\mathrm{H}$ & -4.37520 & 0.80830 & 0.62882 \\
$\mathrm{H}$ & -2.08694 & 1.88236 & 0.99438 \\
$\mathrm{H}$ & 3.50025 & 2.62899 & -0.91566 \\
$\mathrm{H}$ & 2.69793 & 1.48508 & -0.28518 \\
\hline & & &
\end{tabular}

Table S21. Cartesian coordinates of isomer EQa-4 from B3LYP-D3BJ/6-311++G(d,p) calculations.

\begin{tabular}{lrrr}
\hline \hline & $\mathrm{X}$ & $\mathrm{Y}$ & \multicolumn{1}{c}{$\mathrm{Z}$} \\
\hline \hline $\mathrm{C}$ & 0.18083 & -0.49605 & -1.29552 \\
$\mathrm{C}$ & -1.31329 & -0.58915 & -1.11985 \\
$\mathrm{C}$ & -1.93688 & -0.58750 & 0.06586 \\
$\mathrm{C}$ & -1.16254 & -0.50094 & 1.36111 \\
$\mathrm{C}$ & 0.33700 & -0.75259 & 1.18320 \\
$\mathrm{C}$ & 0.88405 & 0.01881 & -0.03499 \\
$\mathrm{C}$ & -3.43303 & -0.66714 & 0.19246 \\
$\mathrm{H}$ & -1.32984 & 0.49014 & 1.80376 \\
$\mathrm{C}$ & 2.39884 & -0.01546 & -0.08388 \\
$\mathrm{C}$ & 3.08305 & 0.92612 & 0.87456 \\
$\mathrm{C}$ & 3.10390 & -0.80490 & -0.89683 \\
$\mathrm{H}$ & -1.90820 & -0.68068 & -2.02648 \\
$\mathrm{H}$ & 0.41685 & 0.15576 & -2.14314 \\
$\mathrm{H}$ & 0.56263 & -1.48850 & -1.56545 \\
$\mathrm{H}$ & 0.59421 & 1.07000 & 0.09316 \\
$\mathrm{H}$ & 0.86912 & -0.47125 & 2.09485 \\
$\mathrm{H}$ & 0.52077 & -1.82097 & 1.02397 \\
$\mathrm{H}$ & 4.18830 & -0.79897 & -0.87721 \\
$\mathrm{H}$ & 2.63971 & -1.47758 & -1.60688 \\
$\mathrm{H}$ & 2.76396 & 0.74874 & 1.90663 \\
$\mathrm{H}$ & 4.16872 & 0.82459 & 0.83246 \\
$\mathrm{H}$ & 2.82273 & 1.96415 & 0.64024 \\
$\mathrm{H}$ & -3.92319 & -0.68131 & -0.78328 \\
$\mathrm{H}$ & -3.82053 & 0.18536 & 0.76175 \\
$\mathrm{H}$ & -3.72842 & -1.57037 & 0.73790 \\
$\mathrm{H}$ & -1.58282 & -1.21883 & 2.07536 \\
$\mathrm{O}$ & -1.54580 & 2.60376 & -0.35785 \\
$\mathrm{H}$ & -1.66740 & 1.66771 & -0.57819 \\
$\mathrm{H}$ & -2.07172 & 3.08474 & -1.00343 \\
\hline & & &
\end{tabular}


Table S22. Cartesian coordinates of isomer EQC-3 $\beta$ from B3LYP-D3BJ/6-311++G(d,p) calculations.

\begin{tabular}{llll}
\hline \hline & $\mathrm{X}$ & $\mathrm{Y}$ & $\mathrm{Z}$ \\
\hline \hline $\mathrm{C}$ & -1.03364 & -0.39523 & 0.26281 \\
$\mathrm{C}$ & -0.33935 & -0.5476 & -1.10883 \\
$\mathrm{C}$ & 1.13105 & -0.85042 & -0.98193 \\
$\mathrm{C}$ & 1.83662 & -0.71556 & 0.14815 \\
$\mathrm{C}$ & 1.20103 & -0.18217 & 1.41035 \\
$\mathrm{C}$ & -0.15899 & 0.47732 & 1.16757 \\
$\mathrm{C}$ & 3.29146 & -1.08285 & 0.24147 \\
$\mathrm{C}$ & -2.46488 & 0.07577 & 0.09762 \\
$\mathrm{C}$ & -2.87922 & 1.29507 & 0.44728 \\
$\mathrm{C}$ & -3.41314 & -0.92945 & -0.50582 \\
$\mathrm{O}$ & 2.15973 & 2.32033 & -0.83957 \\
$\mathrm{H}$ & 1.10347 & -1.00921 & 2.1275 \\
$\mathrm{H}$ & 1.63026 & -1.22975 & -1.87146 \\
$\mathrm{H}$ & -0.81578 & -1.3437 & -1.68935 \\
$\mathrm{H}$ & -0.48944 & 0.37456 & -1.68691 \\
$\mathrm{H}$ & -1.07412 & -1.39916 & 0.71067 \\
$\mathrm{H}$ & -0.66088 & 0.65851 & 2.12144 \\
$\mathrm{H}$ & 0.00032 & 1.45149 & 0.6949 \\
$\mathrm{H}$ & -3.9109 & 1.59715 & 0.30264 \\
$\mathrm{H}$ & -2.21555 & 2.0313 & 0.88259 \\
$\mathrm{H}$ & -3.42859 & -1.85144 & 0.08618 \\
$\mathrm{H}$ & -4.43034 & -0.53775 & -0.55743 \\
$\mathrm{H}$ & -3.10804 & -1.21056 & -1.51903 \\
$\mathrm{H}$ & 3.66677 & -1.49897 & -0.69565 \\
$\mathrm{H}$ & 3.45115 & -1.82353 & 1.03331 \\
$\mathrm{H}$ & 3.8972 & -0.20756 & 0.50007 \\
$\mathrm{H}$ & 1.88475 & 0.5358 & 1.87653 \\
$\mathrm{H}$ & 2.2032 & 2.66014 & -1.73797 \\
$\mathrm{H}$ & 1.99297 & 1.37072 & -0.93358 \\
\hline & & &
\end{tabular}

Table S23. Cartesian coordinates of isomer AXa-2 $\alpha$ from B3LYP-D3BJ/6-311++G(d,p) calculations.

\begin{tabular}{lrrr}
\hline & $\mathrm{X}$ & $\mathrm{Y}$ & $\mathrm{Z}$ \\
\hline $\mathrm{C}$ & 1.24512 & -1.03017 & -1.01217 \\
$\mathrm{C}$ & 1.68915 & -0.36487 & 0.05848 \\
$\mathrm{C}$ & 0.86968 & -0.27459 & 1.32547 \\
$\mathrm{C}$ & -0.31973 & -1.23785 & 1.34325 \\
$\mathrm{C}$ & -1.06801 & -1.23676 & -0.00759 \\
$\mathrm{C}$ & -0.09262 & -1.72218 & -1.08800 \\
$\mathrm{C}$ & 3.00355 & 0.36214 & 0.07491 \\
$\mathrm{C}$ & -1.73451 & 0.09878 & -0.29844 \\
$\mathrm{C}$ & -1.50241 & 0.82797 & -1.39529 \\
$\mathrm{C}$ & -2.73669 & 0.56031 & 0.72968 \\
$\mathrm{O}$ & 0.59287 & 2.84896 & 0.04403 \\
$\mathrm{H}$ & 0.53421 & 0.76157 & 1.45674 \\
$\mathrm{H}$ & 1.87423 & -1.08159 & -1.89775 \\
$\mathrm{H}$ & 0.04548 & -2.80511 & -0.97070 \\
$\mathrm{H}$ & -0.52854 & -1.59345 & -2.08334 \\
$\mathrm{H}$ & -1.88774 & -1.96340 & 0.07031 \\
$\mathrm{H}$ & -0.99320 & -0.99692 & 2.16883 \\
$\mathrm{H}$ & 0.03988 & -2.25655 & 1.52100 \\
$\mathrm{H}$ & -2.05640 & 1.74325 & -1.57865 \\
$\mathrm{H}$ & -0.78558 & 0.53411 & -2.15100 \\
$\mathrm{H}$ & -3.45258 & -0.23523 & 0.96232 \\
$\mathrm{H}$ & -3.29045 & 1.43247 & 0.37936 \\
$\mathrm{H}$ & -2.24499 & 0.83007 & 1.66905 \\
$\mathrm{H}$ & 3.53643 & 0.25137 & -0.87196 \\
$\mathrm{H}$ & 2.83814 & 1.42923 & 0.25682 \\
$\mathrm{H}$ & 3.64846 & -0.01015 & 0.87930 \\
$\mathrm{H}$ & 1.51969 & -0.47552 & 2.18532 \\
$\mathrm{H}$ & 0.25670 & 3.63249 & -0.40068 \\
$\mathrm{H}$ & 0.08214 & 2.11494 & -0.32894 \\
\hline & & &
\end{tabular}


Figure S3. Interconversion barrier between isomers EQC-3 $\alpha$ and EQC-3 $\beta$ of limonene- $\mathrm{H}_{2} \mathrm{O}$ at the B3LYP-D3BJ/6$311++G(d, p)$ level of theory.

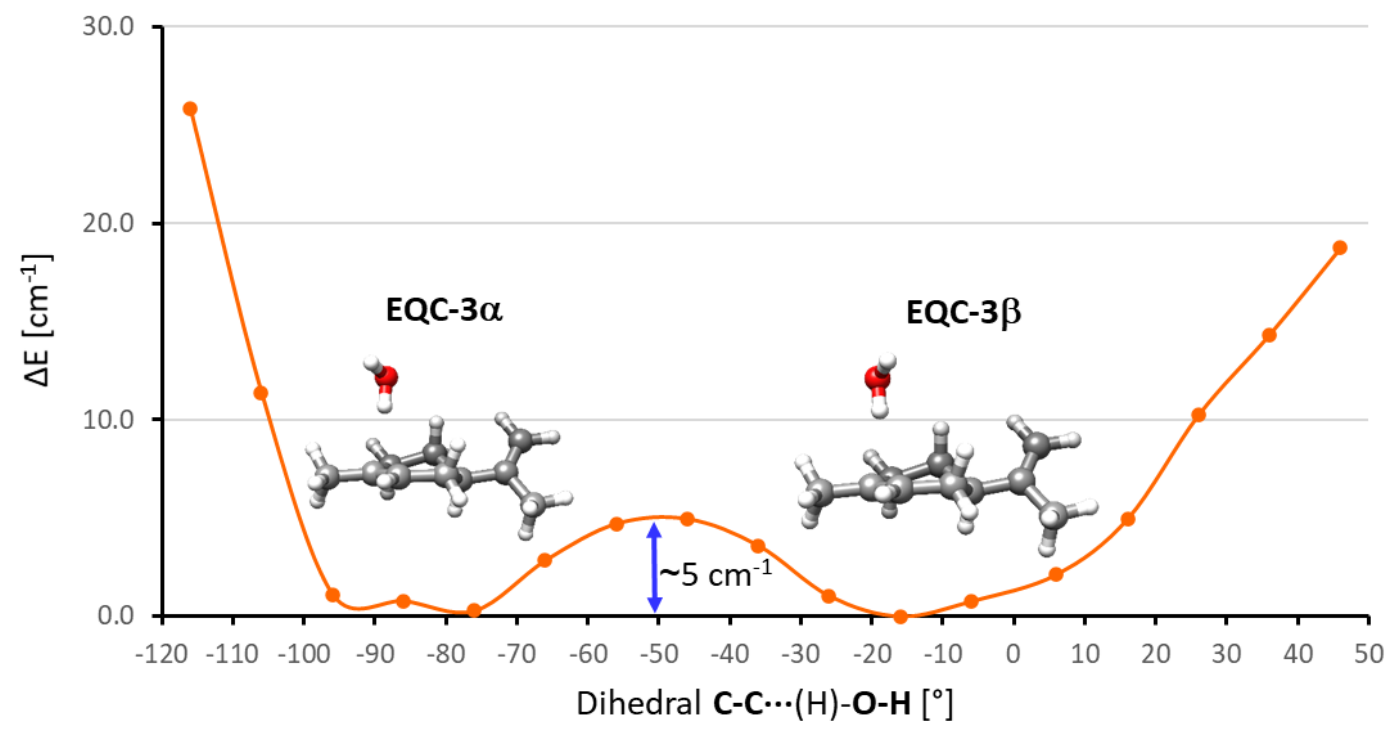

Figure S4. Interconversion barrier between isomers EQA-2 and EQC-2 of limonene- $\mathrm{H}_{2} \mathrm{O}$ at the B3LYP-D3BJ/6$311++G(d, p)$ level of theory.

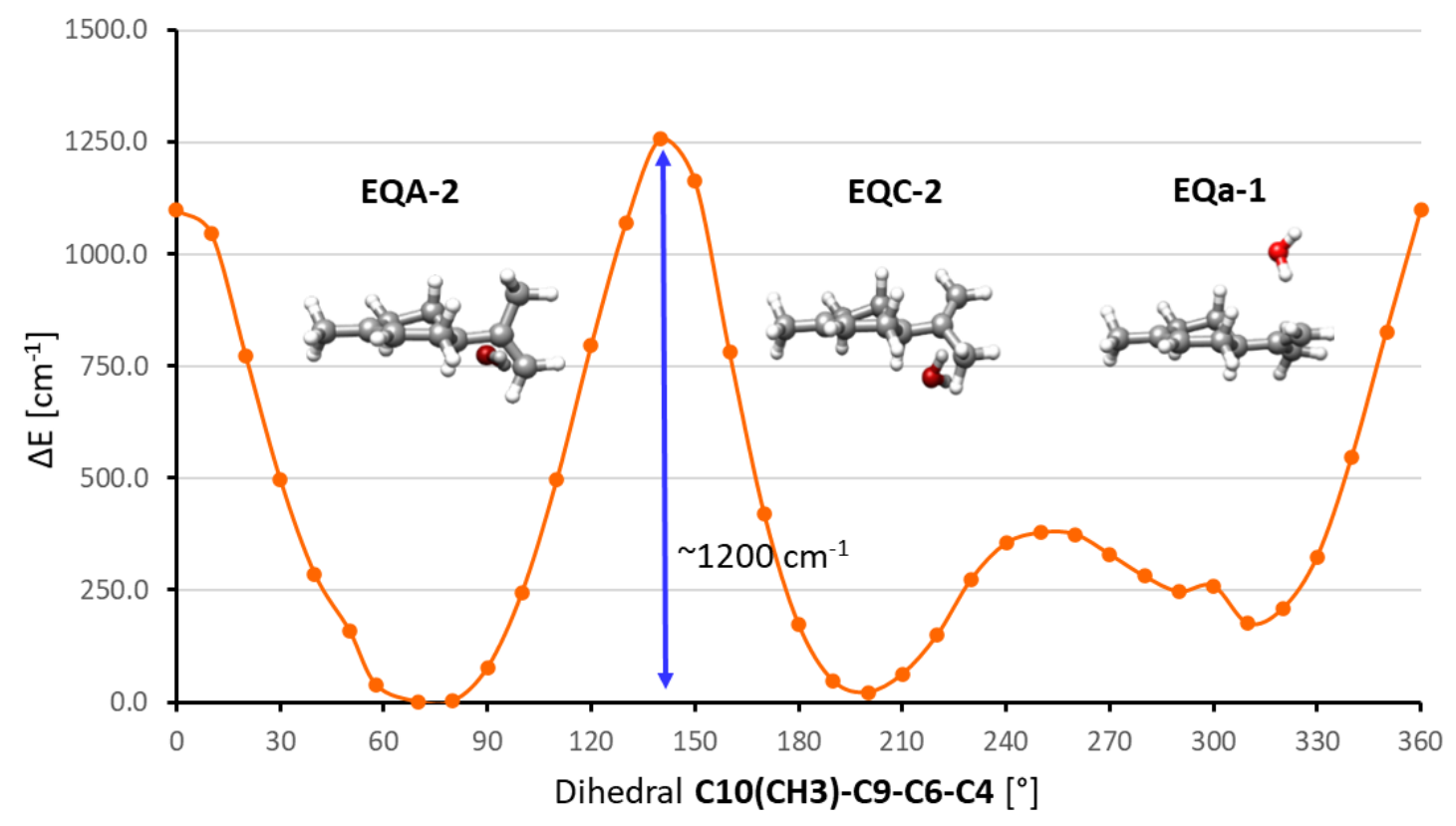


Figure S5. Interconversion barriers between isomers EQA-3, EQC-3 and EQa-3 of limonene- $\mathrm{H}_{2} \mathrm{O}$ at the B3LYP$D 3 B J / 6-311++G(d, p)$ level of theory.

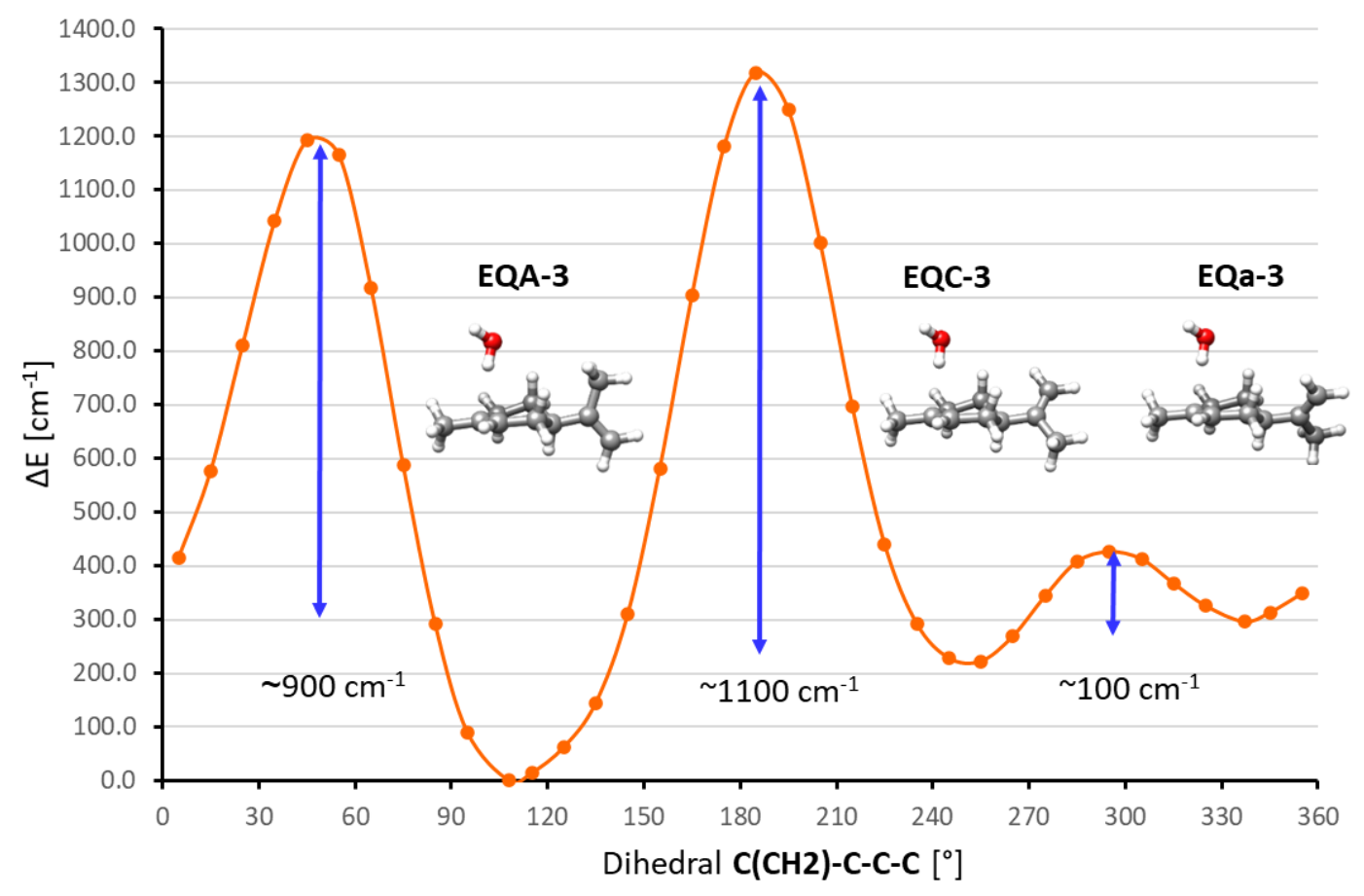

Figure S6. Interconversion barriers between isomers EQA-4, EQC-4 and EQa-4 of limonene- $\mathrm{H}_{2} \mathrm{O}$ at the B3LYP$D 3 B J / 6-311++G(d, p)$ level of theory.

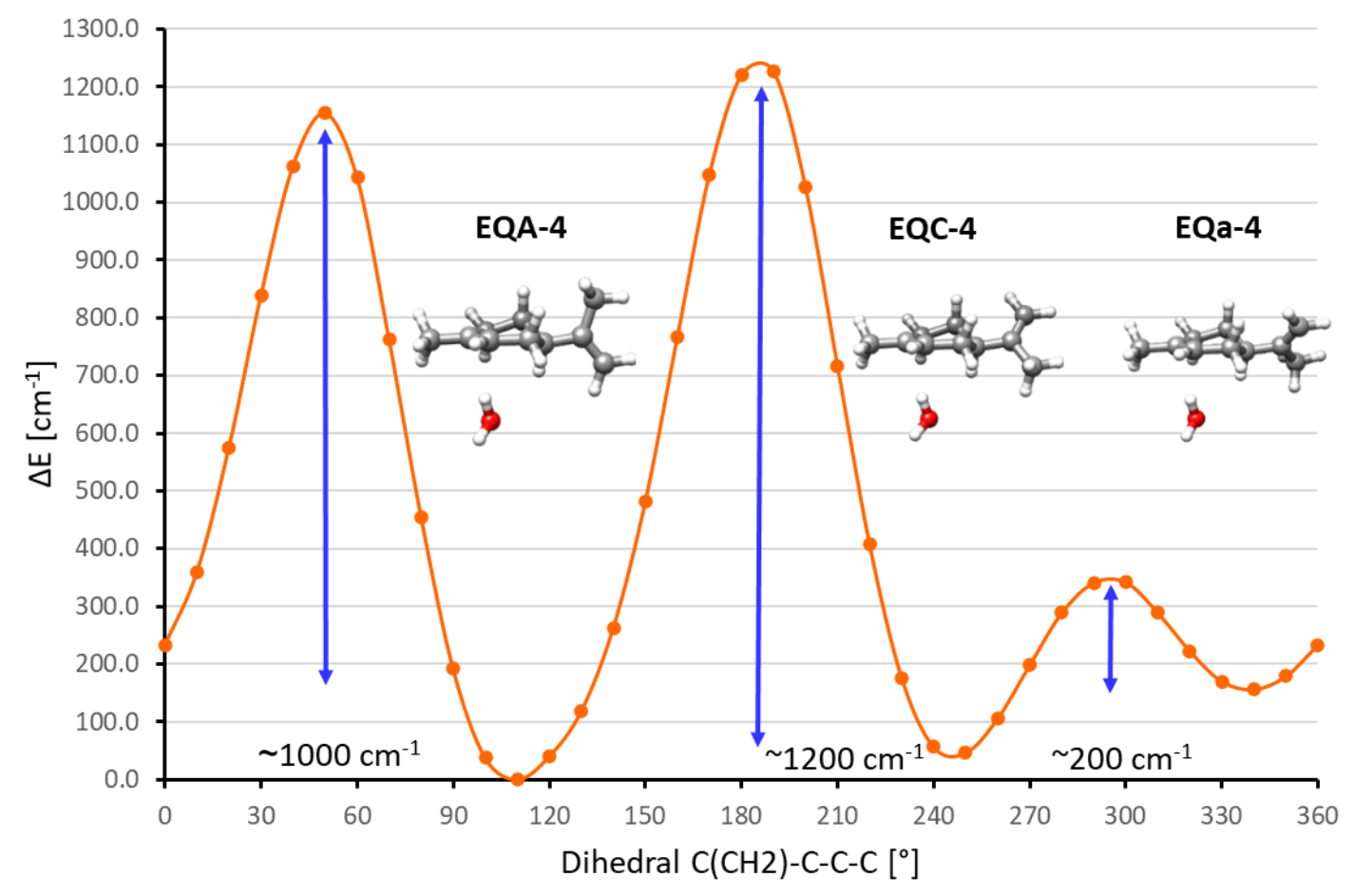


Figure S7. Interconversion barriers between isomers AXa-4 and EQa-4 of limonene- ${ }_{2} \mathrm{O}$ at the B3LYP-D3BJ/6$311++G(d, p)$ level of theory.

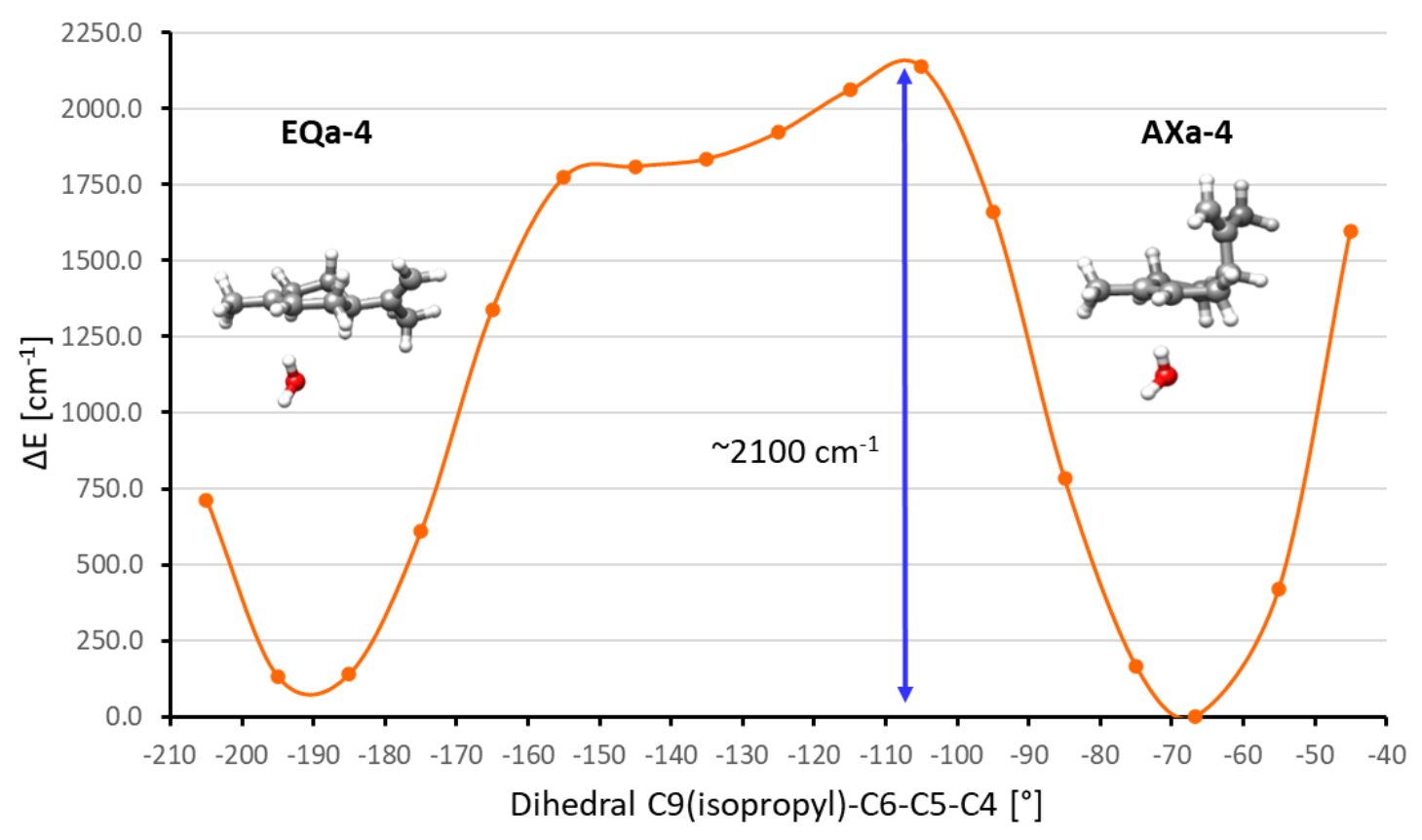

Figure S8. Interconversion barriers between isomers AXa-3 and EQC-3 of limonene- ${ }_{2} \mathrm{O}$ at the B3LYP-D3BJ/6$311++G(d, p)$ level of theory.

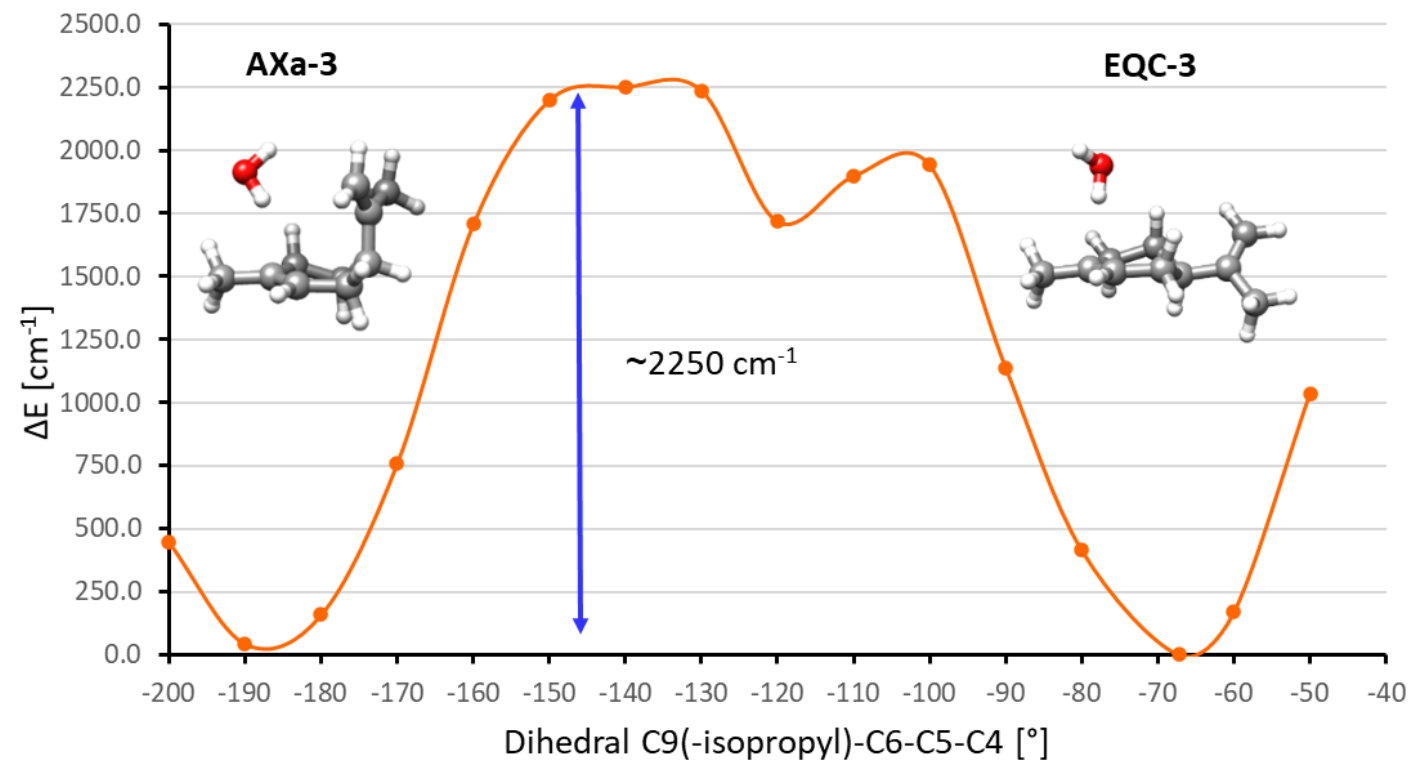


Figure S9. Interconversion barriers between isomers $\mathbf{A X a - 3 ,} \mathbf{A X a - 2} \boldsymbol{\alpha}$ and $\mathbf{A X a - 2} \boldsymbol{\beta}$ of limonene- $\mathrm{H}_{2} \mathrm{O}$ at the $\mathbf{M P 2} / 6-$ $311++G(d, p)$ level of theory.

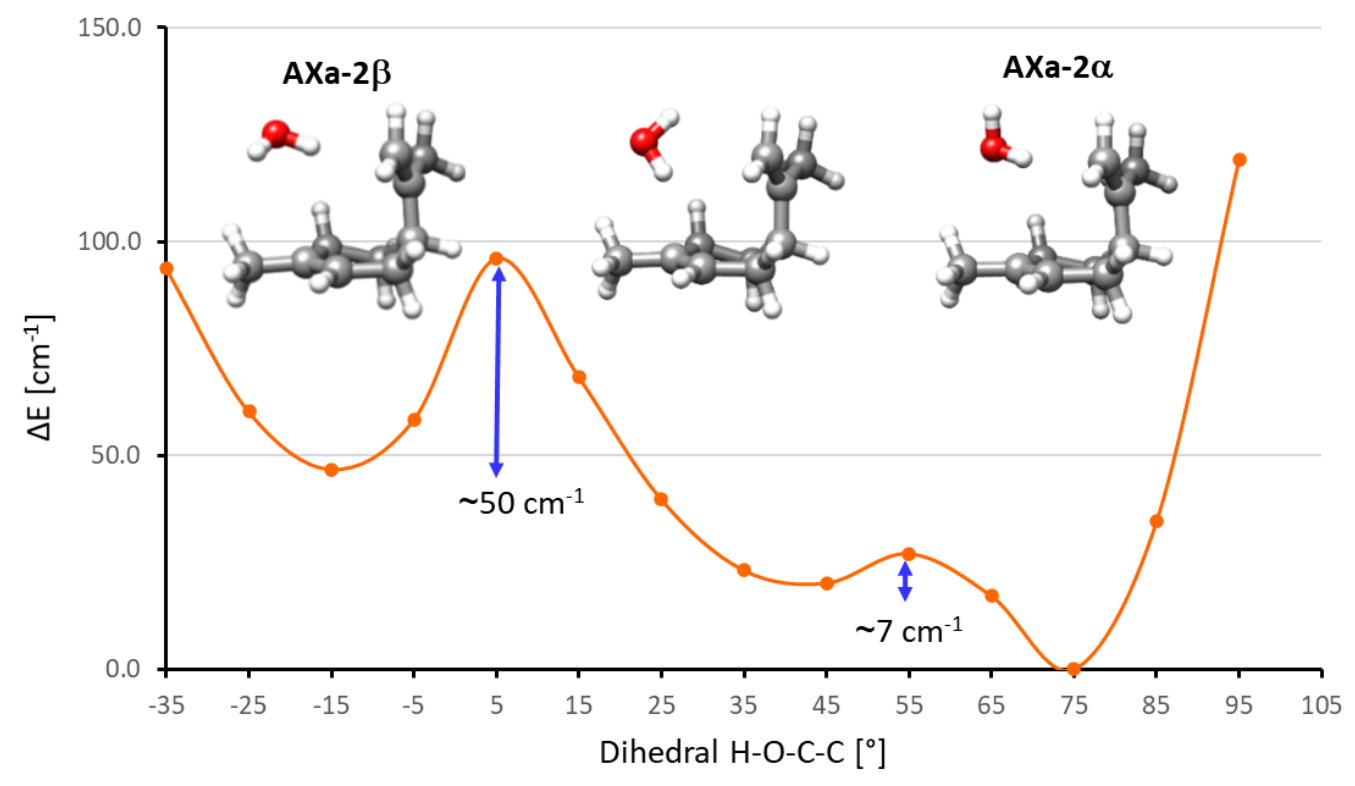

Figure S10. Interconversion barriers between isomers $\mathbf{A X a - 3 ,} \mathbf{A X a - 2} \boldsymbol{\alpha}$ and $\mathbf{A X a - 2} \boldsymbol{\beta}$ of limonene- $\mathrm{H}_{2} \mathrm{O}$ at the $\mathbf{B} \mathbf{3} \mathbf{L} \mathbf{P}$ D3BJ/6-311++G(d,p) level of theory.

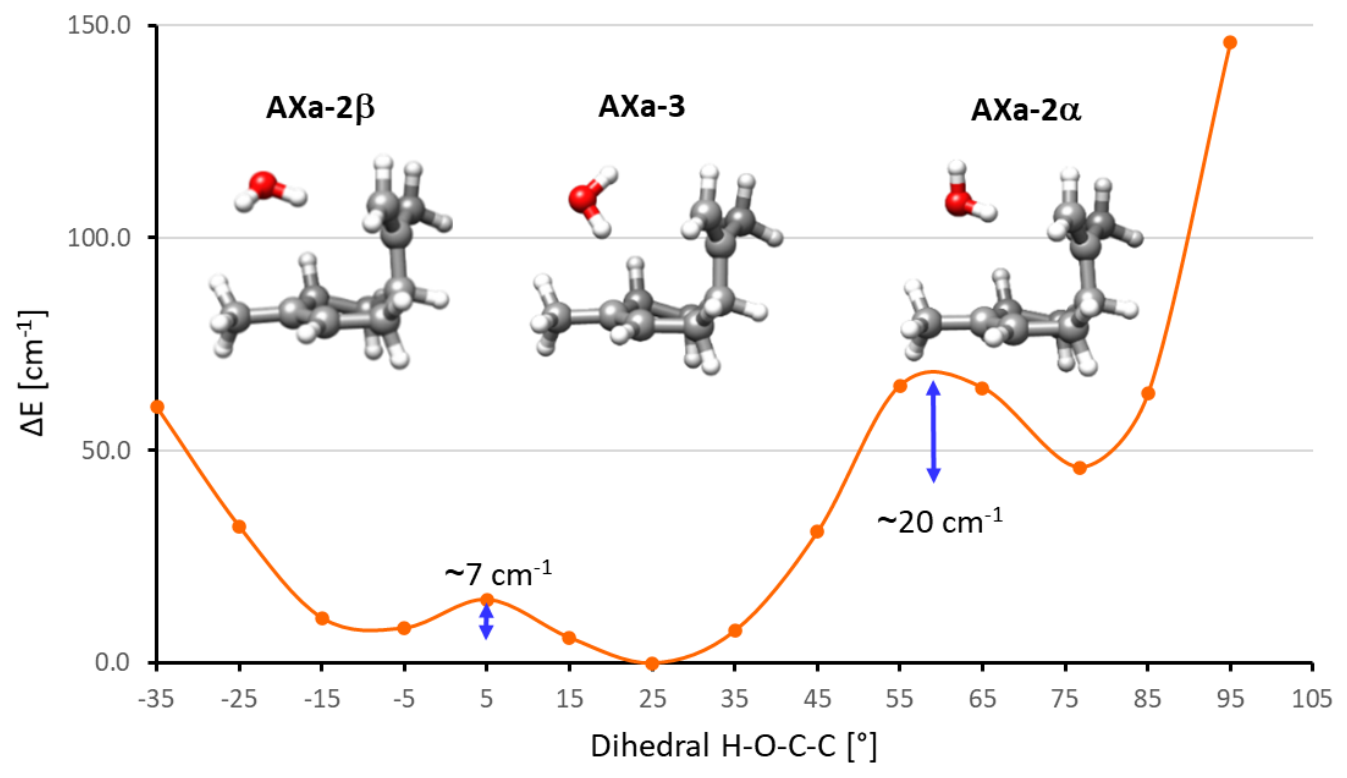


Figure S11. 3D plots of the molecular electrostatic potential (MEP) mapped on the $0.001 E_{h}$ isodensity surface calculated at the MP2/6-311++G(d,p) level of theory for the observed conformers of limonene. Areas of high electronegativity have been labelled with their values in $\mathrm{kJmol}^{-1}$. For the exocyclic double bond where one side of the surface is visible, the first value given corresponds to the visible surface and the second for the surface out of view.

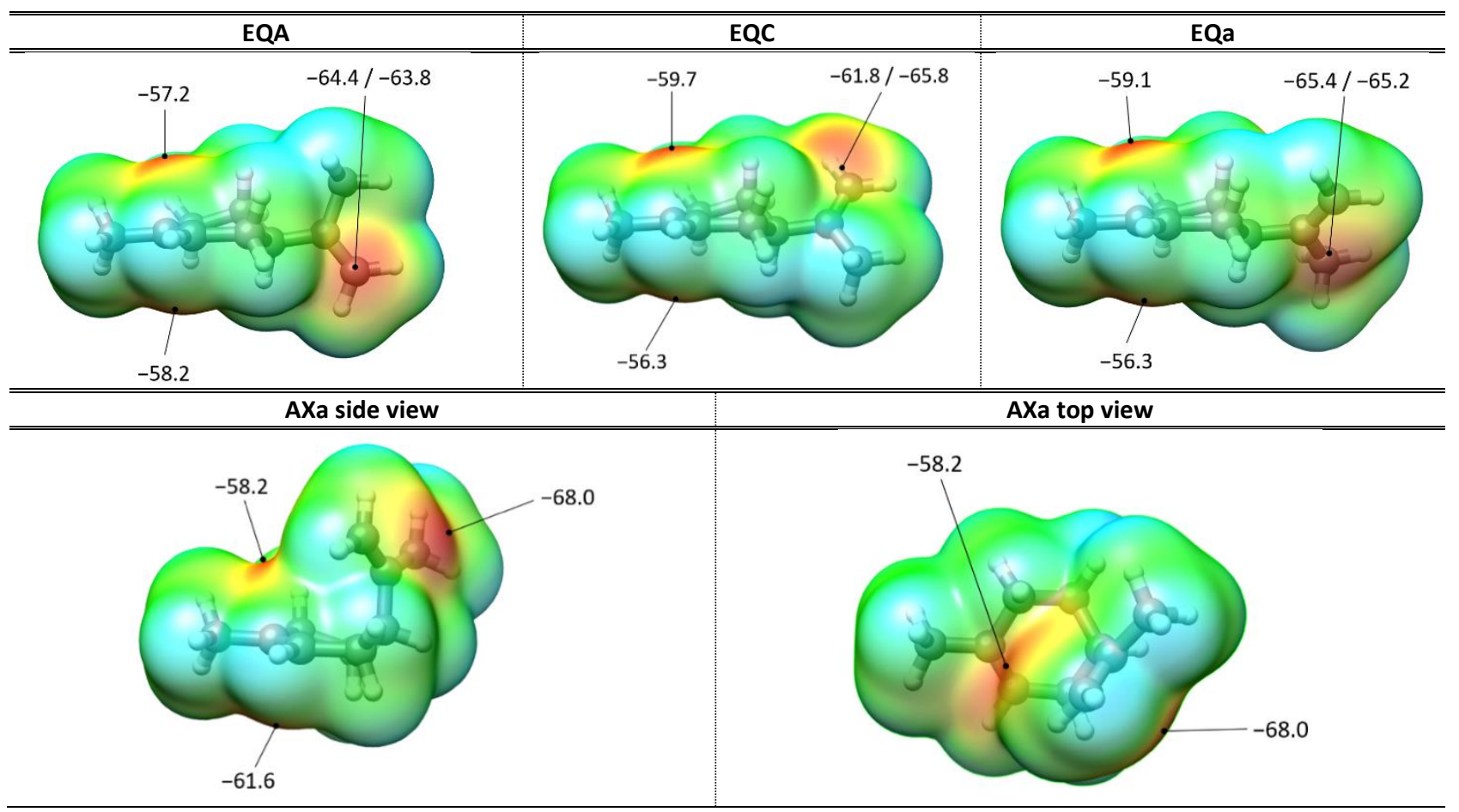


Figure S12. Plots of the reduced density gradient (RDG) versus $\operatorname{sign}(\lambda 2) \rho$ for the observed isomers of limonene- $\mathrm{H}_{2} \mathrm{O}$.

\section{EQA-3}

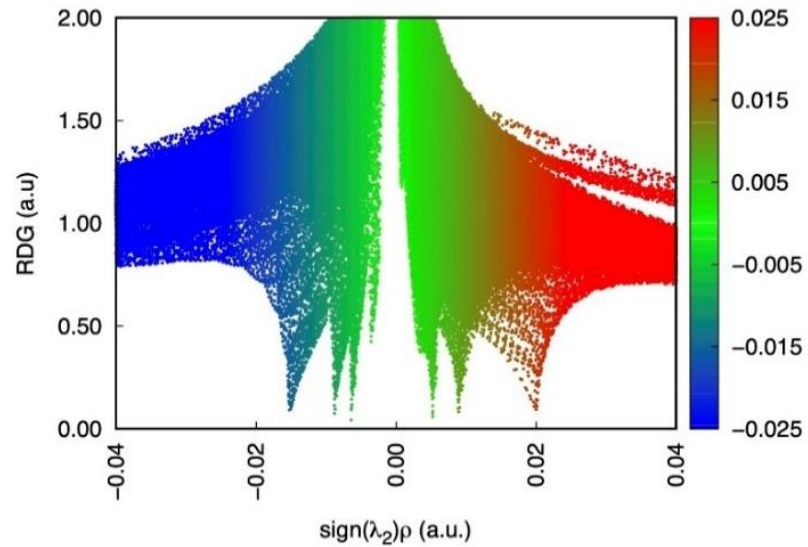

EQC-4

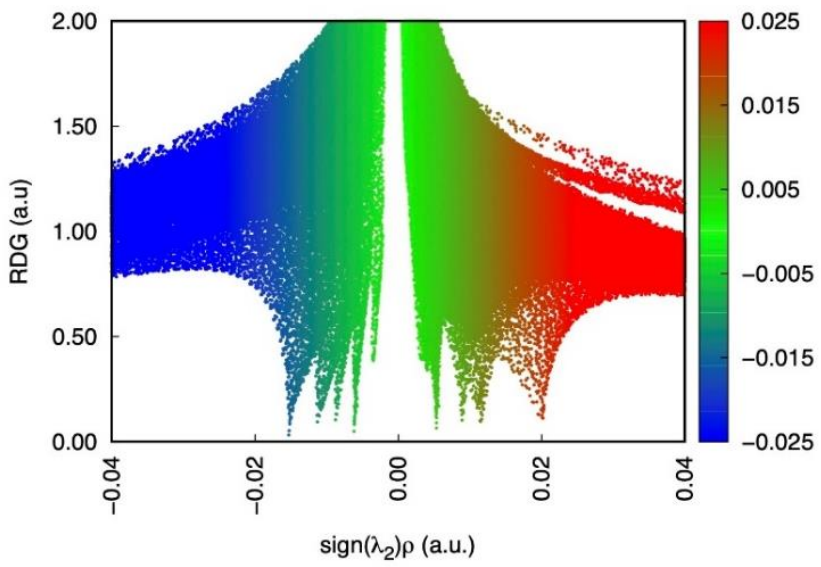

EQa-4

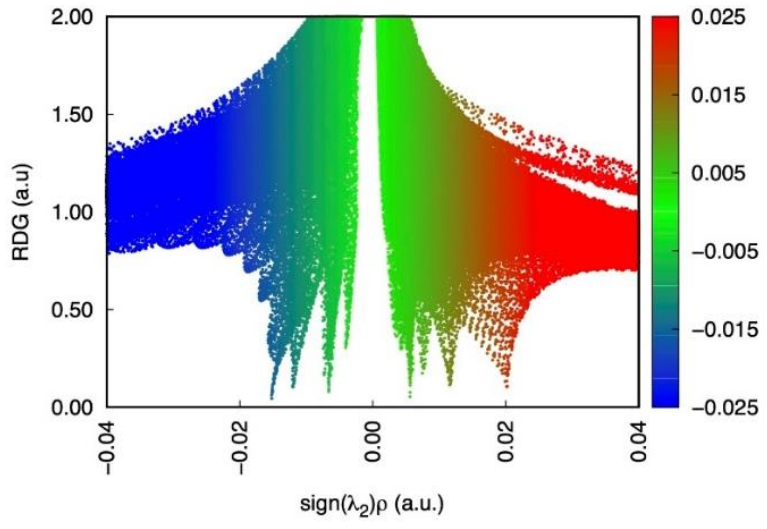

\section{EQA-4}

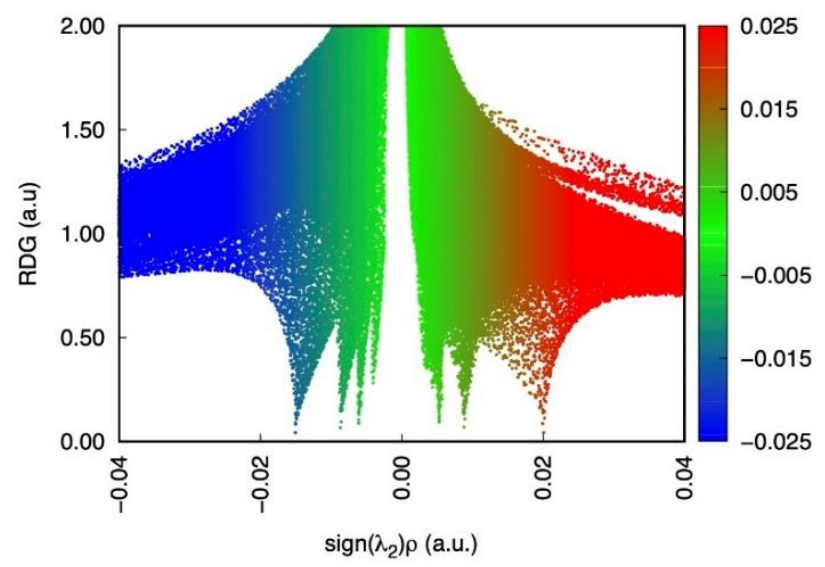

EQC-2

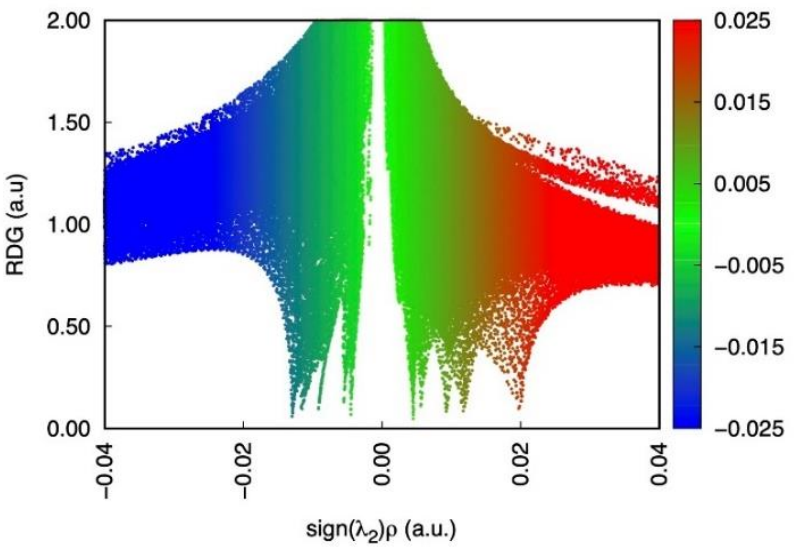

EQC-3 $\beta$

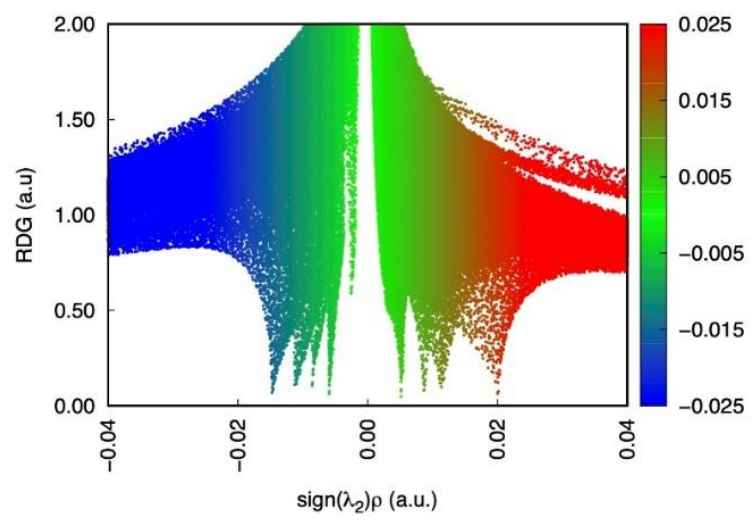


AXa-2 $\alpha$

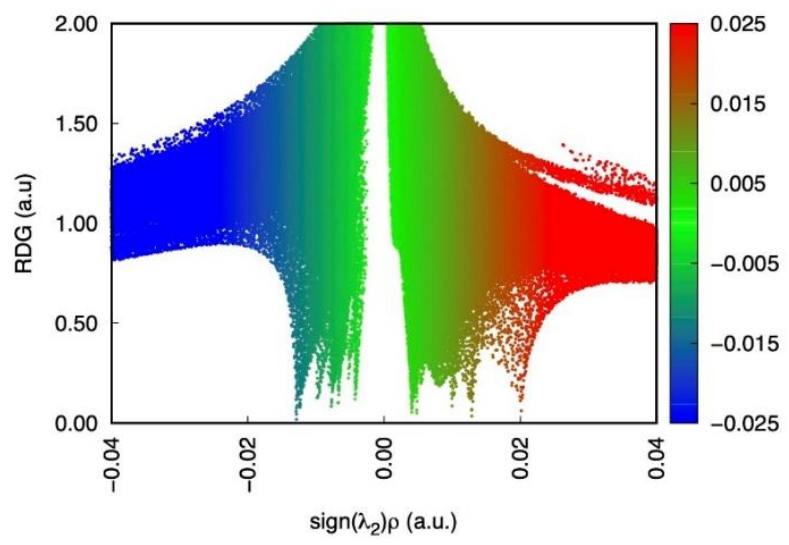


Frequencies of measured transitions and their residuals.

Table S24. Measured frequencies ( $\left.\nu_{\mathrm{obs}}\right)$ and residuals $\left(\nu_{\mathrm{obs}}-v_{\mathrm{cal}}\right)$ of the rotational transitions of the parent species of isomer EQA-3 of limonene- $\mathrm{H}_{2} \mathrm{O}$.

\begin{tabular}{|c|c|c|c|c|c|c|}
\hline$J^{\prime}$ & $K_{-1}^{\prime}$ & $K_{+1}^{\prime}$ & $J^{\prime \prime} K^{\prime \prime}$ & $K_{+1}^{\prime \prime}$ & $v_{\mathrm{obs}} / \mathrm{MHz}$ & $v_{\mathrm{obs}}-v_{\mathrm{calc}} / \mathrm{MHz}$ \\
\hline 2 & 1 & 2 & 11 & 1 & 2295.1047 & -0.0017 \\
\hline 2 & 0 & 2 & 1 & 1 & 2370.1457 & 0.0003 \\
\hline 2 & 1 & 1 & 1 & 0 & 2454.8661 & -0.0033 \\
\hline 3 & 1 & 3 & 21 & 2 & 3439.7102 & -0.0012 \\
\hline 3 & 0 & 3 & 2 & 2 & 3543.2096 & 0.0003 \\
\hline 3 & 1 & 2 & 2 & 1 & 3679.1710 & 0.0023 \\
\hline 4 & 1 & 4 & 3 & 3 & 4581.0285 & -0.0017 \\
\hline 4 & 0 & 4 & 3 & 3 & 4702.5269 & 0.0005 \\
\hline 4 & 1 & 3 & 3 & 2 & 4899.4423 & -0.0017 \\
\hline 5 & 1 & 5 & 4 & 4 & 5718.3658 & -0.0021 \\
\hline 5 & 0 & 5 & 4 & 4 & 5845.4472 & -0.0003 \\
\hline 5 & 1 & 4 & 4 & 3 & 6113.9146 & 0.0009 \\
\hline 6 & 1 & 6 & 5 & 5 & 6851.3277 & -0.0008 \\
\hline 6 & 0 & 6 & 5 & 5 & 6971.9312 & -0.0031 \\
\hline 6 & 1 & 5 & 5 & 4 & 7320.4319 & 0.0016 \\
\hline 7 & 1 & 7 & 6 & 6 & 7979.8285 & 0.0002 \\
\hline 3 & 2 & 2 & 2 & 1 & 3562.4518 & 0.0019 \\
\hline 3 & 2 & 1 & 2 & 0 & 3581.7186 & -0.0060 \\
\hline 4 & 2 & 3 & 3 & 2 & 4746.1469 & -0.0003 \\
\hline 4 & 2 & 2 & 3 & 1 & 4793.5800 & -0.0017 \\
\hline 5 & 2 & 4 & 4 & 3 & 5926.6148 & 0.0022 \\
\hline 5 & 2 & 3 & 4 & 2 & 6018.5508 & -0.0073 \\
\hline 6 & 2 & 5 & 5 & 4 & 7103.0725 & 0.0007 \\
\hline 6 & 2 & 4 & 5 & 3 & 7255.8230 & -0.0055 \\
\hline 4 & 3 & 2 & 3 & 1 & 4759.0379 & 0.0066 \\
\hline 5 & 3 & 3 & 4 & 2 & 5952.1325 & 0.0099 \\
\hline 5 & 3 & 2 & 4 & 1 & 5955.9694 & 0.0052 \\
\hline 6 & 3 & 3 & 53 & 2 & 7156.6742 & -0.0084 \\
\hline 6 & 3 & 4 & 5 & 3 & 7146.5324 & -0.0008 \\
\hline 3 & 0 & 3 & 2 & 2 & 2672.2550 & 0.0017 \\
\hline 4 & 0 & 4 & 3 & 3 & 3935.0728 & 0.0045 \\
\hline 5 & 0 & 5 & 4 & 4 & 5199.4898 & 0.0042 \\
\hline 6 & 0 & 6 & 51 & 5 & 6453.0555 & 0.0035 \\
\hline 7 & 0 & 7 & 6 & 6 & 7686.6229 & 0.0082 \\
\hline 2 & 1 & 2 & 1 & 1 & 3241.1040 & 0.0025 \\
\hline 3 & 1 & 3 & 2 & 2 & 4310.6674 & 0.0000 \\
\hline 4 & 1 & 4 & 3 & 3 & 5348.4857 & -0.0026 \\
\hline 5 & 1 & 5 & 4 & 4 & 6364.3276 & -0.0021 \\
\hline 6 & 1 & 6 & 5 & 5 & 7370.2009 & -0.0098 \\
\hline 2 & 1 & 1 & 1 & 1 & 3480.7494 & 0.0029 \\
\hline 3 & 1 & 2 & 2 & 2 & 4789.7730 & 0.0032 \\
\hline 4 & 1 & 3 & 3 & 3 & 6146.0076 & 0.0032 \\
\hline 5 & 1 & 4 & 4 & 4 & 7557.3961 & 0.0043 \\
\hline 3 & 2 & 1 & 2 & 1 & 6424.5390 & -0.0025 \\
\hline 4 & 2 & 2 & 3 & 2 & 7538.9486 & -0.0059 \\
\hline 2 & 2 & 1 & 1 & 1 & 5372.7161 & -0.0041 \\
\hline 3 & 2 & 2 & 2 & 2 & 6640.0615 & -0.0022 \\
\hline 4 & 2 & 3 & 3 & 3 & 7946.5027 & 0.0032 \\
\hline 2 & 2 & 0 & 1 & 1 & 5377.5729 & 0.0046 \\
\hline 3 & 2 & 1 & 2 & 2 & 6664.1848 & -0.0017 \\
\hline 3 & 2 & 2 & 2 & 1 & 6400.4177 & -0.0009 \\
\hline 4 & 2 & 3 & 3 & 2 & 7467.3969 & -0.0002 \\
\hline 5 & 2 & 4 & 5 & 4 & 2380.6612 & 0.0092 \\
\hline 4 & 2 & 3 & 4 & 3 & 2567.9563 & 0.0032 \\
\hline 3 & 2 & 2 & 3 & 2 & 2721.2614 & 0.0115 \\
\hline 2 & 2 & 1 & 2 & 1 & 2837.9711 & 0.0024 \\
\hline 3 & 2 & 1 & 3 & 3 & 3224.4716 & -0.0035 \\
\hline 4 & 2 & 2 & 4 & 4 & 3437.0179 & -0.0088 \\
\hline
\end{tabular}


Table S25. Measured frequencies ( $\left.v_{\mathrm{obs}}\right)$ and residuals $\left(v_{\mathrm{obs}}-v_{\mathrm{cal}}\right)$ of the rotational transitions of the DOH isotopic species of isomer EQA-3 of limonene- $\mathrm{H}_{2} \mathrm{O}$.

\begin{tabular}{cccccccc}
\hline$J^{\prime}$ & $K^{\prime}{ }_{-1}$ & $K^{\prime}+1$ & $J^{\prime \prime}$ & $K^{\prime \prime}{ }_{-1} K^{\prime \prime}+1$ & $v_{\text {obs }} / \mathrm{MHz}$ & $v_{\text {obs }}-v_{\text {calc }} / \mathrm{MHz}$ \\
\hline 2 & 0 & 2 & 1 & 0 & 1 & 2357.2469 & -0.0036 \\
3 & 1 & 3 & 2 & 1 & 2 & 3419.3984 & -0.0011 \\
3 & 0 & 3 & 2 & 0 & 2 & 3523.5329 & -0.0003 \\
3 & 1 & 2 & 2 & 1 & 1 & 3661.0268 & -0.0068 \\
4 & 1 & 4 & 3 & 1 & 3 & 4553.8053 & -0.0018 \\
4 & 0 & 4 & 3 & 0 & 3 & 4675.7120 & -0.0005 \\
4 & 1 & 3 & 3 & 1 & 2 & 4875.0768 & -0.0040 \\
5 & 1 & 5 & 4 & 1 & 4 & 5684.1358 & -0.0016 \\
5 & 0 & 5 & 4 & 0 & 4 & 5811.1403 & 0.0017 \\
5 & 1 & 4 & 4 & 1 & 3 & 6083.1421 & 0.0027 \\
6 & 1 & 6 & 5 & 1 & 5 & 6810.0004 & 0.0039 \\
6 & 0 & 6 & 5 & 0 & 5 & 6929.9101 & 0.0071 \\
6 & 1 & 5 & 5 & 1 & 4 & 7282.9923 & 0.0082 \\
7 & 1 & 7 & 6 & 1 & 6 & 7931.3216 & 0.0068 \\
4 & 2 & 3 & 3 & 2 & 2 & 4720.5248 & -0.0010 \\
4 & 2 & 2 & 3 & 2 & 1 & 4769.2659 & 0.0126 \\
5 & 2 & 4 & 4 & 2 & 3 & 5894.4124 & -0.0009 \\
5 & 2 & 3 & 4 & 2 & 2 & 5988.7548 & -0.0060 \\
6 & 2 & 5 & 5 & 2 & 4 & 7064.1896 & 0.0070 \\
6 & 2 & 4 & 5 & 2 & 3 & 7220.6641 & -0.0036 \\
2 & 1 & 2 & 1 & 0 & 1 & 3217.8343 & 0.0008 \\
4 & 1 & 4 & 3 & 0 & 3 & 5310.2575 & 0.0011 \\
5 & 1 & 5 & 4 & 0 & 4 & 6318.6752 & -0.0060 \\
6 & 1 & 6 & 5 & 0 & 5 & 7317.5345 & -0.0047 \\
5 & 0 & 5 & 4 & 1 & 4 & 5176.5997 & 0.0050 \\
6 & 0 & 6 & 5 & 1 & 5 & 6422.3548 & -0.0057 \\
7 & 0 & 7 & 6 & 1 & 6 & 7647.4802 & -0.0095 \\
5 & 3 & 3 & 4 & 3 & 2 & 5920.6227 & 0.0072 \\
5 & 3 & 2 & 4 & 3 & 1 & 5924.6376 & -0.0009 \\
6 & 3 & 4 & 5 & 3 & 3 & 7108.7778 & -0.0079 \\
6 & 3 & 3 & 5 & 3 & 2 & & -0019 \\
& & & & & &
\end{tabular}

Table S26. Measured frequencies ( $\left.v_{\mathrm{obs}}\right)$ and residuals $\left(v_{\mathrm{obs}}-v_{\text {cal }}\right)$ of the rotational transitions of the HOD isotopic species of isomer EQA-3 of limonene- $\mathrm{H}_{2} \mathrm{O}$.

\begin{tabular}{cccccccc}
\hline$J^{\prime}$ & $K_{-1}^{\prime}$ & $K^{\prime}{ }_{+1} J^{\prime \prime}$ & $K^{\prime \prime}{ }_{-1} K^{\prime \prime}{ }_{+1}$ & $v_{\text {obs }} / \mathrm{MHz}$ & \multicolumn{1}{c}{$v_{\text {obs }}-v_{\text {call }} / \mathrm{MHz}$} \\
\hline 2 & 0 & 2 & 1 & 0 & 1 & 2343.9313 & 0.0012 \\
3 & 1 & 3 & 2 & 1 & 2 & 3394.3510 & -0.0005 \\
3 & 0 & 3 & 2 & 0 & 2 & 3501.9732 & -0.0013 \\
3 & 1 & 2 & 2 & 1 & 1 & 3647.2498 & 0.0000 \\
4 & 1 & 4 & 3 & 1 & 3 & 4519.7479 & -0.0014 \\
4 & 0 & 4 & 3 & 0 & 3 & 4644.2360 & -0.0015 \\
4 & 1 & 3 & 3 & 1 & 2 & 4855.8451 & -0.0001 \\
5 & 1 & 5 & 4 & 1 & 4 & 5640.6184 & -0.0004 \\
5 & 0 & 5 & 4 & 0 & 4 & 5768.0917 & 0.0004 \\
5 & 1 & 4 & 4 & 1 & 3 & 6057.5853 & -0.0021 \\
6 & 1 & 6 & 5 & 1 & 5 & 6756.5757 & -0.0039 \\
6 & 0 & 6 & 5 & 0 & 5 & 6874.2673 & 0.0077 \\
6 & 1 & 5 & 5 & 1 & 4 & 7249.8879 & 0.0058 \\
7 & 1 & 7 & 6 & 1 & 6 & 7867.6269 & -0.0012 \\
7 & 0 & 7 & 6 & 0 & 6 & 7966.8555 & -0.0003 \\
4 & 2 & 3 & 3 & 2 & 2 & 4694.6769 & 0.0025 \\
4 & 2 & 2 & 3 & 2 & 1 & 4749.5273 & -0.0037 \\
5 & 2 & 4 & 4 & 2 & 3 & 5861.2950 & 0.0003 \\
5 & 2 & 3 & 4 & 2 & 2 & 5966.9998 & 0.0038 \\
6 & 2 & 4 & 5 & 2 & 3 & 7197.3161 & -0.0059 \\
5 & 3 & 3 & 4 & 3 & 2 & 5890.7504 & 0.0128 \\
5 & 3 & 2 & 4 & 3 & 1 & 5895.6334 & -0.0061 \\
6 & 3 & 4 & 5 & 3 & 3 & 7073.2439 & 0.0051 \\
6 & 3 & 3 & 5 & 3 & 2 & 7086.1534 & -0.0094 \\
3 & 1 & 3 & 2 & 0 & 2 & 4220.1977 & 0.0004 \\
4 & 1 & 4 & 3 & 0 & 3 & 5237.9750 & 0.0030 \\
5 & 1 & 5 & 4 & 0 & 4 & 6234.3445 & -0.0088 \\
6 & 1 & 6 & 5 & 0 & 5 & 7222.8452 & 0.0035
\end{tabular}


Table S27. Measured frequencies ( $\left.v_{\text {obs }}\right)$ and residuals ( $v_{\text {obs }}-v_{\text {cal }}$ ) of the rotational transitions of the DOD isotopic species of isomer EQA-3 of limonene- $\mathrm{H}_{2} \mathrm{O}$.

\begin{tabular}{|c|c|c|c|c|c|c|c|}
\hline$J^{\prime}$ & $K_{-1}^{\prime}$ & $K_{+1}^{\prime}$ & $J^{\prime \prime}$ & $K^{\prime \prime}{ }_{-1}$ & $K^{\prime \prime}{ }_{+1}$ & $v_{\mathrm{obs}} / \mathrm{MHz}$ & $\nu_{\mathrm{obs}}-\nu_{\text {calc }} / \mathrm{MHz}$ \\
\hline 3 & 1 & 3 & 2 & 1 & 2 & 3374.5294 & 0.0001 \\
\hline 3 & 0 & 3 & 2 & 0 & 2 & 3482.5561 & 0.0023 \\
\hline 3 & 1 & 2 & 2 & 1 & 1 & 3629.0270 & 0.0051 \\
\hline 4 & 1 & 4 & 3 & 1 & 3 & 4493.1921 & -0.0069 \\
\hline 4 & 0 & 4 & 3 & 0 & 3 & 4617.8511 & 0.0039 \\
\hline 4 & 1 & 3 & 3 & 1 & 2 & 4831.3871 & 0.0061 \\
\hline 5 & 1 & 5 & 4 & 1 & 4 & 5607.2607 & 0.0009 \\
\hline 5 & 0 & 5 & 4 & 0 & 4 & 5734.4491 & -0.0007 \\
\hline 5 & 1 & 4 & 4 & 1 & 3 & 6026.7296 & 0.0063 \\
\hline 6 & 1 & 6 & 5 & 1 & 5 & 6716.3359 & -0.0007 \\
\hline 6 & 0 & 6 & 5 & 0 & 5 & 6833.2283 & 0.0032 \\
\hline 6 & 1 & 5 & 5 & 1 & 4 & 7212.3921 & 0.0086 \\
\hline 7 & 1 & 7 & 6 & 1 & 6 & 7820.4375 & -0.0027 \\
\hline 7 & 0 & 7 & 6 & 0 & 6 & 7918.4840 & 0.0022 \\
\hline 3 & 2 & 2 & 2 & 2 & 1 & 3505.3586 & 0.0009 \\
\hline 3 & 2 & 1 & 2 & 2 & 0 & 3528.1877 & -0.0009 \\
\hline 4 & 2 & 3 & 3 & 2 & 2 & 4669.3217 & 0.0021 \\
\hline 4 & 2 & 2 & 3 & 2 & 1 & 4725.2960 & -0.0017 \\
\hline 5 & 2 & 4 & 4 & 2 & 3 & 5829.4571 & 0.0049 \\
\hline 5 & 2 & 3 & 4 & 2 & 2 & 5937.2036 & -0.0025 \\
\hline 6 & 2 & 5 & 5 & 2 & 4 & 6984.8526 & 0.0048 \\
\hline 6 & 2 & 4 & 5 & 2 & 3 & 7162.0089 & -0.0031 \\
\hline 4 & 3 & 2 & 3 & 3 & 1 & 4684.5535 & -0.0038 \\
\hline 4 & 3 & 1 & 3 & 3 & 0 & 4686.0212 & 0.0036 \\
\hline 5 & 3 & 3 & 4 & 3 & 2 & 5859.5053 & 0.0075 \\
\hline 5 & 3 & 2 & 4 & 3 & 1 & 5864.5663 & -0.0104 \\
\hline 6 & 3 & 4 & 5 & 3 & 3 & 7035.7967 & -0.0010 \\
\hline 6 & 3 & 3 & 5 & 3 & 2 & 7049.1788 & -0.0049 \\
\hline 3 & 1 & 3 & 2 & 0 & 2 & 4191.7367 & -0.0191 \\
\hline 4 & 1 & 4 & 3 & 0 & 3 & 5202.3940 & -0.0070 \\
\hline 5 & 1 & 5 & 4 & 0 & 4 & 6191.8181 & 0.0045 \\
\hline 6 & 1 & 6 & 5 & 0 & 5 & 7173.7104 & 0.0099 \\
\hline 6 & 0 & 6 & 5 & 1 & 5 & 6375.8526 & -0.0087 \\
\hline 7 & 0 & 7 & 6 & 1 & 6 & 7577.9998 & -0.0068 \\
\hline
\end{tabular}


Table S28. Measured frequencies ( $v_{\text {obs }}$ ) and residuals ( $v_{\text {obs }}-v_{\text {cal }}$ ) of the rotational transitions of the $\mathrm{H}_{2}{ }^{18} \mathrm{O}$ isotopic species of isomer EQA-3 of limonene- $\mathrm{H}_{2} \mathrm{O}$.

\begin{tabular}{|c|c|c|c|c|c|c|}
\hline$J^{\prime}$ & $K_{-1}^{\prime}$ & $K^{\prime}+1$ & $J^{\prime \prime}$ & $K^{\prime \prime}{ }_{-1} K^{\prime \prime}{ }_{+1}$ & $v_{\mathrm{obs}} / \mathrm{MHz}$ & $v_{\text {obs }}-v_{\text {calc }} / \mathrm{MHz}$ \\
\hline 2 & 1 & 2 & 1 & 1 & 2254.7057 & -0.0003 \\
\hline 2 & 0 & 2 & 1 & 0 & 2335.1995 & -0.0025 \\
\hline 2 & 1 & 1 & 1 & 10 & 2427.7128 & -0.0002 \\
\hline 3 & 1 & 3 & 2 & 2 & 3378.4172 & -0.0004 \\
\hline 3 & 0 & 3 & 2 & 0 & 3487.9537 & -0.0007 \\
\hline 3 & 1 & 2 & 2 & 1 & 3637.6606 & -0.0013 \\
\hline 4 & 1 & 4 & 3 & 13 & 4498.1147 & -0.0010 \\
\hline 4 & 0 & 4 & 3 & 3 & 4623.9527 & 0.0006 \\
\hline 4 & 1 & 3 & 3 & 1 & 4842.5495 & -0.0001 \\
\hline 5 & 1 & 5 & 4 & 4 & 5613.0252 & 0.0009 \\
\hline 5 & 0 & 5 & 4 & 0 & 5740.6162 & 0.0017 \\
\hline 5 & 1 & 4 & 4 & 1 & 6040.0668 & 0.0018 \\
\hline 6 & 1 & 6 & 5 & 5 & 6722.7749 & 0.0005 \\
\hline 6 & 0 & 6 & 5 & 0 & 6839.0775 & 0.0015 \\
\hline 6 & 1 & 5 & 5 & 1 & 7227.3951 & 0.0042 \\
\hline 7 & 1 & 7 & 6 & 1 & 7827.4055 & 0.0006 \\
\hline 7 & 0 & 7 & 6 & 0 & 7924.0347 & 0.0018 \\
\hline 3 & 2 & 2 & 2 & 2 & 3511.7843 & 0.0020 \\
\hline 3 & 2 & 1 & 2 & 2 & 3535.6376 & -0.0048 \\
\hline 4 & 2 & 3 & 3 & 2 & 4677.6800 & 0.0003 \\
\hline 4 & 2 & 2 & 3 & 1 & 4736.1182 & -0.0046 \\
\hline 5 & 2 & 4 & 4 & 2 & 5839.5745 & 0.0012 \\
\hline 5 & 2 & 3 & 4 & 2 & 5951.8593 & -0.0066 \\
\hline 6 & 2 & 5 & 5 & 2 & 6996.5206 & 0.0040 \\
\hline 6 & 2 & 4 & 5 & 2 & 7180.6429 & -0.0041 \\
\hline 4 & 3 & 2 & 3 & 3 & 4693.5926 & 0.0065 \\
\hline 5 & 3 & 3 & 4 & 3 & 5870.9178 & 0.0042 \\
\hline 6 & 3 & 4 & 5 & 3 & 7049.6086 & 0.0017 \\
\hline 6 & 3 & 3 & 5 & 3 & 7063.9487 & -0.0066 \\
\hline 3 & 1 & 2 & 2 & 0 & 4705.2927 & 0.0003 \\
\hline 4 & 1 & 3 & 3 & 0 & 6059.8861 & -0.0016 \\
\hline 5 & 1 & 4 & 4 & 0 & 7476.0081 & 0.0076 \\
\hline 2 & 2 & 0 & 1 & 1 & 5099.5635 & 0.0071 \\
\hline 2 & 2 & 1 & 1 & 1 & 5180.0494 & 0.0015 \\
\hline 3 & 2 & 1 & 2 & 1 & 6207.4900 & 0.0042 \\
\hline 3 & 2 & 2 & 2 & 1 & 6437.1269 & 0.0027 \\
\hline 4 & 2 & 2 & 3 & 2 & 7305.9416 & -0.0053 \\
\hline 4 & 2 & 3 & 3 & 1 & 7736.3842 & -0.0021 \\
\hline 3 & 0 & 3 & 2 & 1 & 2679.8352 & 0.0002 \\
\hline 4 & 0 & 4 & 3 & 1 & 3925.3738 & 0.0041 \\
\hline 5 & 0 & 5 & 4 & 1 & 5167.8600 & -0.0084 \\
\hline 6 & 0 & 6 & 5 & 1 & 6393.9276 & 0.0075 \\
\hline 7 & 0 & 7 & 6 & 1 & 7595.1706 & -0.0081 \\
\hline 2 & 1 & 2 & 1 & 0 & 3143.3206 & -0.0007 \\
\hline 3 & 1 & 3 & 2 & 0 & 4186.5340 & -0.0028 \\
\hline 4 & 1 & 4 & 3 & 0 & 5196.6995 & 0.0013 \\
\hline 5 & 1 & 5 & 4 & 0 & 6185.7721 & 0.0017 \\
\hline 6 & 1 & 6 & 5 & 0 & 7167.9296 & -0.0006 \\
\hline 2 & 2 & 1 & 1 & 1 & 5093.5299 & -0.0136 \\
\hline 2 & 2 & 0 & 1 & 1 & 5186.0698 & 0.0091 \\
\hline 3 & 2 & 1 & 2 & 1 & 6466.9901 & -0.0070 \\
\hline
\end{tabular}


Table S29. Measured frequencies ( $\left.v_{\text {obs }}\right)$ and residuals ( $v_{\text {obs }}-v_{\text {cal }}$ ) of the rotational transitions of the parent species of isomer EQA-4 of limonene- $\mathrm{H}_{2} \mathrm{O}$.

\begin{tabular}{|c|c|c|c|c|c|c|}
\hline$J^{\prime}$ & $K_{-1}^{\prime}$ & $K^{\prime}+1$ & $J^{\prime \prime}$ & $K^{\prime \prime}{ }_{-1}^{\prime \prime} K^{\prime \prime}$ & $v_{\mathrm{obs}} / \mathrm{MHz}$ & $v_{\text {obs }}-v_{\text {calc }} / \mathrm{MHz}$ \\
\hline 2 & 0 & 2 & 1 & 0 & 2361.4367 & -0.0007 \\
\hline 2 & 1 & 1 & 1 & 0 & 2452.8814 & 0.0041 \\
\hline 3 & 0 & 3 & 2 & 2 & 3528.2274 & 0.0023 \\
\hline 3 & 1 & 3 & 2 & 2 & 3418.4691 & 0.0002 \\
\hline 3 & 1 & 2 & 2 & 1 & 3675.6670 & 0.0014 \\
\hline 4 & 0 & 4 & 3 & 3 & 4679.1924 & 0.0014 \\
\hline 4 & 1 & 4 & 3 & 3 & 4551.8962 & -0.0002 \\
\hline 4 & 1 & 3 & 3 & 2 & 4893.7571 & 0.0131 \\
\hline 5 & 0 & 5 & 4 & 4 & 5811.6254 & 0.0011 \\
\hline 5 & 1 & 5 & 4 & 4 & 5680.7767 & -0.0012 \\
\hline 5 & 1 & 4 & 4 & 3 & 6104.9900 & 0.0004 \\
\hline 6 & 0 & 6 & 5 & 5 & 6926.1142 & 0.0002 \\
\hline 6 & 1 & 6 & 5 & 5 & 6804.7210 & 0.0022 \\
\hline 7 & 1 & 7 & 6 & 6 & 7923.6976 & -0.0019 \\
\hline 3 & 2 & 2 & 2 & 1 & 3550.5781 & 0.0028 \\
\hline 3 & 2 & 1 & 2 & 0 & 3572.9484 & -0.0047 \\
\hline 4 & 2 & 3 & 3 & 2 & 4729.7066 & 0.0021 \\
\hline 4 & 2 & 2 & 3 & 1 & 4784.6327 & -0.0014 \\
\hline 5 & 2 & 4 & 4 & 3 & 5905.0877 & 0.0037 \\
\hline 5 & 2 & 3 & 4 & 2 & 6011.0428 & -0.0002 \\
\hline 6 & 2 & 5 & 5 & 4 & 7075.8286 & 0.0071 \\
\hline 6 & 2 & 4 & 5 & 3 & 7250.5689 & -0.0101 \\
\hline 3 & 1 & 3 & 2 & 2 & 4274.1195 & -0.0006 \\
\hline 3 & 0 & 3 & 2 & 2 & 2672.5735 & -0.0004 \\
\hline 4 & 1 & 4 & 3 & 3 & 5297.7917 & 0.0003 \\
\hline 4 & 0 & 4 & 3 & 3 & 3933.2980 & 0.0021 \\
\hline 5 & 1 & 5 & 4 & 4 & 6299.3765 & -0.0018 \\
\hline 5 & 0 & 5 & 4 & 4 & 5193.0244 & 0.0006 \\
\hline 6 & 1 & 6 & 5 & 5 & 7292.4722 & -0.0006 \\
\hline 6 & 0 & 6 & 5 & 5 & 6438.3579 & -0.0021 \\
\hline 7 & 0 & 7 & 6 & 6 & 7660.2924 & -0.0037 \\
\hline 2 & 2 & 0 & 1 & 1 & 5351.8116 & 0.0085 \\
\hline 2 & 2 & 1 & 1 & 1 & 5260.3489 & -0.0094 \\
\hline 3 & 2 & 2 & 2 & 1 & 6358.0539 & -0.0023 \\
\hline 3 & 2 & 1 & 2 & 1 & 6643.5005 & 0.0014 \\
\hline 4 & 2 & 3 & 3 & 1 & 7412.0968 & 0.0017 \\
\hline 2 & 1 & 1 & 1 & 0 & 3474.5227 & 0.0035 \\
\hline 3 & 1 & 2 & 2 & 0 & 4788.7470 & -0.0004 \\
\hline 4 & 1 & 3 & 3 & 0 & 6154.2688 & 0.0025 \\
\hline 5 & 1 & 4 & 4 & 0 & 7580.0652 & 0.0003 \\
\hline 3 & 2 & 2 & 2 & 1 & 6615.4861 & -0.0007 \\
\hline 4 & 2 & 3 & 3 & 1 & 7926.7219 & -0.0006 \\
\hline 4 & 2 & 2 & 3 & 1 & 7495.0311 & -0.0059 \\
\hline 2 & 2 & 1 & 1 & 1 & 5346.1637 & -0.0049 \\
\hline 2 & 2 & 0 & 1 & 1 & 5265.9933 & 0.0006 \\
\hline 3 & 2 & 1 & 2 & 1 & 6386.0686 & 0.0001 \\
\hline 5 & 1 & 4 & 4 & 2 & 3586.6346 & -0.0038 \\
\hline 6 & 1 & 5 & 5 & 2 & 4799.4858 & 0.0074 \\
\hline 7 & 1 & 6 & 6 & 2 & 6045.1090 & 0.0069 \\
\hline 3 & 0 & 3 & 2 & 1 & 2415.1374 & -0.0059 \\
\hline 4 & 0 & 4 & 3 & 1 & 3418.6631 & -0.0057 \\
\hline 5 & 1 & 4 & 4 & 2 & 3503.6936 & -0.0030 \\
\hline 2 & 2 & 0 & 2 & 1 & 3070.5461 & 0.0001 \\
\hline 3 & 2 & 1 & 3 & 1 & 2710.4038 & 0.0009 \\
\hline 4 & 2 & 2 & 4 & 1 & 2601.2900 & -0.0031 \\
\hline 5 & 2 & 3 & 5 & 1 & 2507.3434 & -0.0031 \\
\hline 3 & 2 & 2 & 3 & 1 & 3197.0173 & -0.0007 \\
\hline 4 & 2 & 3 & 4 & 1 & 3374.8278 & 0.0017 \\
\hline 5 & 2 & 4 & 5 & 1 & 2318.4550 & 0.0094 \\
\hline 7 & 1 & 6 & 6 & 2 & 6408.7581 & -0.0025 \\
\hline 5 & 1 & 4 & 4 & 2 & 3586.6349 & -0.0035 \\
\hline
\end{tabular}


Table S30. Measured frequencies ( $\left.v_{\text {obs }}\right)$ and residuals ( $v_{\text {obs }}-v_{\text {cal }}$ ) of the rotational transitions of the DOH isotopic species of isomer EQA-4 of limonene- $\mathrm{H}_{2} \mathrm{O}$.

\begin{tabular}{|c|c|c|c|c|c|c|}
\hline$J^{\prime}$ & $K_{-1}^{\prime}$ & $K^{\prime}+1$ & $J^{\prime \prime}$ & $K^{\prime \prime}{ }_{-1} K^{\prime \prime}{ }_{+1}$ & $v_{\mathrm{obs}} / \mathrm{MHz}$ & $v_{\text {obs }}-v_{\text {calc }} / \mathrm{MHz}$ \\
\hline 4 & 1 & 4 & 3 & 1 & 4525.0208 & 0.0021 \\
\hline 4 & 0 & 4 & 3 & 0 & 4652.7234 & 0.0010 \\
\hline 4 & 1 & 3 & 3 & 12 & 4870.1620 & -0.0067 \\
\hline 3 & 1 & 3 & 2 & 1 & 3398.4400 & 0.0020 \\
\hline 3 & 0 & 3 & 2 & 0 & 3508.9104 & 0.0103 \\
\hline 3 & 1 & 2 & 2 & 1 & 3658.1504 & -0.0054 \\
\hline 5 & 1 & 5 & 4 & 1 & 5646.9402 & 0.0006 \\
\hline 5 & 0 & 5 & 4 & 0 & 5777.6058 & 0.0015 \\
\hline 5 & 1 & 4 & 4 & 1 & 6075.1163 & -0.0016 \\
\hline 6 & 1 & 6 & 5 & 1 & 6763.8105 & -0.0019 \\
\hline 6 & 0 & 6 & 5 & 0 & 6884.3213 & 0.0012 \\
\hline 6 & 1 & 5 & 5 & 1 & 7270.3394 & 0.0120 \\
\hline 3 & 2 & 2 & 2 & 2 & 3531.9143 & 0.0028 \\
\hline 3 & 2 & 1 & 2 & 2 & 3554.9379 & -0.0111 \\
\hline 4 & 2 & 3 & 3 & 2 & 4704.6981 & 0.0119 \\
\hline 4 & 2 & 2 & 3 & 2 & 4761.1950 & 0.0007 \\
\hline 5 & 2 & 4 & 4 & 2 & 5873.5988 & 0.0001 \\
\hline 5 & 2 & 3 & 4 & 2 & 5982.4481 & -0.0090 \\
\hline 6 & 2 & 5 & 5 & 2 & 7037.7417 & 0.0097 \\
\hline 6 & 2 & 4 & 5 & 2 & 7216.9201 & 0.0017 \\
\hline 4 & 0 & 4 & 3 & 1 & 3918.1921 & 0.0111 \\
\hline 5 & 0 & 5 & 4 & 1 & 5170.7633 & -0.0033 \\
\hline 6 & 0 & 6 & 5 & 1 & 6408.1385 & -0.0087 \\
\hline 7 & 0 & 7 & 6 & 1 & 7621.4586 & -0.0089 \\
\hline 2 & 1 & 2 & 1 & 0 & 3193.8254 & -0.0049 \\
\hline 3 & 1 & 3 & 2 & 0 & 4243.4396 & -0.0018 \\
\hline 4 & 1 & 4 & 3 & 0 & 5259.5474 & -0.0126 \\
\hline 5 & 1 & 5 & 4 & 0 & 6253.7700 & -0.0073 \\
\hline 6 & 1 & 6 & 5 & 0 & 7239.9998 & 0.0144 \\
\hline 2 & 1 & 1 & 1 & 0 & 3453.8025 & 0.0056 \\
\hline 2 & 2 & 1 & 1 & 1 & 5305.5066 & 0.0004 \\
\hline 4 & 2 & 2 & 3 & 1 & 7441.3455 & -0.0142 \\
\hline 4 & 2 & 3 & 3 & 1 & 7875.6855 & -0.0109 \\
\hline 2 & 2 & 0 & 1 & 1 & 5311.3197 & 0.0112 \\
\hline 3 & 2 & 1 & 2 & 1 & 6598.2976 & 0.0096 \\
\hline 3 & 2 & 2 & 2 & 1 & 6569.4523 & 0.0042 \\
\hline
\end{tabular}


Table S31. Measured frequencies ( $\left.v_{\text {obs }}\right)$ and residuals ( $v_{\text {obs }}-v_{\text {cal }}$ ) of the rotational transitions of the HOD isotopic species of isomer EQA-4 of limonene- $\mathrm{H}_{2} \mathrm{O}$.

\begin{tabular}{|c|c|c|c|c|c|c|}
\hline$J^{\prime}$ & $K_{-1}^{\prime}$ & $K^{\prime}+1$ & $J^{\prime \prime}$ & $K^{\prime \prime}{ }_{-1} K^{\prime \prime}+1$ & $v_{\mathrm{obs}} / \mathrm{MHz}$ & $v_{\text {obs }}-v_{\text {calc }} / \mathrm{MHz}$ \\
\hline 3 & 1 & 3 & 2 & 2 & 3374.2135 & 0.0037 \\
\hline 3 & 0 & 3 & 2 & 0 & 3487.7661 & 0.0019 \\
\hline 4 & 1 & 4 & 3 & 13 & 4492.0140 & -0.0016 \\
\hline 4 & 0 & 4 & 3 & 3 & 4621.6673 & -0.0074 \\
\hline 4 & 1 & 3 & 3 & 12 & 4851.2754 & -0.0005 \\
\hline 5 & 1 & 5 & 4 & 1 & 5604.6982 & 0.0031 \\
\hline 5 & 0 & 5 & 4 & 0 & 5735.0185 & 0.0019 \\
\hline 5 & 1 & 4 & 4 & 3 & 6049.8691 & 0.0033 \\
\hline 6 & 1 & 6 & 5 & 1 & 6711.8943 & 0.0085 \\
\hline 6 & 0 & 6 & 5 & 0 & 6829.3483 & -0.0026 \\
\hline 6 & 1 & 5 & 5 & 1 & 7237.3675 & 0.0046 \\
\hline 7 & 1 & 7 & 6 & 1 & 7813.6601 & -0.0097 \\
\hline 2 & 0 & 2 & 1 & 0 & 2335.8503 & -0.0052 \\
\hline 3 & 2 & 1 & 2 & 2 & 3539.2428 & -0.0076 \\
\hline 4 & 2 & 3 & 3 & 2 & 4679.6032 & 0.0094 \\
\hline 4 & 2 & 2 & 3 & 2 & 4742.5903 & -0.0013 \\
\hline 5 & 2 & 4 & 4 & 2 & 5841.3760 & 0.0012 \\
\hline 5 & 2 & 3 & 4 & 2 & 5962.1108 & 0.0004 \\
\hline 6 & 2 & 5 & 5 & 2 & 6997.8161 & -0.0053 \\
\hline 6 & 2 & 4 & 5 & 3 & 7195.0615 & -0.0030 \\
\hline 3 & 1 & 3 & 2 & 2 & 4184.4836 & 0.0004 \\
\hline 4 & 1 & 4 & 3 & 3 & 5188.7365 & 0.0019 \\
\hline 5 & 1 & 5 & 4 & 0 & 6171.7587 & 0.0038 \\
\hline 6 & 1 & 6 & 5 & 5 & 7148.6288 & 0.0047 \\
\hline 4 & 0 & 4 & 3 & 1 & 3924.9352 & -0.0205 \\
\hline 5 & 0 & 5 & 4 & 1 & 5167.9633 & 0.0065 \\
\hline 6 & 0 & 6 & 5 & 1 & 6392.6139 & 0.0013 \\
\hline 7 & 0 & 7 & 6 & 1 & 7590.7373 & 0.0012 \\
\hline 2 & 1 & 1 & 1 & 0 & 3416.8991 & 0.0053 \\
\hline 3 & 1 & 2 & 2 & 2 & 4725.7280 & 0.0106 \\
\hline 4 & 1 & 3 & 3 & 0 & 6089.2292 & 0.0002 \\
\hline 5 & 1 & 4 & 4 & 4 & 7517.4183 & -0.0018 \\
\hline 2 & 2 & 0 & 1 & 1 & 5121.1990 & 0.0019 \\
\hline 3 & 2 & 1 & 2 & 1 & 6227.8457 & -0.0016 \\
\hline 3 & 2 & 2 & 2 & 1 & 6466.3576 & -0.0054 \\
\hline 4 & 2 & 2 & 3 & 1 & 7325.7584 & -0.0012 \\
\hline 2 & 2 & 1 & 1 & 1 & 5114.6977 & -0.0054 \\
\hline 2 & 2 & 0 & 1 & 1 & 5211.4533 & 0.0009 \\
\hline 3 & 2 & 2 & 2 & 1 & 6195.5914 & -0.0067 \\
\hline 3 & 2 & 1 & 2 & 1 & 6498.6122 & 0.0000 \\
\hline 4 & 2 & 3 & 3 & 1 & 7230.5115 & -0.0013 \\
\hline 4 & 2 & 2 & 3 & 1 & 7867.0006 & 0.0067 \\
\hline
\end{tabular}


Table S32. Measured frequencies ( $\left.v_{\text {obs }}\right)$ and residuals ( $v_{\text {obs }}-v_{\text {cal }}$ ) of the rotational transitions of the DOD isotopic species of isomer EQA-4 of limonene- $\mathrm{H}_{2} \mathrm{O}$.

\begin{tabular}{|c|c|c|c|c|c|c|}
\hline$J^{\prime}$ & $K_{-1}^{\prime}$ & $K^{\prime}+1$ & $J^{\prime \prime}$ & $K^{\prime \prime}{ }_{-1} K^{\prime \prime}{ }_{+1}$ & $v_{\mathrm{obs}} / \mathrm{MHz}$ & $v_{\text {obs }}-v_{\text {calc }} / \mathrm{MHz}$ \\
\hline 3 & 1 & 3 & 2 & 12 & 3354.7642 & 0.0014 \\
\hline 3 & 0 & 3 & 2 & 0 & 3468.7985 & -0.0027 \\
\hline 3 & 1 & 2 & 2 & 11 & 3627.2056 & -0.0051 \\
\hline 4 & 1 & 4 & 3 & 1 & 4465.9324 & -0.0003 \\
\hline 4 & 0 & 4 & 3 & 0 & 4595.7622 & -0.0017 \\
\hline 4 & 1 & 3 & 3 & 1 & 4827.7696 & -0.0037 \\
\hline 5 & 1 & 5 & 4 & 4 & 5571.8766 & -0.0001 \\
\hline 5 & 0 & 5 & 4 & 0 & 5701.8274 & 0.0023 \\
\hline 5 & 1 & 4 & 4 & 1 & 6020.1114 & -0.0011 \\
\hline 6 & 1 & 6 & 5 & 1 & 6672.2378 & -0.0029 \\
\hline 6 & 0 & 6 & 5 & 0 & 6788.7453 & 0.0010 \\
\hline 6 & 1 & 5 & 5 & 1 & 7201.0533 & 0.0048 \\
\hline 7 & 1 & 7 & 6 & 1 & 7767.1275 & -0.0008 \\
\hline 7 & 0 & 7 & 6 & 0 & 7862.0954 & 0.0002 \\
\hline 4 & 2 & 3 & 3 & 2 & 4654.9941 & 0.0017 \\
\hline 4 & 2 & 2 & 3 & 2 & 4719.4055 & -0.0105 \\
\hline 5 & 2 & 4 & 4 & 2 & 5810.4301 & 0.0008 \\
\hline 5 & 2 & 3 & 4 & 2 & 5933.7367 & -0.0059 \\
\hline 6 & 2 & 5 & 5 & 4 & 6960.4142 & 0.0068 \\
\hline 6 & 2 & 4 & 5 & 2 & 7161.5008 & 0.0012 \\
\hline 4 & 0 & 4 & 3 & 1 & 3908.5691 & 0.0138 \\
\hline 5 & 0 & 5 & 4 & 1 & 5144.4448 & -0.0030 \\
\hline 6 & 0 & 6 & 5 & 1 & 6361.3093 & -0.0062 \\
\hline 7 & 0 & 7 & 6 & 6 & 7551.1650 & -0.0048 \\
\hline 5 & 1 & 5 & 4 & 4 & 6129.2549 & 0.0010 \\
\hline 6 & 1 & 6 & 5 & 0 & 7099.6773 & 0.0077 \\
\hline 2 & 1 & 1 & 1 & 1 & 3397.4742 & 0.0082 \\
\hline 3 & 1 & 2 & 2 & 2 & 4701.2167 & 0.0016 \\
\hline 4 & 1 & 3 & 3 & 3 & 6060.1897 & 0.0024 \\
\hline 5 & 1 & 4 & 4 & 0 & 7484.5386 & 0.0028 \\
\hline 2 & 2 & 0 & 1 & 1 & 5175.1490 & 0.0017 \\
\hline 3 & 2 & 1 & 2 & 2 & 6457.4553 & 0.0027 \\
\hline 4 & 2 & 2 & 3 & 1 & 7822.1087 & 0.0029 \\
\hline 4 & 2 & 2 & 3 & 1 & 7276.8913 & -0.0091 \\
\hline 3 & 2 & 2 & 2 & 1 & 6424.4444 & -0.0043 \\
\hline 4 & 2 & 3 & 3 & 1 & 7724.6775 & -0.0008 \\
\hline
\end{tabular}


Table S33. Measured frequencies ( $\left.v_{\mathrm{obs}}\right)$ and residuals ( $\left.v_{\mathrm{obs}}-v_{\mathrm{cal}}\right)$ of the rotational transitions of the $\mathrm{H}_{2}{ }^{18} \mathrm{O}$ isotopic species of isomer EQA-4 of limonene- $\mathrm{H}_{2} \mathrm{O}$.

\begin{tabular}{|c|c|c|c|c|c|c|}
\hline$J^{\prime}$ & $K_{-1}^{\prime}$ & $K_{+1}^{\prime}$ & $J^{\prime \prime}$ & $K^{\prime \prime}{ }_{-1} K^{\prime \prime}{ }_{+1}$ & $v_{\mathrm{obs}} / \mathrm{MHz}$ & $v_{\text {obs }}-v_{\text {calc }} / \mathrm{MHz}$ \\
\hline 2 & 0 & 2 & 1 & $\begin{array}{ll}0 & 1\end{array}$ & 2325.5302 & -0.0051 \\
\hline 2 & 1 & 1 & 1 & 10 & 2424.2878 & -0.0054 \\
\hline 3 & 1 & 3 & 2 & 12 & 3356.5320 & 0.0010 \\
\hline 3 & 0 & 3 & 2 & 02 & 3471.4583 & 0.0012 \\
\hline 3 & 1 & 2 & 2 & 11 & 3631.9932 & 0.0038 \\
\hline 4 & 1 & 4 & 3 & 13 & 4468.1004 & -0.0007 \\
\hline 4 & 0 & 4 & 3 & 03 & 4598.5323 & -0.0024 \\
\hline 4 & 1 & 3 & 3 & 12 & 4833.8970 & 0.0046 \\
\hline 5 & 1 & 5 & 4 & 14 & 5574.3288 & 0.0086 \\
\hline 5 & 0 & 5 & 4 & 04 & 5704.2931 & 0.0047 \\
\hline 5 & 1 & 4 & 4 & 13 & 6027.3160 & 0.0031 \\
\hline 6 & 1 & 6 & 5 & 15 & 6674.8336 & -0.0089 \\
\hline 6 & 0 & 6 & 5 & $0 \quad 5$ & 6790.6944 & -0.0060 \\
\hline 6 & 1 & 5 & 5 & 14 & 7208.9653 & 0.0072 \\
\hline 7 & 1 & 7 & 6 & 16 & 7769.8092 & 0.0149 \\
\hline 7 & 0 & 7 & 6 & 06 & 7863.6178 & -0.0030 \\
\hline 3 & 2 & 2 & 2 & 21 & 3498.5328 & 0.0078 \\
\hline 4 & 2 & 3 & 3 & 22 & 4659.3679 & 0.0025 \\
\hline 4 & 2 & 2 & 3 & 21 & 4725.5381 & -0.0023 \\
\hline 5 & 2 & 3 & 4 & 22 & 5942.1560 & -0.0037 \\
\hline 6 & 2 & 4 & 5 & 23 & 7172.2341 & -0.0089 \\
\hline 2 & 1 & 2 & 1 & 01 & 3120.0220 & -0.0002 \\
\hline 3 & 1 & 3 & 2 & 02 & 4151.0209 & 0.0029 \\
\hline 4 & 1 & 4 & 3 & 03 & 5147.6595 & -0.0024 \\
\hline 5 & 1 & 5 & 4 & 04 & 6123.4463 & -0.0012 \\
\hline 6 & 1 & 6 & 5 & $0 \quad 5$ & 7093.9969 & -0.0047 \\
\hline 3 & 0 & 3 & 2 & 12 & 2676.9676 & -0.0025 \\
\hline 4 & 0 & 4 & 3 & 13 & 3918.9706 & -0.0031 \\
\hline 5 & 0 & 5 & 4 & 14 & 5155.1636 & 0.0025 \\
\hline 6 & 0 & 6 & 5 & 15 & 6371.5392 & -0.0021 \\
\hline 7 & 0 & 7 & 6 & 16 & 7560.3161 & -0.0036 \\
\hline 2 & 2 & 1 & 1 & 10 & 5063.0359 & 0.0061 \\
\hline 2 & 2 & 0 & 1 & 11 & 5161.7810 & -0.0116 \\
\hline 3 & 2 & 2 & 2 & 11 & 6137.2602 & -0.0014 \\
\hline 3 & 2 & 1 & 2 & 12 & 6446.9718 & 0.0011 \\
\hline 4 & 2 & 3 & 3 & 12 & 7164.6395 & 0.0020 \\
\hline 2 & 1 & 1 & 1 & 01 & 3395.8044 & 0.0009 \\
\hline 3 & 1 & 2 & 2 & 02 & 4702.2533 & -0.0044 \\
\hline 4 & 1 & 3 & 3 & 03 & 6064.6970 & 0.0040 \\
\hline 5 & 1 & 4 & 4 & 04 & 7493.4722 & 0.0010 \\
\hline 6 & 1 & 5 & 5 & 24 & 5089.8620 & -0.0020 \\
\hline 7 & 1 & 6 & 6 & 25 & 6498.4903 & 0.0015 \\
\hline 2 & 2 & 0 & 1 & 10 & 5069.8687 & 0.0037 \\
\hline 2 & 2 & 1 & 1 & 11 & 5154.9546 & -0.0027 \\
\hline 3 & 2 & 1 & 2 & 11 & 6171.1904 & 0.0010 \\
\hline 3 & 2 & 2 & 2 & 12 & 6413.0429 & 0.0002 \\
\hline 4 & 2 & 2 & 3 & 12 & 7264.7398 & -0.0005 \\
\hline 4 & 2 & 3 & 3 & 13 & 7715.8770 & -0.0002 \\
\hline
\end{tabular}


Table S34. Measured frequencies ( $\left.v_{\text {obs }}\right)$ and residuals ( $v_{\text {obs }}-v_{\text {cal }}$ ) of the rotational transitions of the parent species of isomer EQC-4 of limonene- $\mathrm{H}_{2} \mathrm{O}$.

\begin{tabular}{|c|c|c|c|c|c|c|}
\hline$J^{\prime}$ & $K_{-1}^{\prime}$ & $K^{\prime}+1$ & $J^{\prime \prime}$ & $K^{\prime \prime}{ }_{-1} K^{\prime \prime}+1$ & $v_{\mathrm{obs}} / \mathrm{MHz}$ & $v_{\text {obs }}-v_{\text {calc }} / \mathrm{MHz}$ \\
\hline 2 & 0 & 2 & 1 & 0 & 2382.0295 & -0.0020 \\
\hline 2 & 1 & 2 & 1 & 1 & 2294.3756 & 0.0016 \\
\hline 2 & 1 & 1 & 1 & 10 & 2483.7891 & -0.0020 \\
\hline 3 & 1 & 3 & 2 & 2 & 3437.3076 & 0.0070 \\
\hline 3 & 0 & 3 & 2 & 2 & 3555.6598 & 0.0001 \\
\hline 3 & 1 & 2 & 2 & 1 & 3721.0927 & 0.0007 \\
\hline 4 & 0 & 4 & 3 & 3 & 4709.8416 & -0.0016 \\
\hline 4 & 1 & 4 & 3 & 13 & 4575.5614 & -0.0005 \\
\hline 4 & 1 & 3 & 3 & 2 & 4952.4129 & 0.0019 \\
\hline 5 & 0 & 5 & 4 & 4 & 5842.0365 & -0.0004 \\
\hline 5 & 1 & 5 & 4 & 4 & 5708.3012 & -0.0025 \\
\hline 5 & 1 & 4 & 4 & 1 & 6174.9761 & 0.0056 \\
\hline 6 & 0 & 6 & 5 & 0 & 6954.3053 & -0.0024 \\
\hline 6 & 1 & 6 & 5 & 1 & 6835.1675 & -0.0040 \\
\hline 6 & 1 & 5 & 5 & 1 & 7385.3814 & 0.0123 \\
\hline 7 & 1 & 7 & 6 & 1 & 7956.2959 & -0.0027 \\
\hline 3 & 2 & 1 & 2 & 2 & 3611.5535 & -0.0089 \\
\hline 3 & 2 & 2 & 2 & 2 & 3583.6040 & 0.0062 \\
\hline 4 & 2 & 3 & 3 & 2 & 4772.6263 & 0.0023 \\
\hline 4 & 2 & 2 & 3 & 2 & 4840.9171 & -0.0052 \\
\hline 5 & 2 & 4 & 4 & 2 & 5956.9689 & 0.0090 \\
\hline 5 & 2 & 3 & 4 & 2 & 6087.4895 & -0.0059 \\
\hline 6 & 2 & 5 & 5 & 2 & 7135.5150 & 0.0089 \\
\hline 6 & 2 & 4 & 5 & 2 & 7347.9213 & -0.0026 \\
\hline 4 & 3 & 2 & 3 & 1 & 4791.2482 & -0.0004 \\
\hline 5 & 3 & 3 & 4 & 3 & 5993.5415 & -0.0075 \\
\hline 5 & 3 & 2 & 4 & 3 & 6000.3810 & -0.0112 \\
\hline 6 & 3 & 4 & 5 & 3 & 7197.2931 & 0.0098 \\
\hline 6 & 3 & 3 & 5 & 3 & 7215.2602 & -0.0134 \\
\hline 2 & 1 & 1 & 1 & 0 & 3482.8832 & 0.0015 \\
\hline 3 & 1 & 2 & 2 & 0 & 4821.9419 & -0.0004 \\
\hline 4 & 1 & 3 & 3 & 3 & 6218.6955 & 0.0020 \\
\hline 5 & 1 & 4 & 4 & 0 & 7683.8266 & 0.0058 \\
\hline 2 & 2 & 1 & 1 & 1 & 5291.6367 & 0.0050 \\
\hline 3 & 2 & 1 & 2 & 1 & 6331.7465 & -0.0024 \\
\hline 3 & 2 & 2 & 2 & 1 & 6580.8529 & -0.0026 \\
\hline 4 & 2 & 2 & 3 & 1 & 7451.5738 & -0.0054 \\
\hline 4 & 2 & 3 & 3 & 3 & 7916.1844 & 0.0054 \\
\hline 4 & 2 & 3 & 4 & 1 & 2395.8474 & -0.0026 \\
\hline 3 & 2 & 2 & 3 & 2 & 2575.6347 & -0.0022 \\
\hline 3 & 2 & 1 & 3 & 3 & 3178.5723 & -0.0026 \\
\hline 4 & 2 & 2 & 4 & 1 & 3443.9318 & -0.0036 \\
\hline 5 & 3 & 3 & 5 & 2 & 4584.2315 & -0.0093 \\
\hline 6 & 3 & 4 & 6 & 2 & 4433.6056 & 0.0053 \\
\hline 7 & 3 & 5 & 7 & 2 & 4220.3181 & 0.0073 \\
\hline
\end{tabular}


Table S35. Measured frequencies ( $\left.v_{\mathrm{obs}}\right)$ and residuals $\left(v_{\mathrm{obs}}-v_{\mathrm{cal}}\right)$ of the rotational transitions of the DOH isotopic species of isomer EQC-4 of limonene- $\mathrm{H}_{2} \mathrm{O}$.

\begin{tabular}{|c|c|c|c|c|c|c|c|}
\hline$J^{\prime}$ & $K_{-1}^{\prime}$ & $K_{+1}^{\prime}$ & $J^{\prime \prime}$ & $K^{\prime \prime}{ }_{-1}$ & $K^{\prime \prime}{ }_{+1}$ & $v_{\mathrm{obs}} / \mathrm{MHz}$ & $v_{\text {obs }}-v_{\text {calc }} / \mathrm{MHz}$ \\
\hline 3 & 1 & 3 & 2 & 1 & 2 & 3418.0786 & 0.0003 \\
\hline 3 & 0 & 3 & 2 & 0 & 2 & 3537.0071 & 0.0006 \\
\hline 3 & 1 & 2 & 2 & 1 & 1 & 3704.3119 & 0.0003 \\
\hline 4 & 1 & 4 & 3 & 1 & 3 & 4549.7342 & -0.0006 \\
\hline 4 & 0 & 4 & 3 & 0 & 3 & 4684.1702 & -0.0010 \\
\hline 4 & 1 & 3 & 3 & 1 & 2 & 4929.7684 & 0.0000 \\
\hline 5 & 1 & 5 & 4 & 1 & 4 & 5675.7432 & -0.0013 \\
\hline 5 & 0 & 5 & 4 & 0 & 4 & 5808.9361 & -0.0001 \\
\hline 5 & 1 & 4 & 4 & 1 & 3 & 6146.1875 & 0.0003 \\
\hline 6 & 1 & 6 & 5 & 1 & 5 & 6795.7661 & 0.0014 \\
\hline 6 & 0 & 6 & 5 & 0 & 5 & 6913.6366 & 0.0013 \\
\hline 6 & 1 & 5 & 5 & 1 & 4 & 7350.0489 & 0.0063 \\
\hline 7 & 1 & 7 & 6 & 1 & 6 & 7909.9494 & -0.0077 \\
\hline 3 & 2 & 2 & 2 & 2 & 1 & 3565.7099 & -0.0117 \\
\hline 3 & 2 & 1 & 2 & 2 & 0 & 3594.4543 & -0.0085 \\
\hline 4 & 2 & 3 & 3 & 2 & 2 & 4748.6406 & 0.0056 \\
\hline 4 & 2 & 2 & 3 & 2 & 1 & 4818.7672 & -0.0027 \\
\hline 5 & 2 & 4 & 4 & 2 & 3 & 5926.7396 & 0.0118 \\
\hline 5 & 2 & 3 & 4 & 2 & 2 & 6060.5560 & -0.0094 \\
\hline 6 & 2 & 5 & 5 & 2 & 4 & 7098.8891 & 0.0161 \\
\hline 6 & 2 & 4 & 5 & 2 & 3 & 7316.1732 & -0.0107 \\
\hline 4 & 3 & 1 & 3 & 3 & 0 & 4769.8278 & -0.0054 \\
\hline 5 & 3 & 3 & 4 & 3 & 2 & 5964.2831 & -0.0045 \\
\hline 5 & 3 & 2 & 4 & 3 & 1 & 5971.4696 & 0.0124 \\
\hline 6 & 3 & 4 & 5 & 3 & 3 & 7162.2406 & 0.0086 \\
\hline 6 & 3 & 3 & 5 & 3 & 2 & 7181.0574 & -0.0121 \\
\hline 2 & 1 & 1 & 1 & 0 & 1 & 3462.1769 & 0.0159 \\
\hline 3 & 1 & 2 & 2 & 0 & 2 & 4796.5600 & 0.0024 \\
\hline 4 & 1 & 3 & 3 & 0 & 3 & 6189.3190 & -0.0004 \\
\hline 5 & 1 & 4 & 4 & 0 & 4 & 7651.3341 & -0.0014 \\
\hline 2 & 2 & 1 & 1 & 1 & 1 & 5250.0219 & -0.0088 \\
\hline 3 & 2 & 2 & 2 & 1 & 2 & 6534.1182 & 0.0032 \\
\hline 4 & 2 & 2 & 3 & 1 & 2 & 7397.9779 & -0.0076 \\
\hline 4 & 2 & 3 & 3 & 1 & 3 & 7864.6776 & 0.0060 \\
\hline
\end{tabular}


Table S36. Measured frequencies ( $\left.v_{\text {obs }}\right)$ and residuals ( $v_{\text {obs }}-v_{\text {cal }}$ ) of the rotational transitions of the HOD isotopic species of isomer EQC-4 of limonene- $\mathrm{H}_{2} \mathrm{O}$.

\begin{tabular}{|c|c|c|c|c|c|c|}
\hline$J^{\prime}$ & $K_{-1}^{\prime}$ & $K^{\prime}+1$ & $J^{\prime \prime}$ & $K^{\prime \prime}{ }_{-1} K^{\prime \prime}+1$ & $v_{\text {obs }} / \mathrm{MHz}$ & $v_{\text {obs }}-v_{\text {calc }} / \mathrm{MHz}$ \\
\hline 3 & 1 & 3 & 2 & 1 & 3395.0264 & 0.0046 \\
\hline 3 & 0 & 3 & 2 & 0 & 3516.1406 & 0.0011 \\
\hline 3 & 1 & 2 & 2 & 1 & 3690.4166 & -0.0102 \\
\hline 4 & 1 & 4 & 3 & 1 & 4518.2790 & 0.0060 \\
\hline 4 & 0 & 4 & 3 & 0 & 4653.4293 & 0.0037 \\
\hline 4 & 1 & 3 & 3 & 1 & 4910.2425 & -0.0079 \\
\hline 5 & 1 & 5 & 4 & 1 & 5635.4260 & 0.0060 \\
\hline 5 & 0 & 5 & 4 & 0 & 5766.8812 & 0.0055 \\
\hline 5 & 1 & 4 & 4 & 1 & 6119.9917 & -0.0022 \\
\hline 6 & 1 & 6 & 5 & 1 & 6746.1697 & -0.0019 \\
\hline 6 & 0 & 6 & 5 & 0 & 6859.8365 & 0.0022 \\
\hline 6 & 1 & 5 & 5 & 1 & 7315.6811 & 0.0088 \\
\hline 7 & 0 & 7 & 6 & 0 & 7939.7224 & -0.0104 \\
\hline 3 & 2 & 2 & 2 & 2 & 3547.7183 & 0.0081 \\
\hline 3 & 2 & 1 & 2 & 2 & 3579.2952 & -0.0131 \\
\hline 4 & 2 & 3 & 3 & 2 & 4724.0515 & 0.0031 \\
\hline 4 & 2 & 2 & 3 & 2 & 4800.8992 & -0.0120 \\
\hline 5 & 2 & 4 & 4 & 2 & 5895.0913 & 0.0068 \\
\hline 5 & 2 & 3 & 4 & 2 & 6040.9212 & -0.0149 \\
\hline 6 & 2 & 5 & 5 & 2 & 7059.6066 & 0.0154 \\
\hline 6 & 2 & 4 & 5 & 2 & 7294.5434 & -0.0127 \\
\hline 5 & 3 & 3 & 4 & 3 & 5936.1974 & 0.0023 \\
\hline 5 & 3 & 2 & 4 & 3 & 5944.6038 & 0.0022 \\
\hline 6 & 3 & 4 & 5 & 3 & 7128.7312 & 0.0129 \\
\hline 6 & 3 & 3 & 5 & 3 & 7150.7459 & -0.0118 \\
\hline 5 & 1 & 4 & 4 & 0 & 7618.2403 & 0.0108 \\
\hline 2 & 2 & 0 & 1 & 1 & 5057.5262 & 0.0010 \\
\hline 3 & 2 & 1 & 2 & 1 & 6173.0586 & -0.0112 \\
\hline 3 & 2 & 2 & 2 & 1 & 6429.3189 & 0.0135 \\
\hline 4 & 2 & 2 & 3 & 1 & 7283.5394 & -0.0147 \\
\hline 4 & 2 & 3 & 3 & 1 & 7758.3372 & 0.0052 \\
\hline
\end{tabular}


Table S37. Measured frequencies ( $\left.v_{\text {obs }}\right)$ and residuals $\left(v_{\text {obs }}-v_{\text {cal }}\right.$ ) of the rotational transitions of the DOD isotopic species of isomer EQC-4 of limonene- $\mathrm{H}_{2} \mathrm{O}$.

\begin{tabular}{|c|c|c|c|c|c|c|}
\hline$J^{\prime}$ & $K_{-1}^{\prime}$ & $K^{\prime}+1$ & $J^{\prime \prime}$ & $K^{\prime \prime}{ }_{-1} K^{\prime \prime}{ }_{+1}$ & $v_{\mathrm{obs}} / \mathrm{MHz}$ & $v_{\text {obs }}-v_{\text {calc }} / \mathrm{MHz}$ \\
\hline 2 & 0 & 2 & 1 & $\begin{array}{ll}0 & 1\end{array}$ & 2345.2070 & -0.0039 \\
\hline 2 & 1 & 1 & 1 & 1 & 2452.6387 & -0.0065 \\
\hline 3 & 1 & 3 & 2 & 12 & 3376.2480 & 0.0018 \\
\hline 3 & 0 & 3 & 2 & 0 & 3497.7472 & -0.0010 \\
\hline 3 & 1 & 2 & 2 & 11 & 3673.6203 & -0.0050 \\
\hline 4 & 0 & 4 & 3 & 3 & 4628.1796 & 0.0009 \\
\hline 4 & 1 & 3 & 3 & 2 & 4887.5923 & -0.0021 \\
\hline 5 & 1 & 5 & 4 & 1 & 5603.6513 & 0.0026 \\
\hline 5 & 0 & 5 & 4 & 0 & 5734.4491 & 0.0075 \\
\hline 5 & 1 & 4 & 4 & 1 & 6091.2169 & 0.0002 \\
\hline 6 & 1 & 6 & 5 & 1 & 6707.7462 & 0.0006 \\
\hline 6 & 0 & 6 & 5 & 0 & 6820.1589 & 0.0053 \\
\hline 6 & 1 & 5 & 5 & 1 & 7280.3964 & 0.0086 \\
\hline 7 & 1 & 7 & 6 & 1 & 7805.6414 & -0.0042 \\
\hline 7 & 0 & 7 & 6 & 0 & 7893.0068 & -0.0014 \\
\hline 4 & 2 & 3 & 3 & 2 & 4700.3464 & 0.0032 \\
\hline 4 & 2 & 2 & 3 & 2 & 4778.8795 & -0.0079 \\
\hline 5 & 2 & 4 & 4 & 2 & 5865.2297 & 0.0068 \\
\hline 5 & 2 & 3 & 4 & 2 & 6014.0341 & -0.0103 \\
\hline 6 & 2 & 5 & 5 & 2 & 7023.4173 & -0.0088 \\
\hline 6 & 2 & 4 & 5 & 3 & 7262.6810 & -0.0074 \\
\hline 5 & 3 & 3 & 4 & 3 & 5907.2307 & 0.0035 \\
\hline 5 & 3 & 2 & 4 & 3 & 5915.9651 & 0.0002 \\
\hline 6 & 3 & 4 & 5 & 3 & 7094.0068 & 0.0108 \\
\hline 6 & 3 & 3 & 5 & 3 & 7116.8828 & -0.0083 \\
\hline 2 & 1 & 1 & 1 & 0 & 3404.9056 & -0.0011 \\
\hline 3 & 1 & 2 & 2 & 0 & 4733.3194 & -0.0016 \\
\hline 4 & 1 & 3 & 3 & 0 & 6123.1748 & 0.0076 \\
\hline 5 & 1 & 4 & 4 & 0 & 7586.2097 & 0.0046 \\
\hline 2 & 2 & 0 & 1 & 1 & 5019.7742 & -0.0044 \\
\hline 2 & 2 & 1 & 1 & 1 & 5110.8748 & -0.0040 \\
\hline 3 & 2 & 2 & 2 & 1 & 6386.8145 & 0.0038 \\
\hline 4 & 2 & 2 & 3 & 1 & 7234.7474 & -0.0040 \\
\hline 4 & 2 & 3 & 3 & 3 & 7710.9113 & 0.0036 \\
\hline
\end{tabular}


Table S38. Measured frequencies ( $v_{\text {obs }}$ ) and residuals ( $v_{\text {obs }}-v_{\text {cal }}$ ) of the rotational transitions of the $\mathrm{H}_{2}{ }^{18} \mathrm{O}$ isotopic species of isomer EQC-4 of limonene- $\mathrm{H}_{2} \mathrm{O}$.

\begin{tabular}{|c|c|c|c|c|c|c|}
\hline$J^{\prime}$ & $K_{-1}^{\prime}$ & $K^{\prime}+1$ & $J^{\prime \prime}$ & $K^{\prime \prime}{ }_{-1} K^{\prime \prime}+1$ & $v_{\mathrm{obs}} / \mathrm{MHz}$ & $v_{\text {obs }}-v_{\text {calc }} / \mathrm{MHz}$ \\
\hline 2 & 1 & 2 & 1 & 1 & 2258.2892 & 0.0073 \\
\hline 2 & 0 & 2 & 1 & 0 & 2349.6921 & -0.0022 \\
\hline 2 & 1 & 1 & 1 & 10 & 2457.7207 & 0.0012 \\
\hline 3 & 1 & 3 & 2 & 2 & 3382.4231 & -0.0010 \\
\hline 3 & 0 & 3 & 2 & 0 & 3504.1328 & -0.0027 \\
\hline 3 & 1 & 2 & 2 & 1 & 3681.1415 & -0.0004 \\
\hline 4 & 1 & 4 & 3 & 13 & 4501.1550 & 0.0000 \\
\hline 4 & 0 & 4 & 3 & 3 & 4636.1440 & -0.0003 \\
\hline 5 & 1 & 5 & 4 & 1 & 5613.5811 & -0.0004 \\
\hline 5 & 0 & 5 & 4 & 0 & 5743.7428 & -0.0020 \\
\hline 5 & 1 & 4 & 4 & 1 & 6103.1473 & 0.0006 \\
\hline 6 & 1 & 6 & 5 & 1 & 6719.4394 & 0.0003 \\
\hline 6 & 0 & 6 & 5 & 0 & 6830.7691 & -0.0013 \\
\hline 6 & 1 & 5 & 5 & 1 & 7294.1297 & 0.0082 \\
\hline 7 & 1 & 7 & 6 & 1 & 7819.0476 & -0.0012 \\
\hline 7 & 0 & 7 & 6 & 0 & 7905.1140 & -0.0003 \\
\hline 3 & 2 & 2 & 2 & 2 & 3536.9832 & 0.0090 \\
\hline 3 & 2 & 1 & 2 & 2 & 3569.8321 & -0.0081 \\
\hline 4 & 2 & 3 & 3 & 2 & 4709.4804 & 0.0018 \\
\hline 4 & 2 & 2 & 3 & 1 & 4789.2983 & -0.0069 \\
\hline 5 & 2 & 4 & 4 & 3 & 5876.4732 & 0.0073 \\
\hline 5 & 2 & 3 & 4 & 2 & 6027.5310 & -0.0076 \\
\hline 6 & 2 & 5 & 5 & 2 & 7036.6715 & 0.0074 \\
\hline 6 & 2 & 4 & 5 & 2 & 7279.1444 & -0.0092 \\
\hline 5 & 3 & 3 & 4 & 2 & 5919.1477 & 0.0047 \\
\hline 5 & 3 & 2 & 4 & 3 & 5928.1444 & 0.0017 \\
\hline 6 & 3 & 4 & 5 & 3 & 7108.3212 & 0.0005 \\
\hline 6 & 3 & 3 & 5 & 3 & 7131.8850 & -0.0064 \\
\hline 2 & 1 & 1 & 1 & 0 & 3402.8770 & -0.0037 \\
\hline 3 & 1 & 2 & 2 & 0 & 4734.3291 & 0.0009 \\
\hline 4 & 1 & 3 & 3 & 0 & 6127.6133 & -0.0004 \\
\hline 5 & 1 & 4 & 4 & 0 & 7594.6212 & 0.0052 \\
\hline 2 & 2 & 0 & 1 & 1 & 5002.3432 & 0.0015 \\
\hline 2 & 2 & 1 & 1 & 1 & 5093.7498 & -0.0009 \\
\hline 3 & 2 & 1 & 2 & 1 & 6114.4608 & -0.0015 \\
\hline 3 & 2 & 2 & 2 & 12 & 6372.4431 & 0.0001 \\
\hline 4 & 2 & 2 & 3 & 1 & 7222.6216 & -0.0040 \\
\hline 4 & 2 & 3 & 3 & 1 & 7699.5002 & 0.0026 \\
\hline
\end{tabular}


Table S39. Measured frequencies ( $\left.v_{\text {obs }}\right)$ and residuals ( $v_{\text {obs }}-v_{\text {cal }}$ ) of the rotational transitions of the parent species of isomer EQC-2 of limonene- $\mathrm{H}_{2} \mathrm{O}$.

\begin{tabular}{|c|c|c|c|c|c|c|}
\hline$J^{\prime}$ & $K^{\prime}-1$ & $K^{\prime}+1$ & $J^{\prime \prime}$ & $K^{\prime \prime}{ }_{-1} K^{\prime \prime}+1$ & $v_{\mathrm{obs}} / \mathrm{MHz}$ & $v_{\text {obs }}-v_{\text {calc }} / \mathrm{MHz}$ \\
\hline 3 & 1 & 3 & 2 & 2 & 3176.0027 & -0.0029 \\
\hline 3 & 0 & 3 & 2 & 2 & 3294.1795 & -0.0042 \\
\hline 3 & 1 & 2 & 2 & 1 & 3452.8032 & -0.0013 \\
\hline 4 & 1 & 4 & 3 & 1 & 4228.1607 & -0.0028 \\
\hline 4 & 0 & 4 & 3 & 3 & 4365.2879 & -0.0054 \\
\hline 5 & 1 & 5 & 4 & 1 & 5275.4318 & -0.0044 \\
\hline 5 & 0 & 5 & 4 & 4 & 5416.4914 & -0.0009 \\
\hline 5 & 1 & 4 & 4 & 1 & 5732.0089 & -0.0091 \\
\hline 6 & 1 & 6 & 5 & 5 & 6317.3951 & -0.0015 \\
\hline 6 & 0 & 6 & 5 & 5 & 6448.3744 & -0.0096 \\
\hline 6 & 1 & 5 & 5 & 4 & 6857.8578 & -0.0115 \\
\hline 7 & 1 & 7 & 6 & 6 & 7354.0336 & 0.0149 \\
\hline 7 & 0 & 7 & 6 & 6 & 7465.2260 & -0.0026 \\
\hline 3 & 2 & 2 & 2 & 2 & 3318.1856 & 0.0058 \\
\hline 3 & 2 & 1 & 2 & 2 & 3342.1945 & 0.0033 \\
\hline 4 & 2 & 3 & 3 & 2 & 4419.5243 & 0.0038 \\
\hline 4 & 2 & 2 & 3 & 2 & 4478.4659 & -0.0006 \\
\hline 5 & 2 & 4 & 4 & 2 & 5516.8378 & 0.0026 \\
\hline 5 & 2 & 3 & 4 & 2 & 5630.5661 & 0.0005 \\
\hline 7 & 2 & 6 & 6 & 2 & 7695.6247 & 0.0035 \\
\hline 2 & 1 & 2 & 1 & 0 & 3130.1337 & 0.0052 \\
\hline 3 & 1 & 3 & 2 & 0 & 4100.0569 & 0.0118 \\
\hline 5 & 1 & 5 & 4 & 0 & 5944.1701 & 0.0023 \\
\hline 2 & 1 & 1 & 1 & 0 & 3407.1857 & 0.0104 \\
\hline 3 & 1 & 2 & 2 & 0 & 4653.8990 & 0.0081 \\
\hline 5 & 1 & 4 & 4 & 0 & 7322.5093 & 0.0018 \\
\hline 5 & 0 & 5 & 4 & 1 & 4747.7667 & 0.0061 \\
\hline 4 & 2 & 2 & 3 & 1 & 7404.9152 & -0.0126 \\
\hline 3 & 2 & 2 & 2 & 1 & 6626.2579 & 0.0020 \\
\hline 3 & 0 & 3 & 2 & 1 & 2370.1453 & 0.0011 \\
\hline
\end{tabular}

Table S40. Measured frequencies ( $v_{\text {obs }}$ ) and residuals ( $v_{\text {obs }}-v_{\text {cal }}$ ) of the rotational transitions of the DOH isotopic species of isomer EQC-2 of limonene- $\mathrm{H}_{2} \mathrm{O}$.

\begin{tabular}{cccccccc}
\hline$J^{\prime}$ & $K^{\prime}{ }_{-1} K^{\prime}{ }_{+1}$ & $J^{\prime \prime}$ & $K^{\prime \prime}{ }_{-1} K^{\prime \prime}{ }_{+1}$ & $v_{\mathrm{obs}} / \mathrm{MHz}$ & \multicolumn{1}{c}{$v_{\mathrm{obs}}-v_{\text {calc }} / \mathrm{MHz}$} \\
\hline 3 & 1 & 3 & 2 & 1 & 2 & 3145.1409 & -0.0102 \\
3 & 0 & 3 & 2 & 0 & 2 & 3263.0671 & -0.0038 \\
3 & 1 & 2 & 2 & 1 & 1 & 3421.4595 & 0.0089 \\
4 & 0 & 4 & 3 & 0 & 3 & 4323.8049 & 0.0017 \\
4 & 1 & 3 & 3 & 1 & 2 & 4554.2723 & 0.0067 \\
5 & 1 & 5 & 4 & 1 & 4 & 5224.0061 & -0.0026 \\
5 & 0 & 5 & 4 & 0 & 4 & 5364.6318 & -0.0010 \\
5 & 1 & 4 & 4 & 1 & 3 & 5679.7460 & 0.0004 \\
6 & 1 & 6 & 5 & 1 & 5 & 6255.6924 & 0.0078 \\
6 & 0 & 6 & 5 & 0 & 5 & 6386.1899 & 0.0066 \\
6 & 1 & 5 & 5 & 1 & 4 & 6795.1115 & -0.0130 \\
7 & 1 & 7 & 6 & 1 & 6 & 7282.0313 & 0.0044 \\
7 & 0 & 7 & 6 & 0 & 6 & 7392.7397 & 0.0050 \\
4 & 2 & 3 & 3 & 2 & 2 & 4378.0510 & 0.0004 \\
4 & 2 & 2 & 3 & 2 & 1 & 4437.0199 & 0.0041 \\
5 & 2 & 4 & 4 & 2 & 3 & 5465.0012 & 0.0070 \\
6 & 2 & 5 & 5 & 2 & 4 & 6546.9614 & 0.0099 \\
6 & 2 & 4 & 5 & 2 & 3 & 6734.5696 & -0.0071 \\
6 & 3 & 3 & 5 & 3 & 2 & 6614.4304 & -0.0004 \\
6 & 3 & 4 & 5 & 3 & 3 & 6600.7752 & -0.0018 \\
5 & 1 & 5 & 4 & 0 & 4 & 5889.3355 & 0.0030 \\
6 & 1 & 6 & 5 & 0 & 5 & 6780.3793 & -0.0051 \\
7 & 1 & 7 & 6 & 0 & 6 & 7676.2179 & -0.0100 \\
4 & 0 & 4 & 3 & 1 & 3 & 3521.6857 & -0.0129 \\
5 & 0 & 5 & 4 & 1 & 4 & 4699.3102 & 0.0013 \\
2 & 2 & 1 & 1 & 1 & 0 & 5302.0557 & -0.0012
\end{tabular}


Table S41. Measured frequencies ( $\left.v_{\text {obs }}\right)$ and residuals ( $v_{\text {obs }}-v_{\text {cal }}$ ) of the rotational transitions of the HOD isotopic species of isomer EQC-2 of limonene- $\mathrm{H}_{2} \mathrm{O}$.

\begin{tabular}{rrrrrrrrr}
\hline$J^{\prime}$ & $K^{\prime}{ }_{-1}$ & $K^{\prime}{ }_{+1}$ & $J^{\prime \prime}$ & $K^{\prime \prime}{ }_{-1} K^{\prime \prime}{ }_{1}$ & $v_{\text {obs }} / \mathrm{MHz}$ & \multicolumn{1}{c}{$v_{\text {obs }}-v_{\text {calc }} / \mathrm{MHz}$} \\
\hline 3 & 1 & 3 & 2 & 1 & 2 & 3126.3045 & -0.0106 \\
3 & 0 & 3 & 2 & 0 & 2 & 3245.0455 & -0.0023 \\
3 & 1 & 2 & 2 & 1 & 1 & 3406.5034 & 0.0083 \\
4 & 1 & 4 & 3 & 1 & 3 & 4161.5588 & -0.0050 \\
4 & 0 & 4 & 3 & 0 & 3 & 4298.3506 & -0.0082 \\
4 & 1 & 3 & 3 & 1 & 2 & 4533.8703 & 0.0058 \\
5 & 1 & 5 & 4 & 1 & 4 & 5191.6930 & -0.0072 \\
5 & 0 & 5 & 4 & 0 & 4 & 5330.9731 & -0.0042 \\
5 & 1 & 4 & 4 & 1 & 3 & 5653.4298 & 0.0016 \\
6 & 1 & 6 & 5 & 1 & 5 & 6216.3239 & 0.0089 \\
6 & 0 & 6 & 5 & 0 & 5 & 6343.9486 & 0.0012 \\
6 & 1 & 5 & 5 & 1 & 4 & 6762.2078 & -0.0053 \\
7 & 1 & 7 & 6 & 1 & 6 & 7235.4253 & -0.0042 \\
7 & 0 & 7 & 6 & 0 & 6 & 7342.1310 & 0.0033 \\
7 & 1 & 6 & 6 & 1 & 5 & 7856.7011 & -0.0109 \\
4 & 2 & 3 & 3 & 2 & 2 & 4355.5510 & 0.0107 \\
4 & 2 & 2 & 3 & 2 & 1 & 4417.6885 & -0.0197 \\
5 & 2 & 4 & 4 & 2 & 3 & 5436.4507 & 0.0200 \\
6 & 2 & 5 & 5 & 2 & 4 & 6512.0738 & 0.0142 \\
7 & 2 & 6 & 6 & 2 & 5 & 7581.4967 & -0.0006
\end{tabular}

Table S42. Measured frequencies ( $\left.v_{\text {obs }}\right)$ and residuals ( $v_{\text {obs }}-v_{\text {cal }}$ ) of the rotational transitions of the DOD isotopic species of isomer EQC-2 of limonene- $\mathrm{H}_{2} \mathrm{O}$.

\begin{tabular}{|c|c|c|c|c|c|c|}
\hline$J^{\prime}$ & $K_{-1}^{\prime}$ & $K_{+1}^{\prime}$ & $J^{\prime \prime}$ & $K^{\prime \prime}{ }_{-1} K^{\prime \prime}{ }_{+1}$ & $v_{\text {obs }} / \mathrm{MHz}$ & $v_{\text {obs }}-v_{\text {calc }} / \mathrm{MHz}$ \\
\hline 3 & 1 & 3 & 2 & 2 & 3096.5757 & -0.0038 \\
\hline 3 & 0 & 3 & 2 & 2 & 3214.9134 & -0.0004 \\
\hline 3 & 1 & 2 & 2 & 11 & 3375.7973 & 0.0090 \\
\hline 4 & 1 & 4 & 3 & 3 & 4121.9391 & -0.0042 \\
\hline 4 & 0 & 4 & 3 & 3 & 4258.2942 & -0.0012 \\
\hline 5 & 1 & 5 & 4 & 4 & 5142.2103 & -0.0041 \\
\hline 5 & 0 & 5 & 4 & 4 & 5281.0595 & -0.0048 \\
\hline 5 & 1 & 4 & 4 & 3 & 5602.3513 & 0.0020 \\
\hline 6 & 1 & 6 & 5 & 1 & 6156.9852 & 0.0010 \\
\hline 6 & 0 & 6 & 5 & 5 & 6284.2541 & 0.0006 \\
\hline 6 & 1 & 5 & 5 & 4 & 6701.0043 & -0.0060 \\
\hline 7 & 1 & 7 & 6 & 6 & 7166.2742 & 0.0012 \\
\hline 7 & 0 & 7 & 6 & 6 & 7272.6983 & 0.0030 \\
\hline 7 & 1 & 6 & 6 & 5 & 7785.4464 & -0.0004 \\
\hline 4 & 2 & 3 & 3 & 2 & 4315.2667 & 0.0169 \\
\hline 4 & 2 & 2 & 3 & 2 & 4377.1610 & 0.0005 \\
\hline 5 & 2 & 4 & 4 & 3 & 5386.0998 & 0.0007 \\
\hline 5 & 2 & 3 & 4 & 2 & 5505.2056 & 0.0003 \\
\hline 6 & 2 & 5 & 5 & 2 & 6451.7087 & 0.0013 \\
\hline 6 & 2 & 4 & 5 & 3 & 6647.3617 & -0.0112 \\
\hline 2 & 1 & 2 & 1 & 1 & 3041.7208 & 0.0160 \\
\hline 3 & 1 & 3 & 2 & 2 & 3984.5179 & -0.0102 \\
\hline 5 & 1 & 5 & 4 & 0 & 5775.4740 & -0.0027 \\
\hline 6 & 1 & 6 & 5 & 5 & 6651.3898 & -0.0068 \\
\hline 7 & 1 & 7 & 6 & 0 & 7533.4227 & 0.0066 \\
\hline 4 & 0 & 4 & 3 & 3 & 3488.6840 & 0.0030 \\
\hline 5 & 0 & 5 & 4 & 1 & 4647.7953 & -0.0067 \\
\hline 6 & 0 & 6 & 5 & 1 & 5789.8503 & 0.0092 \\
\hline
\end{tabular}


Table S43. Measured frequencies ( $v_{\text {obs }}$ ) and residuals ( $v_{\text {obs }}-v_{\text {cal }}$ ) of the rotational transitions of the $\mathrm{H}_{2}{ }^{18} \mathrm{O}$ isotopic species of isomer EQC-2 of limonene- $\mathrm{H}_{2} \mathrm{O}$.

\begin{tabular}{rrrrrrrr}
\hline$J^{\prime}$ & $K^{\prime}{ }_{-1} K^{\prime}{ }_{+1} J^{\prime \prime}$ & $K^{\prime \prime}{ }_{-1} K^{\prime \prime}{ }_{+1}$ & $V_{\text {obs }} / \mathrm{MHz}$ & \multicolumn{1}{c}{$v_{\text {obs }}-v_{\text {calc }} / \mathrm{MHz}$} \\
\hline 4 & 1 & 4 & 3 & 1 & 3 & 4136.1478 & 0.0018 \\
4 & 0 & 4 & 3 & 0 & 3 & 4272.4867 & -0.0072 \\
4 & 1 & 3 & 3 & 1 & 2 & 4508.8653 & 0.0043 \\
3 & 1 & 3 & 2 & 1 & 2 & 3107.3195 & 0.0009 \\
3 & 0 & 3 & 2 & 0 & 2 & 3225.9220 & -0.0008 \\
3 & 1 & 2 & 2 & 1 & 1 & 3387.8373 & 0.0020 \\
5 & 1 & 5 & 4 & 1 & 4 & 5159.8180 & 0.0132 \\
5 & 0 & 5 & 4 & 0 & 4 & 5298.1958 & 0.0052 \\
5 & 1 & 4 & 4 & 1 & 3 & 5621.9400 & -0.0122 \\
6 & 1 & 6 & 5 & 1 & 5 & 6177.8935 & 0.0010 \\
6 & 0 & 6 & 5 & 0 & 5 & 6304.1837 & -0.0115 \\
7 & 1 & 7 & 6 & 1 & 6 & 7190.4515 & 0.0061 \\
7 & 0 & 7 & 6 & 0 & 6 & 7295.5229 & -0.0140 \\
4 & 2 & 2 & 3 & 2 & 1 & 4393.4397 & -0.0057 \\
5 & 2 & 4 & 4 & 2 & 3 & 5404.9580 & 0.0132 \\
5 & 2 & 3 & 4 & 2 & 2 & 5526.0446 & 0.0061 \\
6 & 2 & 5 & 5 & 2 & 4 & 6474.1187 & 0.0044 \\
6 & 2 & 4 & 5 & 2 & 3 & 6672.7563 & -0.0095 \\
7 & 2 & 6 & 6 & 2 & 5 & 7537.0219 & 0.0110 \\
3 & 1 & 3 & 2 & 0 & 2 & 3985.6976 & -0.0059 \\
5 & 1 & 5 & 4 & 0 & 4 & 5783.2340 & -0.0035 \\
3 & 1 & 2 & 2 & 0 & 2 & 4547.0108 & -0.0072 \\
4 & 2 & 2 & 3 & 1 & 2 & 7185.9473 & 0.0028 \\
3 & 2 & 2 & 2 & 1 & 2 & 6428.9378 & 0.0035
\end{tabular}

Table S44. Measured frequencies ( $v_{\text {obs }}$ ) and residuals ( $v_{\text {obs }}-v_{c a l}$ ) of the rotational transitions of the parent species of isomer EQa-4 of limonene- $\mathrm{H}_{2} \mathrm{O}$.

\begin{tabular}{ccccccccc}
\hline$J^{\prime}$ & $K^{\prime}{ }_{-1}$ & $K^{\prime}{ }_{+1}$ & $J^{\prime \prime}$ & $K^{\prime \prime}{ }_{-1} K^{\prime \prime}{ }_{1}$ & $v_{\mathrm{obs}} / \mathrm{MHz}$ & \multicolumn{1}{c}{$v_{\text {obs }}-v_{\text {calc }} / \mathrm{MHz}$} \\
\hline 2 & 1 & 1 & 1 & 1 & 0 & 2390.6958 & -0.0113 \\
3 & 1 & 3 & 2 & 1 & 2 & 3475.8686 & -0.0063 \\
3 & 0 & 3 & 2 & 0 & 2 & 3527.1965 & -0.0014 \\
3 & 1 & 2 & 2 & 1 & 1 & 3585.4068 & 0.0026 \\
4 & 1 & 4 & 3 & 1 & 3 & 4633.3384 & 0.0059 \\
4 & 0 & 4 & 3 & 0 & 3 & 4698.1761 & -0.0069 \\
4 & 1 & 3 & 3 & 1 & 2 & 4779.2893 & 0.0013 \\
5 & 1 & 5 & 4 & 1 & 4 & 5789.8428 & 0.0006 \\
5 & 0 & 5 & 4 & 0 & 4 & 5865.2155 & 0.0040 \\
5 & 1 & 4 & 4 & 1 & 3 & 5972.0594 & 0.0144 \\
6 & 1 & 5 & 5 & 1 & 4 & 7163.3156 & -0.0090 \\
4 & 2 & 3 & 3 & 2 & 2 & 4707.5442 & 0.0032 \\
5 & 2 & 4 & 4 & 2 & 3 & 5883.1204 & -0.0034
\end{tabular}


Table S45. Measured frequencies ( $v_{\text {obs }}$ ) and residuals ( $v_{\text {obs }}-v_{c a l}$ ) of the rotational transitions of the parent species of isomer EQC-3 $\beta$ of limonene- $\mathrm{H}_{2} \mathrm{O}$.

\begin{tabular}{cccccccr}
\hline$J^{\prime}$ & $K_{-1}^{\prime}$ & $K^{\prime}{ }_{+1}$ & $J^{\prime \prime}$ & $K^{\prime \prime}{ }_{-1} K^{\prime \prime}{ }_{+1}$ & $v_{\text {obs }} / \mathrm{MHz}$ & \multicolumn{1}{c}{$v_{\text {obs }}-v_{\text {calc }} / \mathrm{MHz}$} \\
\hline 2 & 1 & 2 & 1 & 0 & 1 & 3264.9786 & -0.0016 \\
3 & 1 & 3 & 2 & 0 & 2 & 4329.0116 & -0.0004 \\
4 & 1 & 4 & 3 & 0 & 3 & 5371.5758 & 0.0008 \\
5 & 1 & 5 & 4 & 0 & 4 & 6396.0559 & 0.0085 \\
4 & 0 & 4 & 3 & 1 & 3 & 3600.2695 & -0.0082 \\
5 & 0 & 5 & 4 & 1 & 4 & 4803.8961 & -0.0073 \\
6 & 0 & 6 & 5 & 1 & 5 & 6012.0732 & -0.0052 \\
7 & 0 & 7 & 6 & 1 & 6 & 7218.8643 & -0.0024 \\
2 & 2 & 1 & 1 & 1 & 0 & 5444.4361 & -0.0150 \\
2 & 2 & 0 & 1 & 1 & 1 & 5494.5178 & -0.0037 \\
3 & 2 & 2 & 2 & 1 & 1 & 6532.0571 & 0.0135 \\
3 & 2 & 1 & 2 & 1 & 2 & 6685.5427 & 0.0013 \\
4 & 2 & 3 & 3 & 1 & 2 & 7595.1656 & -0.0094 \\
4 & 2 & 2 & 3 & 1 & 3 & 7910.2854 & 0.0038 \\
2 & 1 & 1 & 1 & 0 & 1 & 3410.2426 & 0.0003 \\
3 & 1 & 2 & 2 & 0 & 2 & 4619.5030 & 0.0017 \\
4 & 1 & 3 & 3 & 0 & 3 & 5855.5409 & 0.0045 \\
5 & 1 & 4 & 4 & 0 & 4 & 7121.3774 & 0.0015 \\
2 & 2 & 0 & 1 & 1 & 0 & 5446.1058 & 0.0050 \\
3 & 2 & 1 & 2 & 1 & 1 & 6540.2799 & 0.0007 \\
4 & 2 & 2 & 3 & 1 & 2 & 7619.7932 & 0.0008 \\
4 & 2 & 3 & 3 & 1 & 3 & 7885.6622 & -0.0020 \\
6 & 1 & 5 & 5 & 2 & 4 & 4106.7470 & 0.0115 \\
7 & 1 & 6 & 6 & 2 & 5 & 5395.7093 & -0.0083 \\
8 & 1 & 7 & 7 & 2 & 6 & 6700.6852 & 0.0044 \\
4 & 0 & 4 & 3 & 1 & 2 & 3309.7760 & -0.0124 \\
5 & 0 & 5 & 4 & 1 & 3 & 4319.9460 & 0.0041 \\
7 & 0 & 7 & 6 & 1 & 5 & 6204.9841 & 0.0119 \\
9 & 0 & 9 & 8 & 1 & 7 & 7880.8424 & -0.0052 \\
& & & & & & \\
\hline
\end{tabular}

Table S46. Measured frequencies ( $v_{\text {obs }}$ ) and residuals ( $v_{\text {obs }}-v_{\text {cal }}$ ) of the rotational transitions of the DOH isotopic species of isomer EQC-3 $\beta$ of limonene- $\mathrm{H}_{2} \mathrm{O}$.

\begin{tabular}{cccccccr}
\hline$J^{\prime}$ & $K^{\prime}{ }_{-1}$ & $K^{\prime}{ }_{+1}$ & $J^{\prime \prime}$ & $K^{\prime \prime}{ }_{-1} K^{\prime \prime}{ }_{+1}$ & $v_{\text {obs }} / \mathrm{MHz}$ & \multicolumn{1}{c}{$v_{\text {obs }}-v_{\text {calc }} / \mathrm{MHz}$} \\
\hline 3 & 1 & 3 & 2 & 0 & 2 & 4300.5992 & 0.0179 \\
4 & 1 & 4 & 3 & 0 & 3 & 5337.2150 & -0.0124 \\
5 & 1 & 5 & 4 & 0 & 4 & 6355.8928 & -0.0073 \\
6 & 1 & 6 & 5 & 0 & 5 & 7361.1222 & 0.0026 \\
4 & 0 & 4 & 3 & 1 & 3 & 3584.0350 & -0.0068 \\
5 & 0 & 5 & 4 & 1 & 4 & 4780.8385 & 0.0013 \\
6 & 0 & 6 & 5 & 1 & 5 & 5982.0940 & 0.0106 \\
7 & 0 & 7 & 6 & 1 & 6 & 7181.8578 & -0.0047 \\
3 & 1 & 2 & 2 & 0 & 2 & 4589.6821 & -0.0060 \\
4 & 1 & 3 & 3 & 0 & 3 & 5818.8870 & 0.0031 \\
2 & 2 & 0 & 1 & 1 & 0 & 5403.5108 & -0.0095 \\
3 & 2 & 1 & 2 & 1 & 1 & 6491.5546 & 0.0037 \\
3 & 2 & 1 & 2 & 1 & 2 & 6636.1126 & -0.0094 \\
3 & 2 & 2 & 2 & 1 & 1 & 6483.3327 & 0.0160
\end{tabular}


Table S47. Measured frequencies ( $\left.v_{\text {obs }}\right)$ and residuals ( $v_{\text {obs }}-v_{\text {cal }}$ ) of the rotational transitions of the HOD isotopic species of isomer EQC-3 $\beta$ of limonene- $\mathrm{H}_{2} \mathrm{O}$.

\begin{tabular}{|c|c|c|c|c|c|c|}
\hline$J^{\prime}$ & $K_{-1}^{\prime}$ & $K^{\prime}+1$ & $J^{\prime \prime}$ & $K^{\prime \prime}{ }_{-1} K^{\prime \prime}{ }_{+1}$ & $v_{\text {obs }} / \mathrm{MHz}$ & $\nu_{\text {obs }}-v_{\text {calc }} / \mathrm{MHz}$ \\
\hline 2 & 1 & 2 & 1 & 0 & 3196.1633 & -0.0092 \\
\hline 3 & 1 & 3 & 2 & 2 & 4243.6141 & 0.0127 \\
\hline 4 & 1 & 4 & 3 & 0 & 5268.7876 & -0.0090 \\
\hline 5 & 1 & 5 & 4 & 4 & 6275.6039 & -0.0013 \\
\hline 6 & 1 & 6 & 5 & 5 & 7269.1396 & -0.0019 \\
\hline 4 & 0 & 4 & 3 & 1 & 3589.9090 & -0.0030 \\
\hline 5 & 0 & 5 & 4 & 4 & 4780.9674 & 0.0036 \\
\hline 6 & 0 & 6 & 5 & 5 & 5975.2389 & 0.0013 \\
\hline 3 & 1 & 2 & 2 & 2 & 4547.9369 & -0.0007 \\
\hline 4 & 1 & 3 & 3 & 3 & 5775.7938 & 0.0023 \\
\hline 2 & 2 & 0 & 1 & 0 & 5302.0559 & -0.0074 \\
\hline 3 & 2 & 1 & 2 & 1 & 6381.6203 & 0.0028 \\
\hline 4 & 2 & 2 & 3 & 1 & 7446.6677 & 0.0037 \\
\hline 4 & 2 & 3 & 3 & 1 & 7722.9729 & -0.0106 \\
\hline 2 & 2 & 0 & 1 & 1 & 5352.7982 & 0.0050 \\
\hline 4 & 2 & 2 & 3 & 3 & 7751.0003 & 0.0001 \\
\hline 3 & 2 & 2 & 2 & 1 & 6372.2389 & 0.0003 \\
\hline 4 & 2 & 3 & 3 & 1 & 7418.6564 & 0.0090 \\
\hline
\end{tabular}

Table S48. Measured frequencies ( $\left.v_{\text {obs }}\right)$ and residuals $\left(v_{\text {obs }}-v_{\text {cal }}\right.$ ) of the rotational transitions of the DOD isotopic species of isomer EQC-3 $\beta$ of limonene- $\mathrm{H}_{2} \mathrm{O}$.

\begin{tabular}{cccccccc}
\hline$J^{\prime}$ & $K_{-1}^{\prime}$ & $K_{+1}^{\prime}$ & $J^{\prime \prime}$ & $K^{\prime \prime}{ }_{-1} K^{\prime \prime}{ }_{+1}$ & $v_{\text {obs }} / \mathrm{MHz}$ & $v_{\text {obs }}-v_{\text {calc }} / \mathrm{MHz}$ \\
\hline 2 & 1 & 1 & 1 & 0 & 1 & 3326.9713 & 0.0109 \\
3 & 1 & 2 & 2 & 0 & 2 & 4520.0489 & 0.0038 \\
4 & 1 & 3 & 3 & 0 & 3 & 5741.3177 & 0.0012 \\
5 & 1 & 4 & 4 & 0 & 4 & 6994.1828 & -0.0068 \\
4 & 1 & 4 & 3 & 0 & 3 & 5236.2244 & -0.0074 \\
5 & 1 & 5 & 4 & 0 & 4 & 6237.3261 & -0.0003 \\
6 & 1 & 6 & 5 & 0 & 5 & 7225.2672 & 0.0119 \\
4 & 0 & 4 & 3 & 1 & 3 & 3573.1993 & 0.0116 \\
5 & 0 & 5 & 4 & 1 & 4 & 4757.6268 & 0.0019 \\
6 & 0 & 6 & 5 & 1 & 5 & 5945.1807 & -0.0027 \\
7 & 0 & 7 & 6 & 1 & 6 & 7129.4700 & 0.0003 \\
4 & 0 & 4 & 3 & 1 & 2 & 3269.9827 & -0.0142 \\
5 & 0 & 5 & 4 & 1 & 3 & 4252.5441 & 0.0039 \\
6 & 0 & 6 & 5 & 1 & 4 & 5188.3188 & -0.0015 \\
7 & 0 & 7 & 6 & 1 & 5 & 6071.8069 & -0.0025 \\
3 & 2 & 1 & 2 & 1 & 2 & 6488.8126 & 0.0004 \\
4 & 2 & 2 & 3 & 1 & 3 & 7699.5309 & -0.0058
\end{tabular}


Table S49. Measured frequencies ( $v_{\text {obs }}$ ) and residuals ( $v_{\text {obs }}-v_{\text {cal }}$ ) of the rotational transitions of the $\mathrm{H}_{2}{ }^{18} \mathrm{O}$ isotopic species of isomer EQC-3 $\beta$ of limonene- $\mathrm{H}_{2} \mathrm{O}$.

\begin{tabular}{cccccccc}
\hline$J^{\prime}$ & $K^{\prime}{ }_{-1} K^{\prime}{ }_{+1}$ & $J^{\prime \prime}$ & $K^{\prime \prime}{ }_{-1} K^{\prime \prime}{ }_{+1}$ & $v_{\mathrm{obs}} / \mathrm{MHz}$ & \multicolumn{1}{c}{$\nu_{\text {obs }}-v_{\text {calc }} / \mathrm{MHz}$} \\
\hline 2 & 1 & 2 & 1 & 0 & 1 & 3170.2884 & -0.0004 \\
3 & 1 & 3 & 2 & 0 & 2 & 4208.0980 & 0.0003 \\
4 & 1 & 4 & 3 & 0 & 3 & 5223.0530 & 0.0047 \\
5 & 1 & 5 & 4 & 0 & 4 & 6219.3117 & 0.0177 \\
6 & 0 & 6 & 5 & 1 & 5 & 5946.3597 & -0.0015 \\
2 & 2 & 1 & 1 & 1 & 0 & 5257.7327 & -0.0035 \\
3 & 2 & 2 & 2 & 1 & 1 & 6320.9957 & 0.0030 \\
3 & 2 & 1 & 2 & 1 & 2 & 6488.5886 & 0.0066 \\
4 & 2 & 3 & 3 & 1 & 2 & 7357.6964 & -0.0081 \\
4 & 2 & 2 & 3 & 1 & 3 & 7702.8236 & 0.0024 \\
3 & 2 & 1 & 2 & 1 & 2 & 6488.5886 & 0.0066 \\
2 & 1 & 1 & 1 & 0 & 1 & 3327.7598 & 0.0098 \\
3 & 1 & 2 & 2 & 0 & 2 & 4522.9746 & 0.0036 \\
4 & 1 & 3 & 3 & 0 & 3 & 5747.5686 & -0.0069 \\
2 & 2 & 0 & 1 & 1 & 0 & 5259.7542 & -0.0117 \\
2 & 2 & 1 & 1 & 1 & 1 & 5310.2281 & 0.0048 \\
3 & 2 & 1 & 2 & 1 & 1 & 6331.1232 & 0.0023 \\
3 & 2 & 2 & 2 & 1 & 2 & 6478.4490 & -0.0049 \\
4 & 2 & 2 & 3 & 1 & 2 & 7387.9336 & -0.0144 \\
4 & 2 & 3 & 3 & 1 & 3 & 7672.5811 & 0.0033 \\
4 & 0 & 4 & 3 & 1 & 2 & 3259.1516 & -0.0057 \\
5 & 0 & 5 & 4 & 1 & 3 & 4234.4215 & -0.0061
\end{tabular}

Table S50. Measured frequencies ( $v_{\text {obs }}$ ) and residuals ( $\nu_{\text {obs }}-v_{\text {cal }}$ ) of the rotational transitions of the parent species of isomer $\mathbf{A X a - 2} \alpha$ of limonene- $\mathrm{H}_{2} \mathrm{O}$.

\begin{tabular}{cccccccc}
\hline$J^{\prime}$ & $K_{-1}^{\prime}$ & $K^{\prime}{ }_{+1}$ & $J^{\prime \prime}$ & $K^{\prime \prime}{ }_{-1} K^{\prime \prime}{ }_{+1}$ & $v_{\text {obs }} / \mathrm{MHz}$ & \multicolumn{1}{c}{$v_{\text {obs }}-v_{\text {calc }} / \mathrm{MHz}$} \\
\hline 2 & 1 & 2 & 1 & 1 & 1 & 3013.9702 & -0.0019 \\
2 & 0 & 2 & 1 & 0 & 1 & 3137.3701 & 0.0033 \\
2 & 1 & 1 & 1 & 1 & 0 & 3410.6439 & -0.0001 \\
3 & 1 & 3 & 2 & 1 & 2 & 4480.3715 & 0.0051 \\
3 & 0 & 3 & 2 & 0 & 2 & 4569.7781 & -0.0046 \\
4 & 1 & 4 & 3 & 1 & 3 & 5918.8697 & 0.0039 \\
4 & 0 & 4 & 3 & 0 & 3 & 5960.3787 & -0.0080 \\
4 & 1 & 3 & 3 & 1 & 2 & 6609.3288 & 0.0114 \\
5 & 1 & 5 & 4 & 1 & 4 & 7339.6340 & 0.0050 \\
3 & 2 & 2 & 2 & 2 & 1 & 4818.4306 & -0.0080 \\
3 & 2 & 1 & 2 & 2 & 0 & 5067.1126 & 0.0001 \\
4 & 2 & 2 & 3 & 2 & 1 & 6824.2999 & -0.0062 \\
5 & 2 & 4 & 4 & 2 & 3 & 7866.2221 & -0.0078 \\
4 & 3 & 2 & 3 & 3 & 1 & 6533.5854 & 0.0023 \\
4 & 3 & 1 & 3 & 3 & 0 & 6636.4802 & 0.0049
\end{tabular}

Table S51. Measured frequencies ( $\left.v_{\text {obs }}\right)$ and residuals ( $v_{\text {obs }}-v_{\text {cal }}$ ) of the rotational transitions of the DOH isotopic species of isomer AXa-2 $\alpha$ of limonene- $\mathrm{H}_{2} \mathrm{O}$.

\begin{tabular}{cccccccc}
\hline$J^{\prime}$ & $K_{-1}^{\prime}$ & $K^{\prime}{ }_{+1} J^{\prime \prime}$ & $K^{\prime \prime}{ }_{-1} K^{\prime \prime}{ }_{+1}$ & $v_{\text {obs }} / \mathrm{MHz}$ & \multicolumn{1}{c}{$v_{\text {obs }}-v_{\text {calc }} / \mathrm{MHz}$} \\
\hline 2 & 1 & 2 & 1 & 1 & 1 & 3005.3630 & -0.0102 \\
2 & 0 & 2 & 1 & 0 & 1 & 3127.4767 & -0.0021 \\
2 & 1 & 1 & 1 & 1 & 0 & 3413.5778 & 0.0012 \\
3 & 1 & 3 & 2 & 1 & 2 & 4463.9291 & 0.0008 \\
3 & 0 & 3 & 2 & 0 & 2 & 4547.3062 & -0.0011 \\
3 & 1 & 2 & 2 & 1 & 1 & 5053.5588 & 0.0019 \\
4 & 1 & 4 & 3 & 1 & 3 & 5893.4712 & -0.0004 \\
4 & 0 & 4 & 3 & 0 & 3 & 5929.5293 & 0.0008 \\
4 & 1 & 3 & 3 & 1 & 2 & 6591.0679 & 0.0000 \\
5 & 1 & 5 & 4 & 1 & 4 & 7305.5863 & 0.0089 \\
5 & 0 & 5 & 4 & 0 & 4 & 7317.6568 & -0.0057 \\
3 & 2 & 2 & 2 & 2 & 1 & 4814.2078 & 0.0181 \\
3 & 2 & 1 & 2 & 2 & 0 & 5081.0851 & -0.0046 \\
4 & 2 & 3 & 3 & 2 & 2 & 6352.5081 & -0.0071 \\
5 & 2 & 4 & 4 & 2 & 3 & 7843.9754 & -0.0021 \\
4 & 3 & 2 & 3 & 3 & 1 & 6536.1165 & -0.0004
\end{tabular}


Table S52. Measured frequencies ( $\left.v_{\text {obs }}\right)$ and residuals ( $v_{\text {obs }}-v_{\text {cal }}$ ) of the rotational transitions of the DOD isotopic species of isomer AXa-2 $\alpha$ of limonene- $\mathrm{H}_{2} \mathrm{O}$.

\begin{tabular}{cccccccc}
\hline$J^{\prime}$ & $K_{-1}^{\prime}$ & $K^{\prime}{ }_{+1}$ & $J^{\prime \prime}$ & $K^{\prime \prime}{ }_{-1} K^{\prime \prime}{ }_{+1}$ & $v_{\text {obs }} / \mathrm{MHz}$ & $v_{\text {obs }} v_{\text {calc }} / \mathrm{MHz}$ \\
\hline 3 & 1 & 3 & 2 & 1 & 2 & 4399.5296 & -0.0152 \\
3 & 0 & 3 & 2 & 0 & 2 & 4470.6676 & -0.0188 \\
4 & 1 & 4 & 3 & 1 & 3 & 5800.7374 & -0.0025 \\
4 & 0 & 4 & 3 & 0 & 3 & 5827.3017 & 0.0077 \\
4 & 1 & 3 & 3 & 1 & 2 & 6507.7760 & 0.0013 \\
5 & 1 & 5 & 4 & 1 & 4 & 7185.4967 & 0.0096 \\
5 & 1 & 4 & 4 & 1 & 3 & 7879.8819 & 0.0011 \\
3 & 2 & 2 & 2 & 2 & 1 & 4774.5449 & 0.0122 \\
3 & 2 & 1 & 2 & 2 & 0 & 5078.3925 & -0.0046
\end{tabular}

Table S53. Measured frequencies ( $v_{\text {obs }}$ ) and residuals ( $v_{\text {obs }}-v_{\text {cal }}$ ) of the rotational transitions of the $\mathrm{H}_{2}{ }^{18} \mathrm{O}$ isotopic species of isomer $\mathbf{A X a - 2} \alpha$ of limonene- $\mathrm{H}_{2} \mathrm{O}$.

\begin{tabular}{cccccccc}
\hline$J^{\prime}$ & $K_{-1}^{\prime}$ & $K^{\prime}{ }_{+1}$ & $J^{\prime \prime}$ & $K^{\prime \prime}{ }_{-1} K^{\prime \prime}{ }_{+1}$ & $v_{\text {obs }} / \mathrm{MHz}$ & $v_{\text {obs }}-v_{\text {calc }} / \mathrm{MHz}$ \\
\hline 4 & 1 & 4 & 3 & 1 & 3 & 5786.9640 & 0.0139 \\
4 & 0 & 4 & 3 & 0 & 3 & 5815.6910 & -0.0044 \\
5 & 1 & 5 & 4 & 1 & 4 & 7169.0909 & -0.0015 \\
3 & 1 & 3 & 2 & 1 & 2 & 4388.0367 & -0.0060 \\
2 & 1 & 1 & 1 & 1 & 0 & 3384.9435 & 0.0019 \\
3 & 1 & 2 & 2 & 1 & 1 & 4998.7533 & 0.0049 \\
3 & 2 & 2 & 2 & 2 & 1 & 4757.6436 & -0.0019 \\
4 & 2 & 3 & 3 & 2 & 2 & 6267.1734 & -0.0064
\end{tabular}

